Spin-polarized high-energy scattering of charged leptons on nucleons

This article has been downloaded from IOPscience. Please scroll down to see the full text article.

2010 Rep. Prog. Phys. 73016201

(http://iopscience.iop.org/0034-4885/73/1/016201)

View the table of contents for this issue, or go to the journal homepage for more

Download details:

IP Address: 141.34.3.101

The article was downloaded on $24 / 03 / 2011$ at $14: 13$

Please note that terms and conditions apply. 


\title{
Spin-polarized high-energy scattering of charged leptons on nucleons
}

\author{
M Burkardt ${ }^{1,4}$, C A Miller ${ }^{2}$ and W-D Nowak ${ }^{3}$ \\ ${ }^{1}$ Department of Physics, New Mexico State University, Las Cruces, NM 88003-0001, USA \\ 2 TRIUMF, Vancouver, British Columbia V6T 2A3, Canada \\ ${ }^{3}$ DESY, 15738 Zeuthen, Germany \\ E-mail: burkardt@nmsu.edu,miller@triumf.ca and Wolf-Dieter.Nowak@desy.de
}

Received 3 June 2009

Published 23 December 2009

Online at stacks.iop.org/RoPP/73/016201

\begin{abstract}
The proton is a composite object with spin one-half, understood to contain highly relativistic spin one-half quarks exchanging spin-one gluons, each possibly with significant orbital angular momenta. While their fundamental interactions are well described by quantum chromodynamics (QCD), our standard theory of the strong interaction, non-perturbative calculations of the internal structure of the proton based directly on QCD are beginning to provide reliable results. Most of our present knowledge of the structure of the proton is based on experimental measurements interpreted within the rich framework of QCD. An area presently attracting intense interest, both experimental and theoretical, is the relationship between the spin of the proton and the spins and orbital angular momenta of its constituents. While remarkable progress has been made, especially in the last decade, the discovery and investigation of new concepts have revealed that much more remains to be learned. This progress is reviewed and an outlook for the future is offered.
\end{abstract}

(Some figures in this article are in colour only in the electronic version)

This article was invited by Professor P Soding.

\section{Contents}

1. Introduction

2. The longitudinal spin structure of the nucleon

2.1. Introduction

2.2. Axial charge of the nucleon

2.3. Inclusive DIS experiments

2.4. Quark helicity densities from inclusive DIS data 8

2.5. Helicity densities of sea quarks from semiinclusive DIS data

2.6. Helicity densities from a global analysis in NLO 12

2.7. Comparison of first moments of helicity densities 14

2.8. Gluon helicity distribution 14

3. The transverse spin structure of the nucleon 16

3.1. Transverse spin in DIS

3.2. Tensor charge of the nucleon

3.3. Transversity in the Drell-Yan (DY) reaction

3.4. Intrinsic parton transverse momentum

8

4. Towards a three-dimensional picture of the nucleon

4.1. Generalized parton distributions 26

4.2. General properties of GPDs 28

4.3. Impact-parameter-dependent parton distributions

4.4. QCD simulations on a space-time lattice

4.5. Access to quark GPDs through exclusive lepton-nucleon scattering

4.6. GPD parametrizations

4.7. DVCS experimental results

4.8. Quark total angular momenta

${ }^{4}$ Present address: Thomas Jefferson National Accelerator Facility, Newport News, VA 23606, USA. 


\section{The spin budget of the nucleon}

5.1. Decomposing the nucleon spin

5.2. Implications for quark angular momenta

\section{Conclusions and outlook Acknowledgments References}

\section{Introduction}

The nucleon (proton or neutron) is a suitable object for our curiosity in view of the fact that it accounts for most of the directly visible mass of the universe. Half a century ago, elastic electron-proton scattering provided direct experimental evidence [1] that the proton has a finite size of about $1 \mathrm{fm}$. (With the benefit of hindsight, we now realize that its alreadyknown large anomalous magnetic moment could already have been interpreted to indicate that it is not a point-like object [2].) The amazing variety of baryons and mesons discovered using new multi-GeV proton accelerators in the 1950s and 1960s demanded some explanation in terms of an underlying structure that would unify their observed quantum numbers and decay modes. Such a composition in terms of more elementary fractionally charged spin $-\frac{1}{2}$ particles called 'quarks' was postulated in 1964 [3,4]. Then the internal structure of the proton came under direct experimental observation in the late 1960s at the SLAC laboratory, where an intense electron beam became available with an energy large compared with the relevant scale of $\sim 1 \mathrm{GeV}$. In these now-famous deepinelastic scattering (DIS) experiments, substantial amounts of energy and momentum are transferred to the proton target, causing it to disintegrate. In 1969, it was hypothesized that the energy and momentum were being transferred to internal constituents of the proton, called 'partons' [5]. Information about the properties of the constituents can be inferred from the distribution of the scattering cross section in these measured transfers. In the same exciting year, it was realized that the data showed a property called 'scaling' [6]: at large enough momentum transfer, the kinematic dependence of the cross section resembles that for elastic scattering off point-like charged particles that might be identified with those partons. As the properties of the cross section also indicated that the partons have spin $\frac{1}{2}$, it became accepted that the charged partons could be assumed to be the hypothesized quarks. However, it emerged that the total momentum carried by the observed quarks accounts for only about half of the proton's momentum. This suggested that there must also be electrically neutral partons, possibly being exchanged by quarks to provide their binding force, leading to the name 'gluons'. Then in the early 1970s, an elegant field theory was devised to explain this binding, as well as the observed weakening of this force at short distances or large momentum transfers, known as 'asymptotic freedom' [7,8]. This theory became known as quantum chromodynamics (QCD), the contemporary standard theory of strong interactions.

The QCD Lagrangian can be written in one line. Nevertheless, as in other fields of science, simple rules can give rise to complex behaviour. The unique richness of this field theory accounts for the challenge presented by the study of hadron structure. The QCD vacuum is believed to resemble a 'dual superconductor', which, unlike conventional
QED superconductors, repels colour electric fields rather than magnetic fields. Unlike in QED, where vacuum polarization screens charges as their separation increases, the $Q C D$ vacuum provides anti-screening that results in a rapidly increasing ('running') QCD coupling constant $\alpha_{s}$ at larger distance scales or smaller momentum transfer. Moreover, the analogue of the dual Meissner effect in a superconductor leads in the QCD vacuum to the formation of a colour flux tube connecting quarks and antiquarks in the same way as the magnetic flux connecting two magnetic monopoles in a conventional (QED) superconductor would be squeezed into narrow flux tubes. This apparently leads to the phenomenon of confinement, as free quarks are not observed, although no proof has been found for the theorem that confinement inevitably follows from QCD. In any case, the quantitative prediction of hadron properties in QCD is presently subject to uncontrolled approximations. Laborious simulations on a Euclidian lattice are now producing such predictions for an increasing number of observables. In contrast, the asymptotic freedom of QCD at short distances allows precise perturbative treatment of hard interactions. Hence such hard interactions with bound partons can be used to experimentally probe the structure of hadrons. A good representative for the energies that are required to reach the perturbative regime is provided by the scale parameter $\Lambda_{\mathrm{QCD}}$. While this parameter appears also in the description of the running of the QCD coupling, it provides a rough estimate about the scale of many non-perturbative effects, such as the energy density needed to break the superconducting phase. The numerical value of $\Lambda_{\mathrm{QCD}}$ depends somewhat on the renormalization procedure (scheme) that is used in its definition, but a value $\Lambda_{\mathrm{QCD}} \approx 200-300 \mathrm{MeV}$ provides a good scale for estimates of the relevance of effects governed by the non-trivial $Q C D$ vacuum structure.

Quark kinetic energy together with QCD field energy accounts for most of the mass of the nucleon, which in turn accounts for most of the mass of the visible universe. Hence the nucleon is a worthy subject of investigation. DIS of leptons has continued to provide most of our present knowledge of nucleon structure. As shown in figure 1, a virtual photon $\gamma^{*}$ emitted by the incident lepton e and absorbed by a bound quark of flavour up $(u)$ or down $(d)$ in the nucleon $\mathrm{N}$ has space-like fourmomentum $q$ that can be inferred in inclusive measurements in which only the scattered lepton $\mathrm{e}^{\prime}$ is detected. The motion of the mostly low-mass $u$ and $d$ flavours of quarks populating the proton is highly relativistic. Hence virtual quark-antiquark pairs are abundant and may be manifest in suitable processes. (In the general context of DIS, the word 'quarks' can be taken to also include antiquarks.) The relativistic motion also implies that DIS results are best interpreted in a reference frame where the target proton has 'infinite' momentum opposite to that of the incident virtual photon. One may intuitively think of this tactic as freezing the transverse motion of the partons during the interaction time through time dilatation 


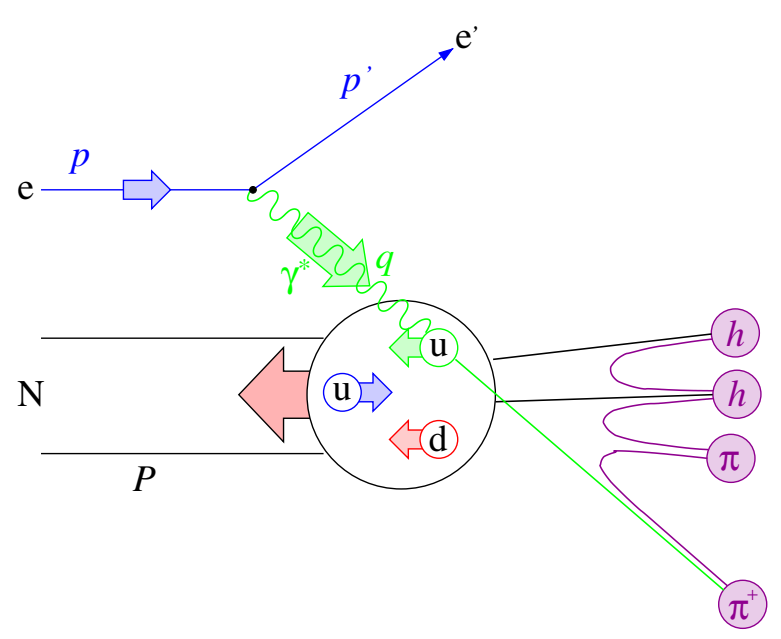

Figure 1. Simplified schematic diagram of semi-inclusive DIS. The large shaded arrows represent typical helicities, as described later in the text.

(while remembering that their intrinsic transverse momentum $p_{\mathrm{T}}$ is of course invariant under such a boost). A virtual photon has adequate spatial resolution to resolve the target's internal structure [9] if its virtuality $Q^{2} \equiv-q^{2}$ is sufficiently large, a requirement empirically found to be at least $Q^{2}>1 \mathrm{GeV}^{2}$. Given this condition, a key experimental observable is the distribution of cross sections in the Bjorken scaling variable $x_{\mathrm{B}} \equiv Q^{2} /(2 P \cdot q)$. In the infinite-momentum frame, $x_{\mathrm{B}}$ can be interpreted as the fraction $x$ of the proton's four-momentum $P$ that was carried by the quark before it was struck by the virtual photon. Thus we distinguish here between the experimentally obervable kinematic variable $x_{\mathrm{B}}$ and the theoretical concept of $x$ as a parton momentum fraction. Only in the parton-model approximation to the DIS process can they be equated. In this framework, the dependence of the DIS cross section on $x_{\mathrm{B}}$ provides information about the $x$-distribution of partons in the target (often called parton distribution functions or PDFs). For a hypothetical hadron consisting of three non-relativistic quarks with the same large mass, this distribution would be a $\delta$-function at $x=\frac{1}{3}$. For systems of light quarks, this is far from being the case.

PDFs can be considered to be intrinsic properties of hadrons only if they are 'universal', i.e. if they can be applied in descriptions of various hard processes involving the same hadron. Asymptotic freedom of QCD has provided the means to overcome its complicating aspects to prove profound 'factorization theorems' showing that cross sections for several experimentally important hard processes can be represented as a product of a cross section for one or more perturbatively calculable partonic subprocesses and one or more non-perturbative but universal parton distributions. For the case of a DIS cross section $\sigma\left(x_{\mathrm{B}}, Q^{2}\right)$ in the Bjorken limit $Q^{2} \rightarrow \infty$ at fixed $x_{\mathrm{B}}$, the quark distribution $q_{f}\left(x_{\mathrm{B}}, Q^{2}\right)$ appears in a sum over quark and antiquark flavours $f=$ $u, d, s, \bar{u}, \bar{d}, \bar{s} \ldots$ weighted by the squares of their electric charges $e_{f}$ in units of the elementary charge:

$$
\sigma\left(x_{\mathrm{B}}, Q^{2}\right) \propto \sum_{f} e_{f}^{2} q_{f}\left(x_{\mathrm{B}}, Q^{2}\right)
$$

A key feature here is the incoherence between the amplitudes for the various quark flavours. At least in the Bjorken limit, this may be understood to be associated with the possible distinguishability of hadronic final states arising from different quark flavours, so that interference cannot arise. Parton distributions such as $q_{f}\left(x, Q^{2}\right)$ have probabilistic interpretations as parton number densities. At finite values of $Q^{2}$, the relationship between PDFs and observable cross sections is only a fairly crude approximation at leading order in $\alpha_{s}$ as in (1), so the perturbative expansion is continued to next-to-leading-order (NLO) or even further. Based on the extensive available data set for DIS as well as other processes, there is now available a precise (except at very small or large $x$ ) parametrization of $x$ distributions of the $u$ and $d$ 'valence' quarks that determine the quantum numbers of the proton, and less precisely of the virtual 'sea' quarks that can be produced by gluon splitting, such as anti-up $(\bar{u}), \bar{d}$ and strange $(s, \bar{s})$.

The dependence of parton distributions on the 'hard scale' $Q^{2}$ of the process is weak (logarithmic), and can be understood to arise from the finer spatial resolution of a probe at a harder scale, which allows it to resolve more of the virtual partons that 'dress' each quark. These virtual $q \bar{q}$ or gluon pairs are abundant because their small or zero mass makes them energetically inexpensive. With quantum fluctuations so important at short distances, the 'appearance' of the nucleon depends on how it is probed. This might be considered to be analogous to the familiar change in appearance of a macroscopic object when viewed in light of different colours or wavelengths, due in part to the interactions between the atoms of its surface. Unlike the $x$ dependence, the $Q^{2}$ dependence of parton distributions is perturbatively calculable in QCD at large enough $Q^{2}$. Thus a distribution that is known over the range $x_{0}<x<1$ (or alternatively $0<x<x_{0}$ ) for one value of $Q^{2}$ can be 'evolved' to the distribution over the same $x$-range at a larger (smaller) value of $Q^{2}$, using the so-called DGLAP integro-differential equations [10-12]. The evolution kernels for several parton distributions have been computed up to nextto-next-to-leading-order (NNLO) in $\alpha_{s}$ [13-16], meaning that participation of gluons in hard QCD interactions is taken into account. Gluons may be created in these hard interactions, or they may be found in the target nucleon. Hence, at order $\alpha_{s}$ or higher, the DGLAP equations couple the distributions for quarks and gluons. This feature has been exploited in global fits of all available inclusive DIS data, which cover a range in $Q^{2}$ from 1 to $50 \mathrm{GeV}^{2}$, to extract information about the gluon distribution $\mathrm{G}(x)$. From this and other types of data, $\mathrm{G}(x)$ is now quite well determined except at small $x$ with moderately small $Q^{2}$. For convenience in the rest of this paper, we suppress the $Q^{2}$ dependence of distributions in our notation.

Parton distributions depend on the order of perturbation theory relating them to experimental observables. Beyond leading order in $\alpha_{s}$, they lose their probabilistic interpretation as number densities, and can even become negative. However, at each order, they remain universal among various observable processes in which they participate, and hence are still much more than just parametrizations. Furthermore, their basic construction is motivated by simple semi-classical partonmodel ideas, but implemented to account for the intricacies of a quantum system. 


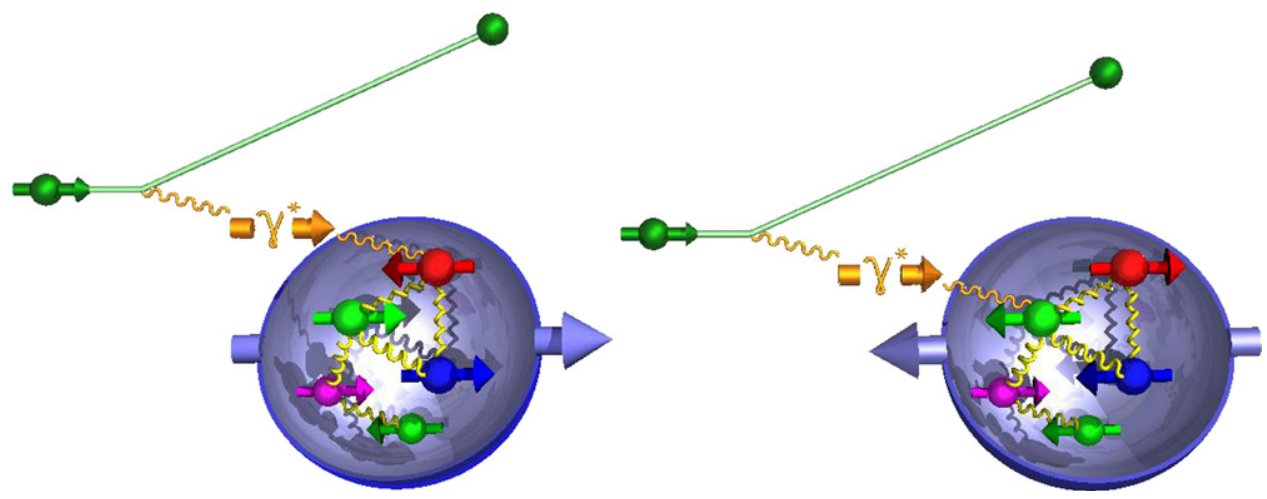

Figure 2. Visualization of scattering of longitudinally polarized leptons and protons.

For values of $Q^{2}$ below $\sim 10 \mathrm{GeV}^{2}$, there may be additional contributions to the cross section that scale as $1 / Q^{n}, n>0$. These may be corrections arising because the mass of the target nucleon is not negligible compared with $Q^{2}$, or they may be due to 'higher twist' subprocesses, in which an additional parton in the target nucleon experiences a hard interaction. (Some authors apply the term 'higher twist' for all 'power-suppressed' terms, even those suppressed by factors of $1 / Q^{n}$ arising only because of kinematic relationships. In this case, contributions involving an additional parton are designated as 'interaction dependent' or 'dynamical higher twist'.) For DIS processes, leading twist is twist-2, with no such contributions.

Baryons are the fermionic bound states of the spin- $\frac{1}{2}$ quarks. All known baryons have quantum numbers consistent with those of a set of three quarks of particular flavours. (Bound states of 5 or more quarks are theoretically possible, and in fact such wave function components may contribute to the ground states of ordinary baryons such as the proton.) Soon after the conception of quarks as the constituents of the nucleon, non-relativistic models were constructed in which the mass of the nucleon was directly attributed to three massive quarks. One of the successes of these models was considered to be the remarkable consistency of the measured magnetic moments of many baryons with a combination of Dirac magnetic moments of their three 'constituent quarks'.

Then in the 1970s, the internal spin structure of the proton came under investigation via DIS of helicity-polarized lepton beams on hydrogenous targets containing protons polarized with the beam axis as quantization axis, i.e. longitudinal polarization. The exchanged virtual photon inherits the helicity of the beam lepton, to a degree that depends on the lepton kinematics. Due to conservation of helicities in hard or short-distance interactions, only quarks with the opposite spin direction (i.e. same helicity) as the spin-1 photon can absorb it in the leading-order process $\gamma^{*} q \rightarrow q$ (see figure 2). Hence the 'polarized virtual-photon beam' selects quarks of one helicity. From the difference in cross sections with the same or opposite polarizations of the beam and the target, it is therefore possible to extract the number densities of quarks having the same $(q \overrightarrow{\vec{f}})$ or opposite $(q \underset{f}{\vec{f}})$ helicity as the target proton in the infinite-momentum frame. Here the symbol $\rightarrow(\Rightarrow)$ designates the helicity of the quark (target proton). The quark helicity distribution $\Delta q_{f}(x)$ is then defined as $\Delta q_{f}(x)=q_{\vec{f}}(x)-$ $q_{f}^{\vec{\epsilon}}(x)$, while the polarization-averaged distribution $q_{f}(x)$ introduced above can be written $q_{f}(x)=q_{\vec{f}}^{\overrightarrow{\vec{f}}}(x)+q_{f}^{\vec{\rightleftarrows}}(x)$. From these definitions, the obvious 'positivity limit' arises: $\left|\Delta q_{f}(x)\right|<q_{f}(x)$. The distribution $q_{f}(x)\left[\Delta q_{f}(x)\right]$ is sometimes written as $f_{1}^{q}(x)\left[g_{1}^{q}(x)\right]$, although this is arguably confusing, because the spin structure functions $f_{1}$ and $g_{1}$ are defined to be functions of the observable kinematic quantity $x_{\mathrm{B}}$, while the parton distributions are defined to be functions of the parton momentum fraction $x$, with the relations between them being subject to approximations such as single-photon exchange and the quark-parton model. For convenience hereafter, we use the term '(un)polarized' to describe measurements, cross sections or parton distributions, meaning (polarization averaged) polarization dependent. Polarized PDFs evolve logarithmically with $Q^{2}$ according to a set of DGLAP equations that differs somewhat from those for the unpolarized case.

Certain $x$-moments of parton distributions (integrals over $x$ weighted with $x^{n}$ ) have important physical interpretations. First $(n=0)$ moments can be conveniently written as, e.g., $\Delta q_{f} \equiv \int \mathrm{d} x \Delta q_{f}(x)$. A quantity that has played a central role in the discussion of nucleon spin structure in recent decades is the flavour sum $\Delta \Sigma \equiv \sum_{f} \Delta q_{f}$, as it can be interpreted as the net contribution of the quark helicities to the spin of the nucleon. (At the present precision of the field, the proton and neutron can be considered to be related by isospin symmetry.) Note that contributions of individual quark flavours may tend to cancel each other in this sum. A pivotal event in this field in the late 1980s was the DIS measurement with longitudinal polarization of both muon beam and hydrogenous target by the EMC experiment $[17,18]$ at the CERN laboratory, which for the first time extended over a wide enough range in $x_{\mathrm{B}}$ that $\Delta \Sigma$ could be inferred, albeit under the assumption of SU(3) flavour symmetry (neglecting the effects of the $\sim 100 \mathrm{MeV}$ mass of the strange quark), which allows use of additional constraints from measured weak decay lifetimes of hyperons. The surprisingly small resulting value suggested that quark helicities make little net contribution to the nucleon spin, in contrast to naive expectations based on the baryon magnetic moments mentioned above. In language that with the benefit of hindsight may now seem extravagant, this finding was 

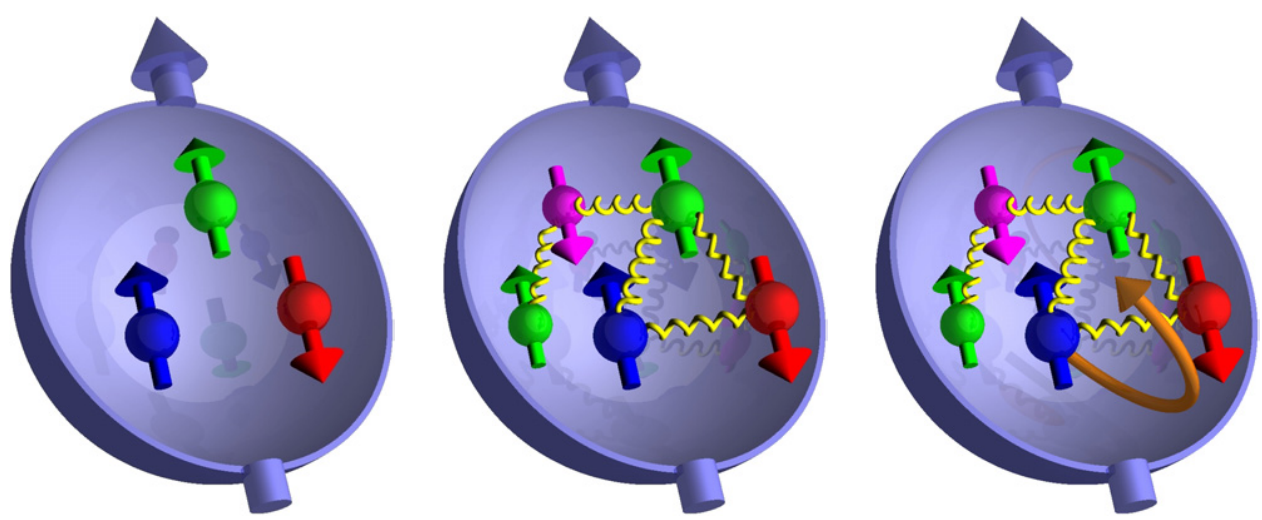

Figure 3. Illustration of nucleon structure. Left: valence quarks only. Middle: gluons and quark-antiquarks in addition. Right: orbital angular momentum of quarks.

called a 'spin crisis', and inspired more than a thousand theoretical papers attempting its interpretation, and several new experiments with the aim of confirming the measurement with higher precision, and extending it to include the 'neutron' target.

Because of the relativistic nature of bound systems of light quarks, there exists a third independent flavour set of quark distributions that have a probabilistic interpretation as number densities. These quark transversity distributions $\delta q_{f}(x)$ are needed to describe the transverse ( $\left.\uparrow\right)$ polarization of quarks in a nucleon polarized transversely $(\Uparrow)$ with respect to its infinite-momentum: $\delta q_{f}(x) \equiv q_{f}^{\uparrow \Uparrow}(x)-q_{f}^{\uparrow \Downarrow}(x)$. Their probabilistic interpretation applies only in a basis of transverse spin eigenstates, not in the helicity basis relevant for the $\Delta q_{f}(x)$. The distribution $\delta q_{f}(x)$ is sometimes written as $h_{1}^{q}(x)$, although this might be misleading because no spin structure function $h_{1}\left(x_{\mathrm{B}}\right)$ is defined. There exists little experimental information about transversity, because the coupling of hard vector bosons to quarks is insensitive to the transverse polarization of the quark, unless the transverse polarization of the struck quark in the final state is 'measured'. However, some traces of this transverse polarization appear in the structure of the 'jet' of final-state particles produced by the energetic struck quark. Information about transversity has begun to emerge from semi-inclusive measurements with transverse target polarization, which include the detection of one or more hadrons in addition to the scattered lepton (see figure 1).

All parton distributions actually have a two-dimensional dependence on both $x$ and the intrinsic quark momentum component $p_{\mathrm{T}}$ transverse to the nucleon's 'infinite' momentum. This two-dimensional dependence does not factorize. The three flavour sets of distributions mentioned above that have probabilistic interpretations are considered basic in the sense that they survive integration over $p_{\mathrm{T}}$, and it is this integral that is signified when only the $x$ argument appears. Five other sets of distributions have been identified that also have probabilistic interpretations but do not survive such integration. Some of these have been found to be particularly interesting, as in DIS they embody the effects of a 'final-state interaction (FSI)' between the departing struck quark and the rest of the target nucleon. The contribution of such a distribution to the cross section depends on polarization and momentum vectors in a manner that is called 'T-odd', although this does not refer to fundamental time symmetry. The study of these T-odd effects has provoked a reconsideration of the meaning of parton distributions and their universality. Experimental evidence for T-odd effects has now been observed in the same semi-inclusive data from which information about transversity has been extracted.

The dependence of the elastic lepton-nucleon cross section on the momentum transfer is represented by the elastic 'form factor', as its Fourier transform can be interpreted as the spatial charge distribution in the nucleon. Up until about a decade ago, information about hadron structure embodied in elastic form factors and in PDFs was interpreted separately. It was then discovered that these concepts could be unified in what became known as generalized parton distributions (GPDs), also called off-forward or skewed parton distributions. Even more profound was the finding that the dependence of these GPDs on three kinematic variables encodes much more detailed information, about e.g. parton orbital angular momentum [19] and correlations between transverse position and longitudinal momentum fraction of partons [20-23]. Of particular interest is that a second moment of a certain combination of GPDs for each parton flavour was found to represent the total (helicity plus orbital) contribution of that flavour to the spin of the nucleon [19]. This offers the first opportunity for experimental access to parton orbital angular momentum, as it was discovered that the additional information could, in principle, be obtained from experimental studies of hard exclusive processes involving additional hard interaction(s), yet 'replacing' the struck quark in the target nucleon to leave it intact. Most prominent among these processes is deeply virtual Compton scattering (DVCS), the hard exclusive production of an energetic real photon. Within the last decade, rapid theoretical progress has been made in understanding this new subject, and several DVCS observables have been measured.

Thus the evolution of our understanding of nucleon spin structure might be imaginatively summarized by figure 3. 


\section{The longitudinal spin structure of the nucleon}

\subsection{Introduction}

As mentioned above, parton distributions have been investigated experimentally using DIS of lepton beams. In order to measure helicity distributions $\Delta q_{f}(x)$, both beam and target must be longitudinally polarized. The relevant observable is the difference in cross sections with the same or opposite polarizations of the beam and the target:

$$
\sigma_{\mathrm{LL}}\left(x_{\mathrm{B}}\right) \equiv \frac{1}{2}\left[\sigma^{\vec{\Rightarrow}}\left(x_{\mathrm{B}}\right)-\sigma^{\vec{\rightleftarrows}}\left(x_{\mathrm{B}}\right)\right] \text {. }
$$

However, measurements of absolute cross sections are technically difficult with polarized targets. Hence the experimental results are typically extracted from the data as 'double-spin' asymmetries:

$$
A_{\|}\left(x_{\mathrm{B}}\right) \equiv \frac{\sigma_{\mathrm{LL}}\left(x_{\mathrm{B}}\right)}{\sigma_{\mathrm{UU}}\left(x_{\mathrm{B}}\right)}
$$

where $\mathrm{L}(\mathrm{U})$ designates longitudinal polarization (unpolarized) appearing in pairs corresponding to the beam followed by the target. The polarization-averaged cross section

$$
\sigma_{\mathrm{UU}}\left(x_{\mathrm{B}}\right) \equiv \frac{1}{2}\left[\sigma^{\vec{\Rightarrow}}\left(x_{\mathrm{B}}\right)+\sigma^{\vec{k}}\left(x_{\mathrm{B}}\right)\right]
$$

has been previously measured precisely using unpolarized targets. Using these known values and measured values of $A_{\|}, \sigma_{\mathrm{LL}}$ can be extracted. This approach based on cross sections avoids confusions that sometimes arise. For example, it has been proposed that if the values of $\sigma_{\mathrm{LL}}$ are interpreted theoretically in leading order, then the values of $\sigma_{\mathrm{UU}}$ should be calculated in leading order from a theoretical representation in terms of parton distributions, neglecting the portion involving the so-called 'longitudinal' virtual photons!' This misses the key point that $\sigma_{\mathrm{UU}}$ became involved only because of the experimental convenience of asymmetry measurements, and is irrelevant to the theoretical interpretation of spin observables. Hence it should be treated empirically in terms of experimental data, with no influence from theoretical concepts such as PDFs or order in $\alpha_{s}$. Another point of confusion is that the extraction has often been formulated in a way influenced by the technical convenience that precisely measured values of $\sigma_{\mathrm{UU}}$ are typically represented in terms of an unpolarized structure function called $F_{2}$ and the ratio $R$ of the cross sections for longitudinal and transverse photons. Unfortunately, various values $R$ were used to extract the values of $F_{2}$, so the world data set for $F_{2}$ contains inconsistencies. This confusion can be avoided by using in equation (3) the original experimental values of $\sigma_{\mathrm{UU}}$, reconstructed if necessary using the values of $R$ employed in the original extraction of $F_{2}$.

If the measurement of $A_{\|}$is inclusive, i.e. only the scattered lepton is detected while ignoring the produced

\footnotetext{
5 Longitudinal virtual photons are those having 'magnetic' quantum number $m=0$ with respect to the quantization axis along the photon momentum direction, while photons with 'magnetic' quantum number $m= \pm 1$ are called 'transverse'. Real photons are always transverse. Sometimes the word 'polarization' is included in this unfortunate nomenclature, even though the distinction has nothing to do with the longitudinal or transverse direction of a spin quantization axis.
}

hadrons, $\sigma_{\mathrm{LL}}$ is linearly related via a kinematic factor that includes the QED hard scattering cross section to the spin structure function $g_{1}\left(x_{\mathrm{B}}\right)$. (There is a small contribution to $\sigma_{\mathrm{LL}}$ from another structure function $g_{2}$, which has no probabilistic interpretation, and is measured using a transversely polarized target.) At leading order in $\alpha_{s}, g_{1}$ for the proton (p) or neutron (n) the target has a simple probabilistic interpretation in terms of quark helicity densities for flavour $f$ :

$g_{1}^{\mathrm{p}, \mathrm{n}}\left(x_{\mathrm{B}}\right)=\frac{1}{2} \sum_{f} e_{f}^{2}\left(\Delta q_{f}^{\mathrm{p}, \mathrm{n}}\left(x_{\mathrm{B}}\right)+\Delta \bar{q}_{f}^{\mathrm{p}, \mathrm{n}}\left(x_{\mathrm{B}}\right)\right)$.

The same linear combination relates the first moments $\Delta q_{f}$ of the helicity densities to the moments $\Gamma_{1}^{\mathrm{p}, \mathrm{n}}$ of $g_{1}$ :

$\Gamma_{1}^{\mathrm{p}, \mathrm{n}} \equiv \int_{0}^{1} \mathrm{~d} x_{\mathrm{B}} g_{1}^{\mathrm{p}, \mathrm{n}}\left(x_{\mathrm{B}}\right)=\frac{1}{2} \sum_{f} e_{f}^{2}\left(\Delta q_{f}^{\mathrm{p}, \mathrm{n}}+\Delta \bar{q}_{f}^{\mathrm{p}, \mathrm{n}}\right)$.

Since the quark charges enter only as squares, inclusive DIS measurements cannot distinguish quarks from antiquarks, and hence can constrain only combinations of $\Delta u+\Delta \bar{u}, \Delta d+\Delta \bar{d}$ and $\Delta s+\Delta \bar{s}$. As mentioned earlier, charge symmetry is a good approximation, implying $\Delta u^{\mathrm{p}}+\Delta \bar{u}^{\mathrm{p}}=\Delta d^{\mathrm{n}}+\Delta \bar{d}^{\mathrm{n}}$ and $\Delta u^{\mathrm{n}}+\Delta \bar{u}^{\mathrm{n}}=\Delta d^{\mathrm{p}}+\Delta \bar{d}^{\mathrm{p}}$ and $\Delta s+\Delta \bar{s}$ is the same in the neutron and proton. (By convention, when the superscript is absent, the proton is implied.) Hence measurements of $\Gamma_{1}^{\mathrm{p}}$ and $\Gamma_{1}^{\mathrm{n}}$ (inferred from data on proton and, e.g., deuteron targets) provide constraints on two linear combinations of these three unknowns.

A third such constraint can be derived from measurements of the lifetimes of weakly decaying hyperons, under the assumption of the SU(3) flavour symmetry among quark distributions that would apply in the limit of no mass difference among quark flavours. This symmetry has been found to be valid at the few per cent level in a study of the relationship between the lifetimes of hyperons. The additional third constraint allows all three unknown moments to be extracted, and also their flavour-singlet combination

$$
\Delta \Sigma \equiv(\Delta u+\Delta \bar{u})+(\Delta d+\Delta \bar{d})+(\Delta s+\Delta \bar{s}) .
$$

As described below, it has been inferred from a series of DIS experiments of ever increasing precision that $\Delta \Sigma=0.2-0.4$ and $\Delta s+\Delta \bar{s}$ is significantly negative. This implies that there must be substantial contributions from quark orbital angular momentum $L^{q}$ and/or gluon total angular momentum $J_{\mathrm{G}}$, in order to complete the 'proton spin budget'. The experimental investigation of these contributions is the subject of the following sections of this paper. Subtle issues about the conceptual basis of spin decompositions of a system of interacting constituents are discussed in detail in section 5.1.

\subsection{Axial charge of the nucleon}

The axial charge $g_{\mathrm{A}}$ of the nucleon is obtained from the forward matrix element of the operator $\bar{\psi} \gamma^{\mu} \gamma^{5} \psi$ in the same way as the familiar (vector) charge $g_{\mathrm{V}}$ is obtained from the forward matrix element of the operator $\bar{\psi} \gamma^{\mu} \psi$. In terms of quark helicity eigenstates, the contribution of each quark flavour $f$ to the axial charge of the nucleon involves the same linear 


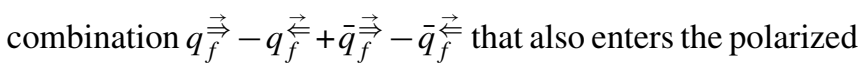
structure function $g_{1}\left(x_{\mathrm{B}}\right)$. As a result, the integral of $g_{1}\left(x_{\mathrm{B}}\right)$ yields the quark-charge-squared-weighted axial charge $g_{\mathrm{A}}$ of the nucleon. Since the isovector axial charge of the nucleon also describes the $\beta$-decay of the neutron, one can thus relate the integral of the isovector polarized structure function $g_{1}\left(x_{\mathrm{B}}\right)$ to the neutron $\beta$-decay constant. In the scaling limit of infinite $Q^{2}$, this relationship between the spin structure functions of the proton and neutron is

$$
\int_{0}^{1} \mathrm{~d} x_{\mathrm{B}}\left[g_{1}^{\mathrm{p}}\left(x_{\mathrm{B}}\right)-g_{1}^{\mathrm{n}}\left(x_{\mathrm{B}}\right)\right]=\frac{1}{6} \frac{g_{\mathrm{A}}}{g_{\mathrm{V}}} .
$$

While we presented here only a simple plausibility argument, this famous Bjorken sum rule (BSR) was derived [24] in the Bjorken limit of infinite $Q^{2}$ even before the advent of QCD, using only Gell-Mann's current algebra relations [25-27] together with a reasonable assumption about smooth asymptotic high-energy behaviour of the virtual Compton amplitude. Within the modern context of QCD where the sum rule can be evolved to finite $Q^{2}$, it can be tested precisely by practical experiments. If a violation were discovered, the implications would be profound. This is expressed eloquently in [28]: 'Occasionally it is worth reminding ourselves what it means to 'understand' something in QCD. In the absence of fundamental understanding we often invoke 'effective descriptions' based on symmetries and low-energy expansions. While they can be extremely useful, we should not forget that a thorough understanding allows us to relate phenomena at very different distance scales to one another. In the case of Bjorken's sum rule, the operator product expansion, renormalization group invariance and isospin conservation combine to relate DIS at high $Q^{2}$ to the neutron's $\beta$-decay axial charge measured at very low energy. Even target mass and higher twist corrections are relatively well understood.'

\subsection{Inclusive DIS experiments}

Experimental information about quark helicity densities in the nucleon has so far come only from DIS of polarized highenergy charged leptons on targets containing polarized protons, or polarized light nuclei containing neutrons. The technologies for polarizing the beam and the target are challenging, and have been the subject of intense efforts over several decades. A high beam polarization of $85 \%$ was achieved in even the first experiments [29], done at SLAC in the 1970s using a $6-23 \mathrm{GeV}$ beam of electrons with polarization inherited in the photo-ionization of lithium atoms selected via the SternGerlach method. However, the beam intensity was quite limited, and this approach was eventually replaced in modern SLAC experiments [30-35] using electron beam energies up to $50 \mathrm{GeV}$, polarized at a level of typically $85 \%$ in the electron source by photoelectric emission from a gallium arsenide surface. The beam intensities increased until they were limited by the heat tolerance of the cryogenic targets. Measurement times are of the order of a few weeks.

Meanwhile, a series of even higher energy experiments at CERN [36-43] used 100-200 GeV beams of muons naturally polarized to a level of $80 \%$ by the weak decay of the precursor $\pi$ or K mesons. These tertiary beams had limited intensities of typically $4.5 \times 10^{7}$ per spill of $2.4 \mathrm{~s}$ duration, with a spill period of $14.4 \mathrm{~s}$, but the more massive muons suffer less radiative energy loss in the target materials than electrons, allowing much larger target thicknesses of the order of metres instead of centimetres. Nevertheless, measurement times are of the order of a year.

Most targets used at SLAC and CERN are cryogenic solids of butanol, ammonia, or more recently ${ }^{6} \mathrm{LiD}$, in a strong magnetic field, with most protons or deuterons in the target polarized through the process of dynamic nuclear polarization (DNP). For DNP to proceed, a suitable material doped with a paramagnetic compound is cooled to temperatures less than about $1 \mathrm{~K}$. In the high magnetic field (2-6.5 T), the unpaired electron of the dopant is polarized to almost $100 \%$, while the proton or deuteron has a polarization of less than $1 \%$. The process of DNP causes most of the electron polarization to be transferred to that of the protons by microwave irradiation of the sample. By this means, proton polarizations of more than $95 \%$ can be achieved. Typical deuteron polarizations are $40-55 \%$; recently though, deuteron polarizations of $80 \%$ have been obtained. However, most of the material encountered by the beam in such solid polarized targets is non-hydrogenous. Hence the asymmetry of interest is strongly diluted by the resulting background events, by a factor of up to about 7 . This constrains the statistical precision that can be achieved, and requires careful background corrections.

As spin structure functions must be determined for both the proton and neutron, and targets of free neutrons are still infeasible, measurements are made using targets containing either deuterons or ${ }^{3} \mathrm{He}$. The structures of these light nuclei are such that the spin of the neutron is strongly correlated with that of the nucleus. Protons can be replaced by deuterons in solidstate targets without fundamental changes in the technology. In contrast, the very different polarized ${ }^{3} \mathrm{He}$ targets consist of a low pressure gas polarized via spin exchange with alkaline atoms, the polarization of which is pumped by an infrared laser. Such targets were used in a pair of experiments at SLAC. The dilution in these targets is contributed by the essentially unpolarized protons in ${ }^{3} \mathrm{He}$ and the thin target windows, and hence is smaller than in the cryogenic solids.

A completely different experimental approach was followed at the DESY laboratory, where the $27.5 \mathrm{GeV}$ HERA storage ring contained beams of typically $40 \mathrm{~mA}$ of electrons or positrons. Such stored high-energy electron beams may become spontaneously polarized via a small polarization asymmetry in the emission of synchrotron radiation by the beam particles as they are deflected by the magnetic fields of the ring [44]. (Several megawatts of power were radiated via synchrotron radiation, and was continuously replenished by a system of accelerating radio-frequency cavities.) The polarization rise time was of order $\frac{1}{2} \mathrm{~h}$, and polarizations as large as $60 \%$ were achieved. To avoid disrupting the stored beam, only very thin targets were possible. These were tenuous gases of nuclear-polarized atoms of hydrogen or deuterium partially contained in an cylindrical open-ended storage cell coaxial with the beam. The polarized atoms were injected into the cell from an atomic beam source based on Stern-Gerlach 
polarization filtering and radio-frequency transitions between atomic substates in a magnetic field. The atoms bounced off the cell walls of order 100 times before escaping from the ends, after which they were differentially pumped away by a large system of turbo-pumps. Polarized hydrogen target thicknesses of about $10^{14}$ nucleons $\mathrm{cm}^{-2}$ were achieved, and there was no target dilution from non-hydrogenous material. Measurement times were of the order of a year.

\subsection{Quark helicity densities from inclusive DIS data}

The data from the first polarized DIS measurements at SLAC were used to test simple theoretical expectations of the time, but did not cover a large enough $x_{\mathrm{B}}$ range to permit the inference of the first moment of $g_{1}$. The first opportunity for this appeared in the late 1980s with the EMC experiment at CERN, which employed $120-200 \mathrm{GeV}$ beams of muons. As already mentioned, the $\Gamma_{1}^{\mathrm{p}}$ moment extracted from this data was interpreted to imply a surprisingly small net contribution of quark helicities to the proton spin, including a negative contribution from the strange sea. The resulting excitement led to a series of experiments of ever-higher precision at SLAC [30,31,33-35], CERN [36-40] and DESY [45-47]. Figure 4 shows published data for the spin structure functions $g_{1}\left(x_{\mathrm{B}}\right)$ of the proton and deuteron, while figure 5 shows the results for the neutron inferred from the data in figure 4, or from measurements with a ${ }^{3} \mathrm{He}$ target. This information is best displayed as $x_{\mathrm{B}} \cdot g_{1}\left(x_{\mathrm{B}}\right)$ versus $\log x_{\mathrm{B}}$, which conserves contributions to the area under the data representing the first moment $\Gamma_{1}$.

A key issue to be addressed using the data sets containing measurements on both proton and 'neutron' targets is the BSR, introduced in section 2.2. To test this rule, the first moments $\Gamma_{1}^{\mathrm{p}}$ and $\Gamma_{1}^{\mathrm{n}}$ must be extracted from the data at some fixed value of $Q^{2}$. However, measurements can never cover the complete range $0<x_{\mathrm{B}}<1$, so the inference of $\Gamma_{1}$ requires some estimate of the contributions to the moments from the unmeasured regions at the extremes of this range. These estimates are based on some assumption or ansatz motivated by reasonable expectations. Another problem is that these data were all produced by fixed-target experiments, resulting in a strong kinematic correlation between $x_{\mathrm{B}}$ and the hard scale $Q^{2}$, so that each data point corresponds to a different value of $Q^{2}$, as shown in the bottom panels of figures 4 and 5. This implies that it is impossible to directly calculate the first moment of $g_{1}\left(x_{\mathrm{B}}\right)$ from experimental data. Hence, some assumption or ansatz is typically employed to approximately 'evolve' the data from that experiment to a common value of $Q^{2}$. (The resulting model uncertainty has not always been estimated and included with the final result.) Applying these approaches to the results of individual experiments has resulted in agreement with the BSR within the experimental uncertainty, which typically amounts to about $9 \%$ of the sum [47].

Important information about the polarized parton distributions can be extracted from the measured structure functions via (5). The most efficient use of the data from the various experiments done at different beam energies and kinematic conditions is achieved by incorporating all available

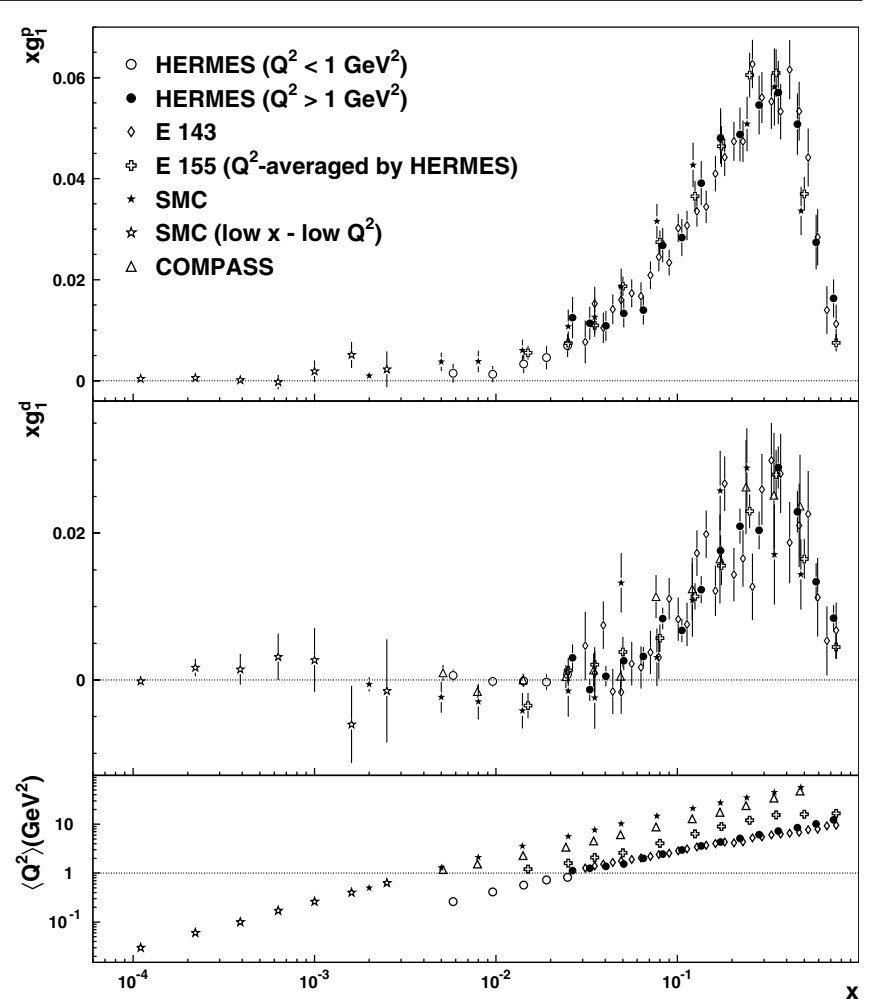

Figure 4. Published experimental results on $x_{\mathrm{B}} \cdot g_{1}^{\mathrm{p}}$ and $x_{\mathrm{B}} \cdot g_{1}^{d}$ versus $x_{\mathrm{B}}$, shown on separate panels, from HeRMEs [47] at DESY, SMC [40-42] at CERN, E143 [31] and E155 [33,34] at SLAC and COMPASS [48] at CERN. Error bars represent the sum in quadrature of statistical and systematic uncertainties. The HERMES data points shown are statistically correlated by unfolding QED radiative and detector smearing effects; the statistical uncertainties shown are obtained from the diagonal elements of their covariance matrix, only. The E143 and E155 data points are correlated through QED radiative corrections. The lower panel shows the $x_{\mathrm{B}}$ dependence of the mean values $\left\langle Q^{2}\right\rangle$ for the various experiments. Reprinted with permission from [47]. Copyright (2006) by the American Physical Society.

data in a global fit based on the evolution predicted by QCD in NLO in $\alpha_{s}$. This approach has the important advantage that it also provides information about the polarization of the gluons contained in the nucleon. In the order $\alpha_{s}$ version of the DGLAP evolution equations, the gluons couple with the quarks via 'splitting': $g \rightarrow q \bar{q}$. Hence the evolution of the $g_{1}$ structure functions with $Q^{2}$ contains information on the helicity density of the gluons. However, in NLO some choice must be made between various 'factorization schemes' for sharing a dependence on $Q^{2}$ between the parton distributions and the coefficient functions relating them to the structure functions. This introduces an arbitrary scheme dependence to the results of the fit, although results obtained in any one scheme can be transformed into any other scheme. The results presented here were obtained in the so-called $\overline{\mathrm{MS}}$ scheme [52].

The fitted parameters appear in some functional form for the $x$ dependence of each of several parton helicity densities, including the gluon helicity density $\Delta G(x)$, at some arbitrarily chosen 'starting' value $Q_{0}^{2}$. (While this functional form is chosen with the benefit of some physical insight, the choice introduces some model dependence to the results from the fit.) At each step in the parameter search, the $g_{1}\left(x_{\mathrm{B}}\right)$ function 


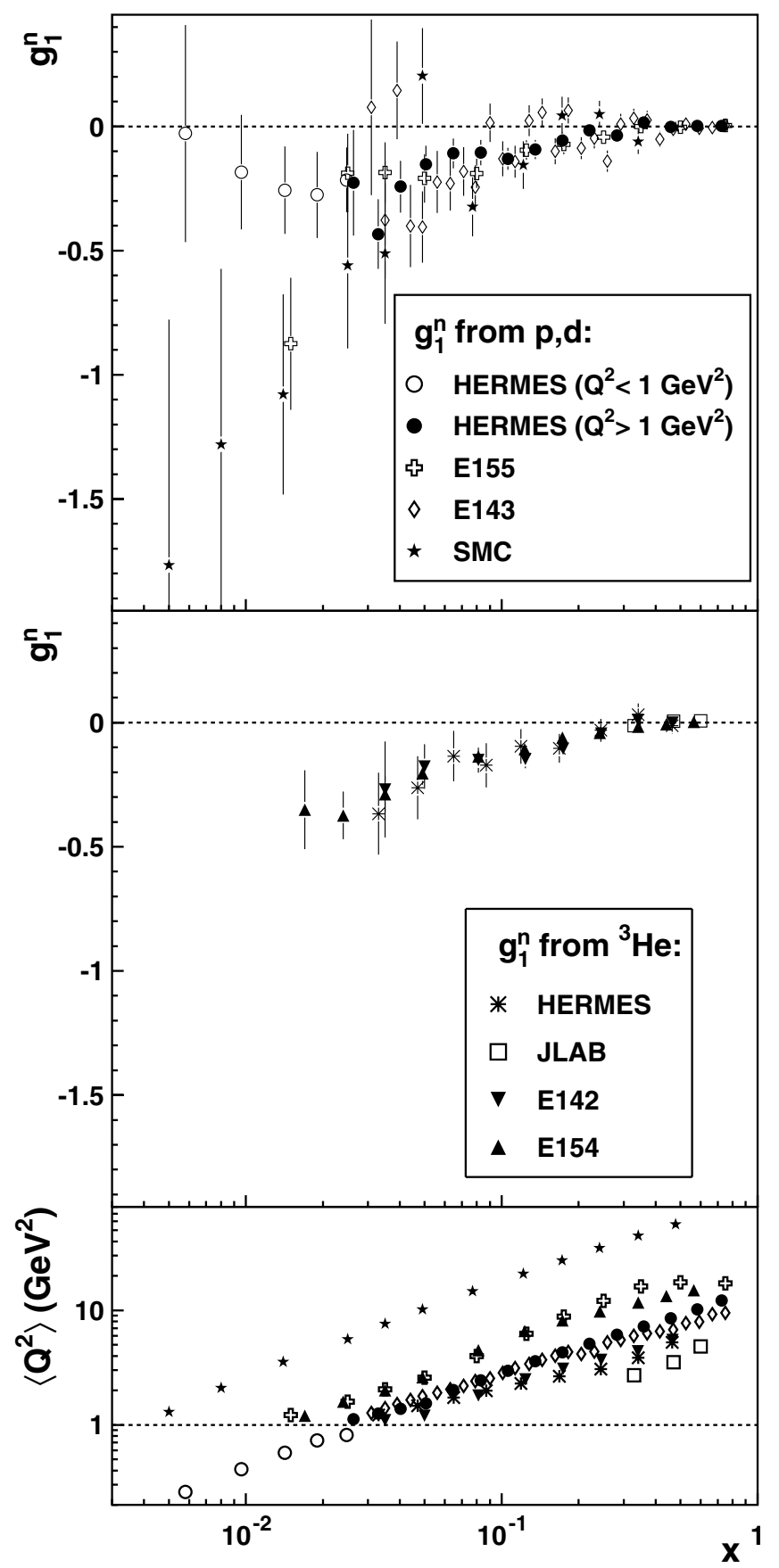

Figure 5. Top panel: the structure function $x_{\mathrm{B}} \cdot g_{1}^{\mathrm{n}}$ obtained from $g_{1}^{\mathrm{p}}$ and $g_{1}^{\mathrm{d}}$, from Hermes [47], SMC [40-42], E143 [31] and E155 $[33,34]$. Second panel from the top: $x_{\mathrm{B}} \cdot g_{1}^{\mathrm{n}}$ obtained from a ${ }^{3} \mathrm{He}$ target [45, 49-51]. Total error bars are shown, obtained by combining statistical and systematic uncertainties in quadrature. The bottom panel shows the $\left\langle Q^{2}\right\rangle$ of each data point in the top two panels. E155 data have been averaged over their $Q^{2}$ bins for visibility. Reprinted with permission from [47]. Copyright (2006) by the American Physical Society.

defined by the current values of the parameters is evolved using the DGLAP equations to the $Q^{2}$ value of each experimental data point, and the discrepancy is taken as a $\chi^{2}$ contribution.

Since the inclusive cross section is sensitive to only the square of the quark charges and not to their signs, inclusive measurements cannot distinguish quarks from antiquarks.
Because of this as well as the limited precision and $Q^{2}$ range of the existing data, a useful fit is impossible without further assumptions. The global fits done up to now include the previously mentioned constraint based on hyperon beta decay lifetimes together with the assumption of SU(3) flavour symmetry, and sometimes also impose the BSR. The strong coupling constant $\alpha_{s}$ may be taken from other experimental information, or may be also fitted as a parameter, in which case it is found to be consistent with previous knowledge. Some fits also impose an (arbitrary) symmetry condition among the sea quarks: $\Delta \bar{u}=\Delta \bar{d}=\Delta s=\Delta \bar{s}$. This is only a device for defining valence quark densities $\Delta q_{f}^{v} \equiv \Delta q_{f}-\Delta \bar{q}_{f}$, and does not affect the fitted strange sea quark and gluon densities.

Many groups have presented results of NLO global fits of all available $g_{1}$ data available at that time. Relatively recent examples are reported in [34,53-57]. Figure 6 shows the results of one recent fit [53] that accounts for contributions to $g_{1}$ that scale as $1 / Q^{2}$, which are significant for the data measured at $Q^{2}$ less than a few $\mathrm{GeV}^{2}$. As the photoabsorption cross section is independent of the sign of the quark charge, $\Delta \bar{u}$ and $\Delta \bar{d}$ are unconstrained by the inclusive data. Hence for this fit the convention was adopted of a flavour symmetric sea:

$$
\Delta u_{\mathrm{sea}}=\Delta \bar{u}=\Delta d_{\mathrm{sea}}=\Delta \bar{d}=\Delta s=\Delta \bar{s} .
$$

It is clearly determined that up quarks have a substantial positive polarization over the entire measured $x$ range, while down quarks have a somewhat smaller and negative polarization. The polarization of the strange quark sea is found to be small but clearly negative. The gluon helicity distribution $\Delta G(x)$ is still poorly constrained, even though many new precise data have recently been added to the global set. In fact there are two alternative solutions (local minima in $\chi^{2}$ ) of equal quality with opposite signs of the gluon density, and yet another of similar quality using a bipolar shape for the $x$ dependence. The reason for the large uncertainty is that the $g_{1}$ data set is still far less extensive and precise than the unpolarized set, and covers a much more limited range in $Q^{2}$ (see section 2.8). The first moments of the helicity densities determined from the fit are shown in table 1. (These moments have some sensitivity to the assumed functional form of the densities, especially in the unmeasured small- $x$ region.) The flavour-singlet moment $\Delta \Sigma$ is found to be in the range $0.2-0.25$, implying that the total $\frac{1}{2}(1-\Delta \Sigma)$ of the remaining contributions to the spin of the nucleon from the gluons and/or quark orbital angular momenta is substantial. The gluon moment $\Delta G$ is still poorly determined, but it appears that large values are disfavoured.

This collection of increasingly precise data from all of the experiments also offers the opportunity to more severely test the BSR. It is difficult to efficiently employ all of the data while computing the sum directly from the measured values of $g_{1}^{\mathrm{p}}$ and $g_{1}^{\mathrm{n}}$ (or $g_{1}^{\mathrm{d}}$ ) using (8), because kinematic interpolation and evolution to a common value of $Q^{2}$ is needed. This in turn requires a parametrization of the $x_{\mathrm{B}}$ dependence at some 'starting' value of $Q^{2}$, just as in the fits described above. In fact, those fits can be used to test the BSR, simply by omitting the usual BSR constraint from the fit, and computing the sum from the resulting $\Delta q(x)$ distributions produced by the fit (while accounting for uncertainty correlations). The relevant 

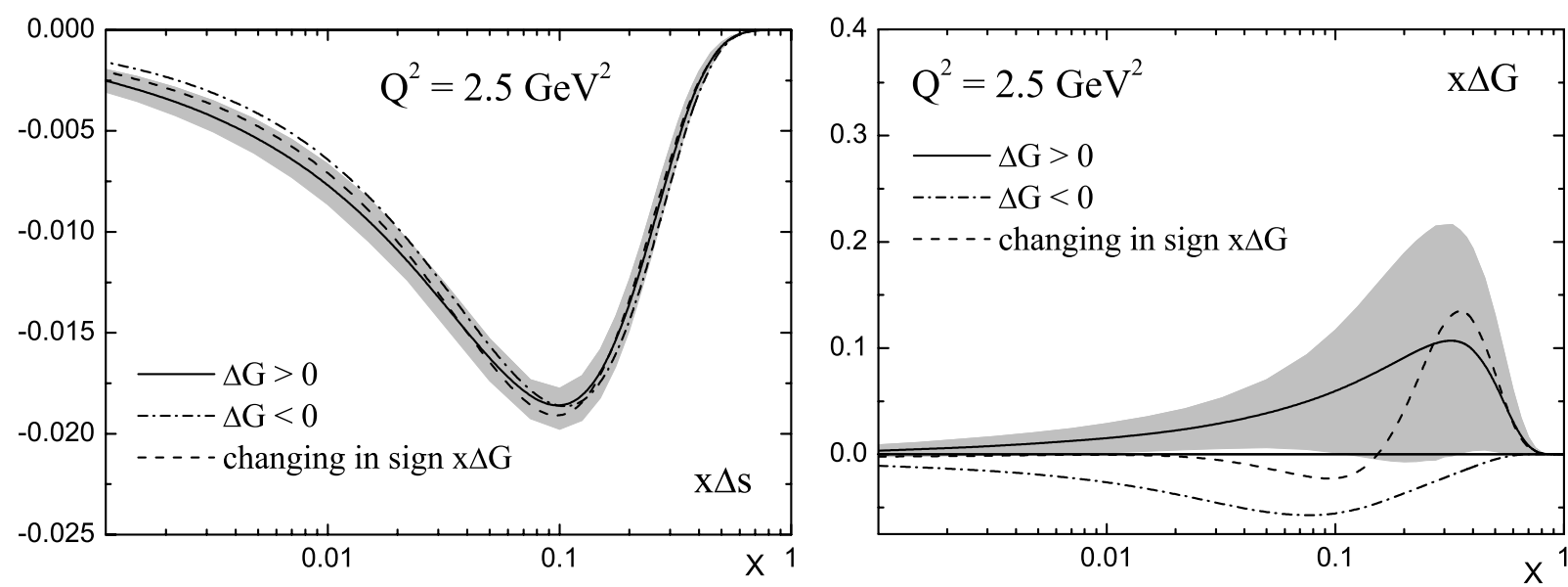

Figure 6. Helicity densities of the strange sea $\Delta s(x)+\Delta \bar{s}(x)$ (left) and of the gluons (right), at $Q^{2}=2.5 \mathrm{GeV}^{2}$, for three different choices for the shape of the gluon densities, all with a similar quality of fit to the $g_{1}$ data. Reprinted with permission from [53]. Copyright (2007) by the American Physical Society.

Table 1. First moments of the helicity densities resulting from the NLO QCD fit to all available $g_{1}$ data of [53] in the ( $\left.\overline{\mathrm{MS}}\right)$ factorization scheme, at the input scale of $Q^{2}=1 \mathrm{GeV}^{2}$. The uncertainties shown are total (statistical and systematic combined in quadrature). The parameters marked by $(*)$ are fixed by the assumption of SU(3) flavour symmetry mentioned in the text. The BSR is also enforced. The table is taken from [53].

\begin{tabular}{lcc}
\hline & $\Delta G>0$ & $\Delta G<0$ \\
\hline DoF & $826-20$ & $826-20$ \\
$\chi^{2}$ & 721.7 & 722.9 \\
$\chi^{2} / \mathrm{DoF}$ & 0.895 & 0.897 \\
$\Delta u_{v} \equiv \Delta u-\Delta \bar{u}$ & $0.926^{*}$ & $0.926^{*}$ \\
$\Delta d_{v} \equiv \Delta d-\Delta \bar{d}$ & $-0.341^{*}$ & $-0.341^{*}$ \\
$\Delta \Sigma$ & $0.207 \pm 0.040$ & $0.243 \pm 0.065$ \\
$\Delta s+\Delta \bar{s}$ & $-0.063 \pm 0.005$ & $-0.057 \pm 0.010$ \\
$\Delta G$ & $0.129 \pm 0.166$ & $-0.200 \pm 0.414$ \\
\hline
\end{tabular}

combination providing the structure function difference is the 'flavour non-singlet' distribution:

$$
\Delta q_{\mathrm{NS}}(x) \equiv \sum_{f}\left(e_{f}^{2} /\left\langle e^{2}\right\rangle-1\right)\left(\Delta q_{f}(x)+\Delta \bar{q}_{f}(x)\right) .
$$

The removal of the BSR constraint from the fit may result in large uncertainties for individual densities, but their uncertainty correlations should result in a smaller uncertainty for $\Delta q_{\mathrm{NS}}$. This approach might at first seem bizarre, because the sum rule is believed to be much more reliable than the assumption of SU(3) flavour symmetry used in the fits! However, it can be expected that $\Delta q_{\mathrm{NS}}$ will be insensitive to the constraint from hyperon decay data if the proton and deuteron data have comparable precision and kinematic coverage, which is the case. (This can easily be verified by varying this input to the fit to study the sensitivity.) Hence for this purpose, the fitting process provides mainly the evolution of the data points to a common value of $Q^{2}$, as well as the model used to extrapolate to $x=0,1$. This approach leads to good agreement with the BSR within the total uncertainty from the fit, which amounted to about $10 \%$ of the sum, using the data available in 1998 [58]. Much more precise data are available today.
There are several technical issues arising in the QCD fits that can be kept in mind when considering their results. Firstly, typically the values taken from experiment are those for $g_{1} / F_{1}$, even though the unpolarized structure function $F_{1}$ has no relevance, and the systematic uncertainties for $g_{1} / F_{1}$ include a significant contribution from the poorly known ratio $R=\sigma_{\mathrm{L}} / \sigma_{\mathrm{T}}$ of cross sections for longitudinal and transverse photons, which has no effect on $g_{1}$ itself. The reason given for this choice is the fact that experimental groups may have extracted $g_{1}$ from the measured asymmetries effectively using values for the unpolarized cross section that were calculated using various values of $R$. However, as it is the ratio $g_{1} / F_{1}$ that is sensitive to $R$ and not $g_{1}$, it appears that this choice aggravates the problem. (Fortunately, the effects of this may be small.) Secondly, it can be difficult to obtain reliable results from multidimensional fits of data with limited statistical accuracy. Evidence for such difficulty here is the irregular shape of the dependence of $\chi^{2}$ on some parameters that are reported. Up to three minima have recently been seen with similar values of $\chi^{2}$ [53]. A common method of testing the stability and accuracy of algorithms for extracting results from experimental data is to analyse Monte Carlo pseudo-data. The situation here could be clarified if $g_{1}$ pseudo-data were generated from a chosen set of polarized parton distributions, which could be compared with the results of the fit. Thirdly, two groups report statistical uncertainties that are inflated by approximately the square root of the number of fitted parameters (assuming that the $\chi^{2}$ surface is quadratic in a substantial region near the minimum), so that their uncertainties cannot be directly compared with those of other groups. These inflated uncertainties do not correspond to what is understood by most of the community as a statistical uncertainty. (One way of defining this conventional uncertainty is the standard deviation of the distribution of the results that would be derived by similarly fitting a large number of Monte Carlo data sets resembling the global experimental data set, but with each data point fluctuating independently according to its experimental statistical uncertainty.) Fourthly, some groups account for the (substantial) experimental normalization uncertainty that 
is common to an entire data set from one experiment (arising from, e.g., uncertainty in polarization of beam or target) by adding that common component of the systematic uncertainty in quadrature to the statistical uncertainty of each data point. This incorrectly deprecates the information available in those data. It is straightforward to more appropriately account for the normalization uncertainty by means of a $\chi^{2}$ penalty term driven by a renormalization factor for that data set. Fifthly, few groups (see e.g. [55,58]) estimate 'theoretical' uncertainties for their results, arising from, e.g., the somewhat arbitrary choice of functional forms to parametrize the helicity densities, and the dependence of the results on factorization and renormalization scales, indicating incomplete convergence of the perturbative expansion.

We have seen that the global data set for the spin structure function $g_{1}$ on the proton and neutron target has firmly established that quark helicities account for only a fraction of the proton spin, leaving substantial contributions to be made by gluons and quark orbital angular momenta. The large uncertainty in the moment $\Delta G$ from global fits to $g_{1}$ data has motivated several major efforts to directly measure $\Delta G(x)$ using processes in which gluons enter in leading order, as described in section 2.8. Furthermore, information about quark orbital angular momentum is beginning to emerge from both calculations on the lattice and measurements of cross section differences or asymmetries for the DVCS process, as described in sections 4 and 5.2.

\subsection{Helicity densities of sea quarks from semi-inclusive DIS data}

As mentioned above, quarks and antiquarks of the same flavour cannot be distinguished in inclusive DIS measurements, since the contributions of the various quark flavours to the cross sections scale as the square of the quark charges and hence are independent of the sign. Furthermore, the strange sea can be separated only in terms of first $x$-moments, because of the necessary constraint from hyperon beta decay data. In order to obtain information about the $x$ dependence of the helicity densities of the various flavours, particularly of sea quarks, it is necessary to use additional types of experimental information. In semi-inclusive scattering (see figure 1), an energetic hadron is detected in coincidence with the scattered lepton. If suitable kinematic criteria are applied in the selection of the hadrons, they are likely to be members of the 'jet' of hadrons produced in the fragmentation of the struck quark, the non-perturbative process in which the energy of the quark is shared among hadrons created through the excitation of quark-antiquark pairs from the vacuum. For our purposes here, a key point is that the identities of the hadrons are statistically correlated with the flavour of the struck quark. For example, a $\pi^{+}$meson having valence composition $u \bar{d}$ is more likely to appear in the fragmentation of a $u$ or $\bar{d}$ struck quark, and kaons are more likely to arise from strange quark fragmentation. However, these probabilities are also modulated by the square of the charges of the various flavours and their (known) spin-averaged quark densities. Hence the exploitation of this method is limited to some degree by the ' $u$-quark dominance' arising from both of these effects. For example, $\pi^{-}=d \bar{u}$ mesons and even $\mathrm{K}^{-}=\bar{u} s$ mesons, which provide good sensitivity to sea quarks, arise more often from fragmentation of $u$ quarks. Hence a quark flavour decomposition based on data for various identified hadrons requires the solution of a strongly coupled system of relationships, which in turn requires primary data of high precision.

Given an adequate understanding of the fragmentation process, a complete flavour decomposition of the quark and antiquark helicity distributions can be extracted from sufficiently precise measurements on both proton and 'neutron' targets of double-spin asymmetries in the cross sections for leptoproduction of various types of hadrons. The extraction requires knowledge of the probabilities for the various types $h$ of hadrons to appear in the fragmentation of a struck quark of a given flavour $f$. These probabilities are embodied in the (familiar unpolarized) fragmentation functions $D_{f}^{\mathrm{h}}(z)$, where $z \equiv E_{\mathrm{h}} / v$ and $v$ and $E_{\mathrm{h}}$ are the energies in the target rest frame of the absorbed virtual photon (and hence of the struck quark) and of the detected hadron. While these fragmentation functions have typically been extracted $[59,60]$ from mostly high-energy $\mathrm{e}^{+} \mathrm{e}^{-}$collider data, a recent extraction [61] includes preliminary semi-inclusive DIS multiplicities of identified hadrons [62].

For leptoproduction of a hadron $\mathrm{h}$, the semi-inclusive cross section difference that is analogous to the inclusive case of (2) can be factorized in leading order in $\alpha_{s}$ as

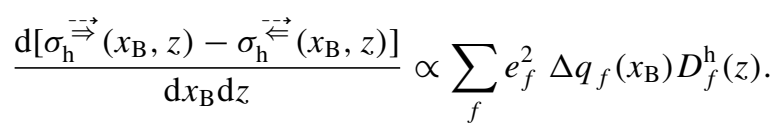

If, for example, there are available experimental measurements of these cross section differences for production of $\pi^{+}, \pi^{-}$, $\mathrm{K}^{+}$and $\mathrm{K}^{-}$mesons on both proton and 'neutron' targets, this system of eight over-constrained equations can, in principle, be solved for the helicity densities of up to six light quark flavours. As mentioned above, in practice $u$-quark dominance limits the sensitivity to some sea quark flavours.

As in the case of inclusive measurements, absolute cross section differences are difficult to measure, especially with polarized targets. Hence cross section asymmetries are extracted from the data. Figure 7 shows all of the measured semi-inclusive double-spin asymmetries on both hydrogen and deuterium targets. It includes data from SMC for unidentified hadrons [43], from HERMEs for production of identified $\pi^{+}, \pi^{-}$, $\mathrm{K}^{+}$and $\mathrm{K}^{-}$mesons [63], and from Compass for unidentified hadrons [64]. Each collaboration extracted helicity densities in leading order in $\alpha_{s}$. In the case of HERMEs, all three sea quark flavours could be distinguished, while fragmentation functions were effectively fitted to hadron multiplicities measured by the same experiment. It was found that the helicity densities of all three sea quark flavours are consistent with zero within the measured $x$ range. Further analysis of all these data was done later in NLO, including data from other processes. This is described in the next section. 


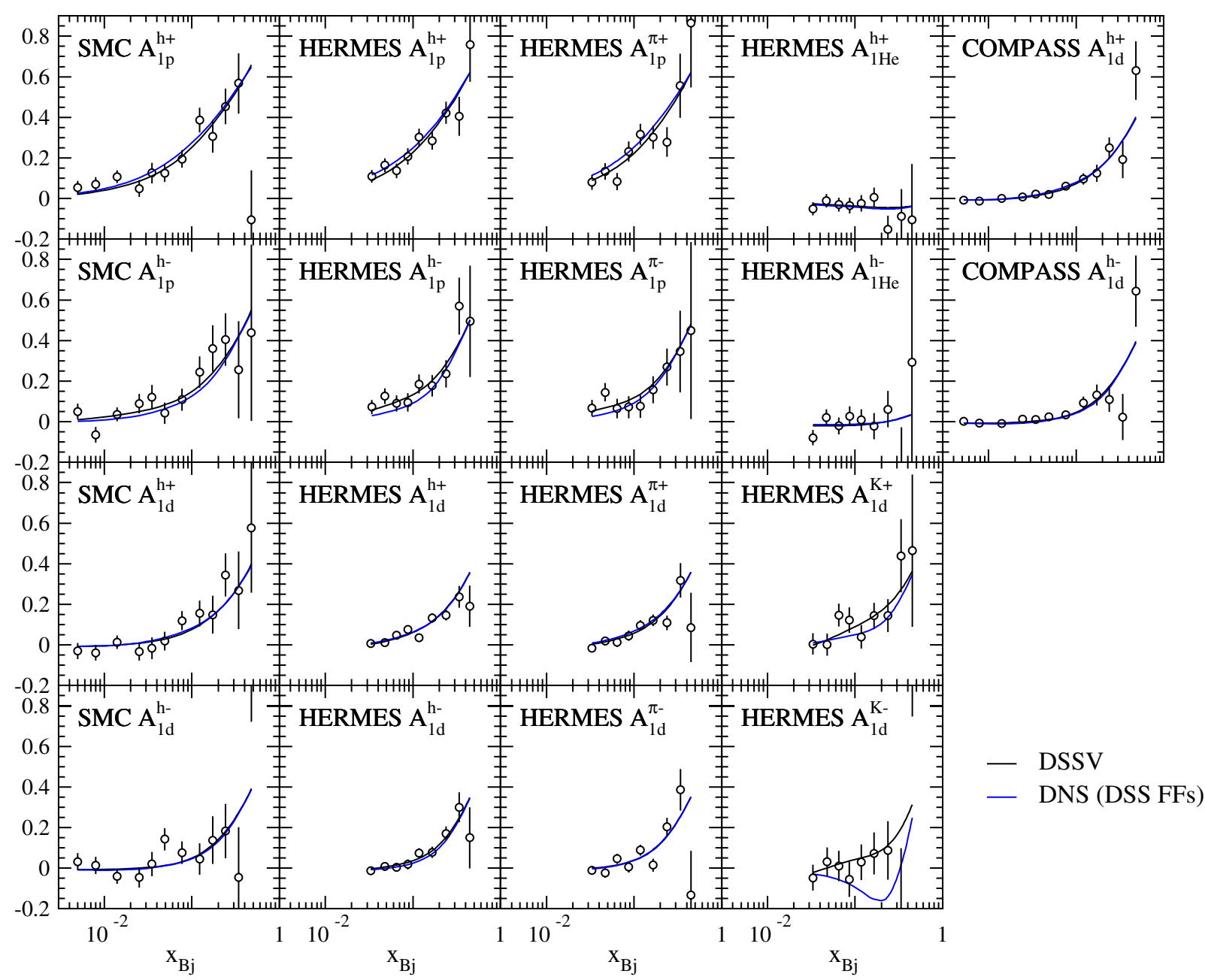

Figure 7. Semi-inclusive asymmetries for virtual-photon-nucleon DIS on both the proton (p) and deuteron (d), producing the type of hadron labelled by the superscript on the symbols in the figure (' $h$ ' means unidentified hadrons). These data from SMC [43], HERMES [63] and Compass [64] are compared with curves computed from helicity densities fitted to these and other data in NLO [65]. The acronym 'DNS' refers to the previous fit by this group [56,66] using older information [67] about fragmentation functions. Reprinted with permission from [65]. Copyright (2008) by the American Physical Society.

\subsection{Helicity densities from a global analysis in NLO}

The most complete extraction of helicity densities from all relevant published data appears in [65]. It is a global NLO QCD analysis like that described in section 2.4 for inclusive DIS data, but with the crucial inclusion of semi-inclusive DIS data to provide sensitivity to individual sea quark flavours, and for the first time RHIC $\mathrm{p}^{\Rightarrow}+\mathrm{p} \Rightarrow$ data for inclusive production of neutral pions and jets, providing much stronger constraints on the gluon helicity. Another new feature of the fit is the use of the above-mentioned set of fragmentation functions [61] based on not only $\mathrm{e}^{+} \mathrm{e}^{-}$data but also preliminary semi-inclusive DIS multiplicities of identified hadrons.

The extensive data set allows some freedom in the application of the constraint of neutron [hyperon] beta decay data through the assumption of $\mathrm{SU}(2)$ [SU(3)] flavour symmetry. Penalty terms were added to $\chi^{2}$ to limit violations of SU(2) and SU(3) symmetries, but their values at the solution are negligible compared with unity, suggesting that, at least in terms of first moments, the data are fully compatible with these symmetries. Because the data are still unable to distinguish $s$ and $\bar{s}$ flavours, the assumption is made that $\Delta s=\Delta \bar{s}$. (A previous analysis [63] in LO found that only $\Delta s$ is constrained by the HERMES data, and the above assumption has negligible effect on that analysis.)

Well-defined unique minima appear in the dependence of $\chi^{2}$ on each of the three sea quark flavours, and the quality of the fit is excellent: $\chi^{2} / \mathrm{DoF}=0.88$. Figure 7 shows a comparison of the resulting fits with the available semi-inclusive asymmetries. Asymmetries calculated from the same fragmentation functions but with helicity densities from a previous fit based on an older parametrization [67] of fragmentation functions are also shown, illustrating the sensitivity of the kaon asymmetries and hence sea densities on this input to the fit.

Figure 8 shows the resulting parton helicity densities with uncertainty bands, while table 2 shows their (truncated) first moments. The densities $\Delta u+\Delta \bar{u}$ and $\Delta d+\Delta \bar{d}$ are not shown because they were already well constrained by the inclusive data. The semi-inclusive data constrain the sea densities, especially $\Delta s$, where a solution to a long-standing mystery appears. Leading-order analysis of semi-inclusive data has 


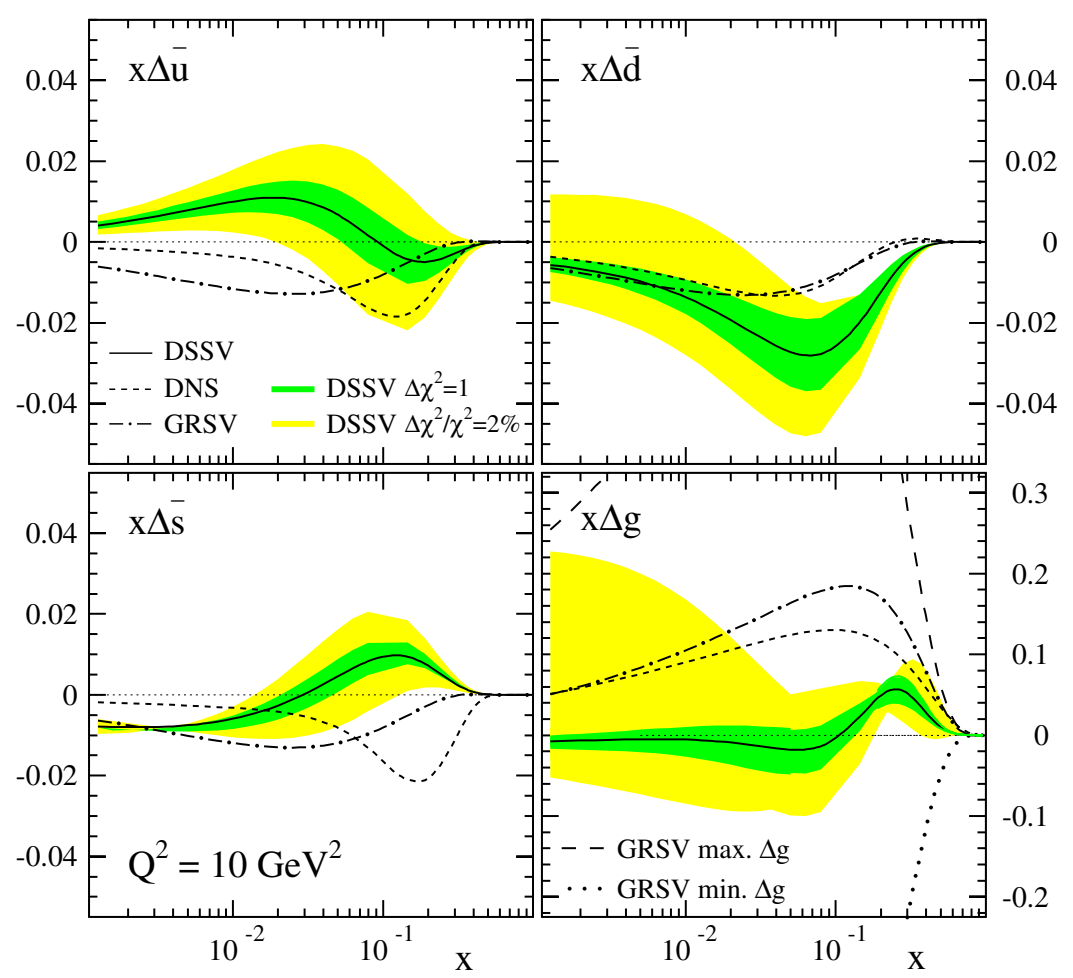

Figure 8. The continuous and dashed curves represent parton helicity densities multiplied by $x$, extracted in NLO from double-spin DIS and $\mathrm{p}^{\Rightarrow}+\mathrm{p} \Rightarrow$ data and evaluated at $Q^{2}=10 \mathrm{GeV}^{2}$, with their inner and outer uncertainty bands corresponding to $\delta \chi^{2}=1$ and $\delta \chi^{2}=2 \%$ of $\chi^{2}$, respectively. The dotted and long-dashed curves represent the previously known unpolarized densities, which act as positivity limits to the helicity density limits through the requirement that all cross sections be non-negative. The dashed (dot-dashed) curves represent the results of the previous fits of [56] ([54]). Reprinted with permission from [65]. Copyright (2008) by the American Physical Society.

Table 2. Truncated first moments $\int_{x_{\min }}^{1} \mathrm{~d} x \Delta q\left(x, Q^{2}\right)$ at $Q^{2}=10 \mathrm{GeV}^{2}$, taken from [65]. It is important to note the factor of two relating $\Delta \bar{s}$ in this table (assuming $\Delta s=\Delta \bar{s}$ ) to $\Delta s+\Delta \bar{s}$ in table 1 . The uncertainties for $\Delta \chi^{2}=1$ account only for experimental statistical and systematic uncertainties added in quadrature, and not for 'theoretical' or model uncertainties.

\begin{tabular}{lrrr}
\hline & $x_{\min }=0$ & \multicolumn{2}{c}{$x_{\min }=0.001$} \\
\cline { 3 - 4 } & Best fit & \multicolumn{1}{c}{$\Delta \chi^{2}=1$} & \multicolumn{1}{c}{$\Delta \chi^{2} / \chi^{2}=2 \%$} \\
\hline$\Delta u+\Delta \bar{u}$ & 0.813 & $0.793_{-0.012}^{+0.011}$ & $0.793_{-0.034}^{+0.028}$ \\
$\Delta d+\Delta \bar{d}$ & -0.458 & $-0.416_{-0.009}^{+0.011}$ & $-0.416_{-0.025}^{+0.035}$ \\
$\Delta \bar{u}$ & 0.036 & $0.028_{-0.020}^{+0.021}$ & $0.028_{-0.059}^{+0.059}$ \\
$\Delta \bar{d}$ & -0.115 & $-0.089_{-0.029}^{+0.029}$ & $-0.089_{-0.080}^{+0.090}$ \\
$\Delta \bar{s}$ & -0.057 & $-0.006_{-0.012}^{+0.010}$ & $-0.006_{-0.031}^{+0.028}$ \\
$\Delta G$ & -0.084 & $0.013_{-0.120}^{+0.106}$ & $0.013_{-0.314}^{+0.702}$ \\
$\Delta \Sigma$ & 0.242 & $0.366_{-0.018}^{+0.015}$ & $0.366_{-0.062}^{+0.042}$ \\
\hline
\end{tabular}

indicated that the strange helicity density is small or even positive at large $x$, whereas all analyses of only inclusive data imposing SU(3) symmetry clearly favour negative values of the first moment of $\Delta s+\Delta \bar{s}$. These findings are now reconciled by a bipolar shape of $\Delta \bar{s}$ that is negative at small $x$ and positive at large $x$. However, figure 8 shows that the first moment $\Delta \bar{s}$ is negative only because of the substantial contribution from the region $x<0.01$, and table 2 shows that even $x<0.001$ contributes strongly. It may seem mysterious that the fit forces $\Delta \bar{s}$ to be negative in a region of small $x$ devoid of data, while the penalty term for $\mathrm{SU}(3)$ violation is so small at the solution point. However, the explanation may be that the $\Delta \bar{s}$ distribution is completely unconstrained in this $x$ range by anything but the penalty term, so a very small value of the penalty is sufficient.

The gluon helicity density is constrained, primarily by the addition of the RHIC data, to be significantly smaller than previous uncertainties, in the experimentally accessible $x$ range. Table 2 shows that the first moment $\Delta G$ of the possibly bipolar distribution is consistent with zero, still with rather large uncertainties.

A quantity of particular interest that is accessible to semiinclusive DIS data is the flavour asymmetry $\Delta \bar{u}(x)-\Delta \bar{d}(x)$. Naively, one might expect that since $u$ and $d$ quarks have similarly small masses and they both are produced by gluon 'splitting' ( $g \rightarrow q \bar{q}$ ), both their densities and polarizations would be similar. However, the difference in their unpolarized densities $\bar{u}(x)-\bar{d}(x)$ is experimentally well established [68], and is explained by various non-perturbative models that also predict an asymmetry in the helicity densities. Such a delicate imbalance in nucleon structure provides an excellent test of such models. The flavour asymmetry $\Delta \bar{u}-\Delta \bar{d}$ in the first moments can be compared with the predictions of two representative models. One is the chiral quark soliton model ( $\chi$ QSM) [69], which is an effective theory where baryons appear as soliton solutions of the chiral Lagrangian, and the other is a 'meson cloud' model [70] that considers quantum fluctuations of the spin- $\frac{1}{2}$ nucleon into a virtual pion with spin 1 plus a $\Delta$ hadron with spin $\frac{3}{2}$. The value $\Delta \bar{u}-\Delta \bar{d}=0.15_{-0.10}^{+0.11}$ from the fit is consistent with the predictions of both models, but with a preference for the $\chi$ QSM. 
Table 3. Recent published values of first moments of helicity densities, separated into experimental evaluations based on the first moment of $g_{1 \mathrm{~d}}$, and evaluations from NLO QCD fits of various overlapping data sets. The DSSV values for $\Delta s+\Delta \bar{s}$ are computed from their fit result for $\Delta \bar{s}$ using their assumption $\Delta s=\Delta \bar{s}$. The fit uncertainties for $\Delta \chi^{2}=1$ account only for experimental, statistical and systematic uncertainties added in quadrature, and not for 'theoretical' or model uncertainties. The DSSV uncertainties apply only to the partial moments over the range $x>0.001$, and hence should be interpreted with care. All evaluations are in the $\overline{\mathrm{MS}}$ scheme.

\begin{tabular}{|c|c|c|c|c|c|}
\hline Analysis & Year & $\begin{array}{l}Q^{2} \\
\left(\mathrm{GeV}^{2}\right)\end{array}$ & $\Delta \Sigma$ & $\Delta s+\Delta \bar{s}$ & $\Delta G$ \\
\hline \multicolumn{6}{|l|}{ Direct experimental evaluations } \\
\hline HeRmes [47] $(d$ target $)$ & 2006 & 5 & $\begin{array}{l}0.330 \pm 0.011 \text { (theor.) } \\
\pm 0.025 \text { (exp.) } \pm 0.028 \text { (evol.) }\end{array}$ & $\begin{array}{l}-0.085 \pm 0.013 \text { (theor.) } \\
\pm 0.008 \text { (exp.) } \pm 0.009 \text { (evol.) }\end{array}$ & - \\
\hline CoMPASS [72] ( $d$ target $)$ & 2006 & 3 & $\begin{array}{l}0.35 \pm 0.03 \text { (stat.) } \\
\pm 0.05 \text { (syst.) }\end{array}$ & $\begin{array}{l}-0.08 \pm 0.01 \text { (stat.) } \\
\pm 0.02 \text { (syst.) }\end{array}$ & - \\
\hline \multicolumn{6}{|l|}{$N L O Q C D$ fits } \\
\hline $\operatorname{AAC}[57]\left(\Delta \chi^{2}=12.65\right)$ & 2006 & 1 & $0.27 \pm 0.07$ & $-0.10 \pm 0.02$ & $0.31 \pm 0.32$ \\
\hline $\operatorname{LSS}[53]\left(\Delta \chi^{2}=1\right)$ & 2006 & 1 & $0.17-0.31$ & $-0.068--0.047$ & $-0.6-0.3$ \\
\hline $\operatorname{DSSV}[65]\left(\Delta \chi^{2}=1\right)$ & 2008 & 1 & $0.242_{-0.018}^{+0.015}$ & $-0.114 \pm 0.02$ & $-0.084_{-0.120}^{+0.106}$ \\
\hline $\operatorname{DSSV}[65]\left(\Delta \chi^{2}=0.02, \chi^{2}=7.85\right)$ & 2008 & 1 & $0.242_{-0.062}^{+0.019}$ & $-0.114 \pm 0.06$ & $-0.084_{-0.314}^{+0.702}$ \\
\hline
\end{tabular}

\subsection{Comparison of first moments of helicity densities}

Two previous sections discussed three significantly different but not independent sources of information about first moments of helicity distributions: the direct interpretation of the first moment of the spin structure function $g_{1}^{\mathrm{d}}$ of the deuteron, NLO QCD fits of parametrized helicity densities to all $g_{1}$ data (see section 2.4) and global fits of helicity densities to all available relevant data, including double-spin asymmetries for both semi-inclusive DIS and inclusive production of neutral pions and jets in $\mathrm{p}^{\Rightarrow}+\mathrm{p} \Rightarrow$ (section 2.6). The first moments from these analyses are compared in table 3 . The comparison of results from the NLO QCD fits should be undertaken with caution, as the analyses differ in several respects. The LSS fit uses only inclusive DIS data, including the Compass data on the deuteron, which leads to two equally probable solutions. They are merged into a range of values given in the table rather than central values with standard deviations. The AAC fit does not yet include the Compass data, but includes some double-spin asymmetries for inclusive production of neutral pions in $\mathrm{p} \Rightarrow+\mathrm{p} \Rightarrow$. Finally the DSSV fit includes all inclusive and semi-inclusive DIS data, as well as doublespin asymmetries for both semi-inclusive DIS and inclusive production of neutral pions and jets in $p^{\Rightarrow}+p^{\Rightarrow}$. Another important difference between the analyses is the treatment of experimental statistical uncertainties, indicated in the first column. The LSS uncertainties are based on the standard treatment specified by the PDG [71], while the AAC group adopts a different treatment that increases their uncertainties. The DSSV group provides the results from two treatments. Finally, the common elements of the data sets used for the various results imply that the uncertainties are correlated, so that they should, in principle, be in better agreement than indicated by their uncertainties.

The values for $\Delta \Sigma$ are all consistent, with the possible exception that the DSSV result for the 'standard' treatment of statistical uncertainties $\left(\Delta \chi^{2}=1\right)$ appears to be somewhat smaller. However it is important to bear in mind that the contribution -0.124 to that result from the unmeasured region $x<0.001$ is much larger than this 'discrepancy'.
Having accounted for the factor of two relating the DSSV value for $\Delta \bar{s}$ (assuming $\Delta s=\Delta \bar{s}$ ) to the values for $\Delta s+\Delta \bar{s}$ from the other two fits, all of the results again are consistent, again with the possible exception that the DSSV result for $\Delta \chi^{2}=1$ appears to be somewhat more negative. However, essentially all of this value is contributed by the unmeasured region $x<0.001$. The uncertainty of the DSSV result is much larger than that of AAC, in spite of the additional data used in the DSSV fit, presumably because of the relaxation of SU(3) flavour symmetry.

Finally, all the results for $\Delta G$ are consistent with each other and with zero. The additional RHIC data included in the DSSV fit resolve the ambiguity arising in the LSS fit, and much improve the precision. Here the contribution to that result from the unmeasured region is about -0.07 . Other (leading order) experimental constraints on $\Delta G$ are discussed in the next subsection.

\subsection{Gluon helicity distribution}

The nucleon spin can be decomposed into contributions of quarks and gluons, as will be explained in section 5.1. A decomposition in which all terms have a probabilistic interpretation as parton densities in the helicity basis can be written as

$$
\frac{1}{2}=\frac{1}{2} \Delta \Sigma+\Delta G+\mathcal{L}
$$

where $\mathcal{L}$ is the total orbital angular momentum of partons. The intrinsic gluon contribution $\Delta G$ is accessible to experiment. Until recently the only existing knowledge about the gluon helicity distribution $\Delta G(x)$ was derived as its first moment $\Delta G \equiv \int_{0}^{1} \mathrm{~d} x \Delta G(x)$, from NLO pQCD fits to the spindependent structure function $g_{1}\left(x, Q^{2}\right)$ measured in doublespin lepton-nucleon scattering experiments (see section 2.3). However, this method is subject to large uncertainties, as $g_{1}\left(x, Q^{2}\right)$ is only weakly sensitive to $\Delta G(x)$ via the $Q^{2}$ evolution of PDFs. Very recently, better precision was achieved in a global analysis of DIS and pp data, as explained in section 2.6. 


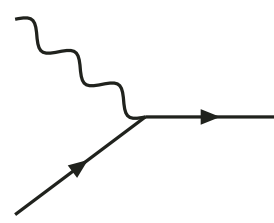

(a)

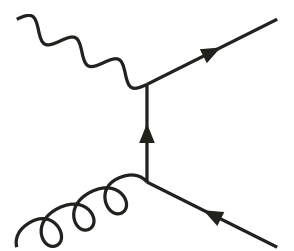

(b)

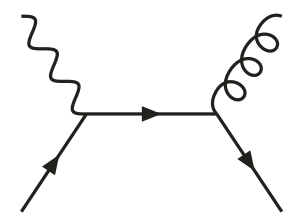

(c)

Figure 9. Feynman diagrams for hard subprocesses in lepton-nucleon scattering: $(a) \mathcal{O}\left(\alpha_{s}^{0}\right)$ DIS $\left(\gamma^{*} q \rightarrow q\right),(b) \mathcal{O}\left(\alpha_{s}^{1}\right)$ PGF $\left(\gamma^{*} g \rightarrow q \bar{q}\right)$, (c) $\mathcal{O}\left(\alpha_{s}^{1}\right)$ QCD Compton scattering $\left(\gamma^{*} q \rightarrow q g\right)$.

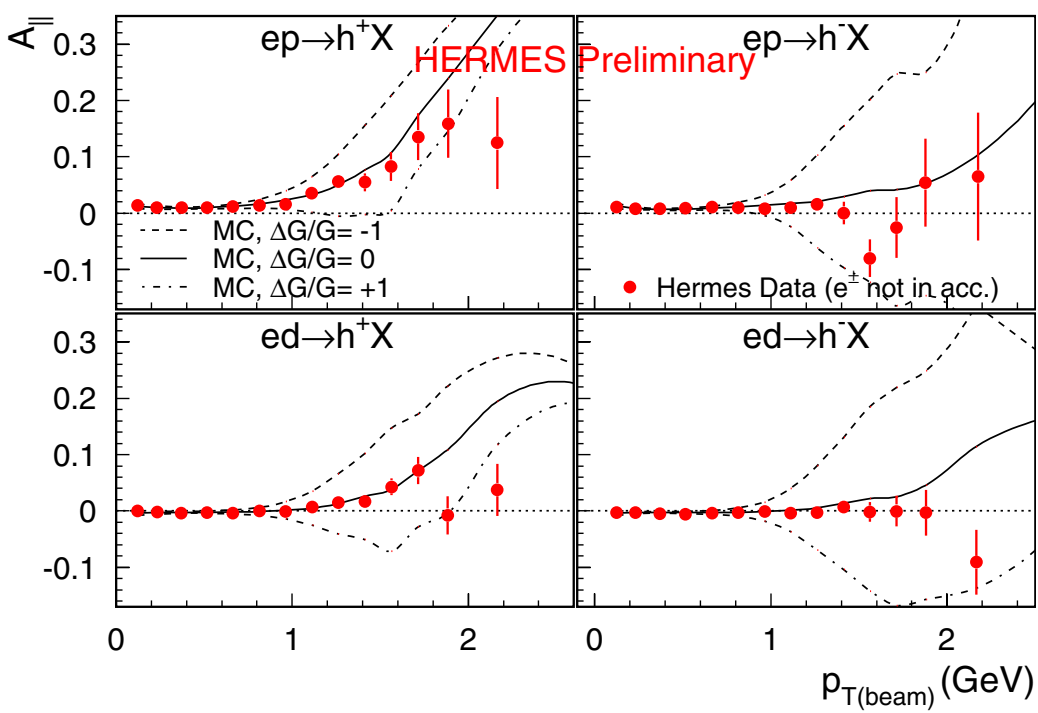

Figure 10. Asymmetry measured at Hermes for positive (left) and negative (right) inclusive charged hadrons on the proton (top) and deuteron (bottom), as a function of $p_{\mathrm{T}(\text { beam) }}$. The errors are statistical only. There is an overall experimental normalization of 5.2\% (3.9\%) for proton (deuteron) data. The curves show the asymmetries as expected from the PYтнIA [73, 74] Monte Carlo simulation for three different assumptions on the gluon polarization. Reprinted with permission from [75]. Copyright (2007), American Institute of Physics.

More experimental data are now emerging from the study of the subprocess in which gluons appear in leading order in double-spin lepton-nucleon scattering: photon-gluon fusion (PGF), see figure $9(b)$. Here, the gluon emits a $q \bar{q}$ pair in which each quark inherits the helicity of the gluon. As explained in section 1, the (anti-)quark can absorb the photon only if they have the same helicity. Given positive (negative) values of $\Delta G(x)$, the PGF process has a negative (positive) asymmetry, while the major background processes DIS (figure 9(a)) and QCD Compton (figure $9(c)$ ) have always a positive asymmetry.

The PGF process produces a $q \bar{q}$ pair resulting in two jets of hadrons. At fixed-target energies, one has to use a leading hadron to represent a jet, plus possibly another hadron separated by a large transverse momentum $k_{\mathrm{T}}$ to represent the other jet. In order to suppress background processes, final states are selected which contain either large transverse momenta or charm. These signatures are also exploited to access $\Delta G(x)$ in double-spin proton-proton scattering in the experiments STAR and PHENIX at RHIC. A cleaner channel in these experiments will be single direct photon production.

The gluon polarization $(\Delta G / G)(x)$ is extracted in a leading-order analysis from the measured double-spin asymmetry $A_{\|}$in the hadron production cross section. Results on $(\Delta G / G)(x)$ are available from SMC, Compass and Hermes. In particular, COMPASS uses charmed meson decay products and pairs of high- $k_{\mathrm{T}}$ hadrons, while Hermes uses single charged high- $k_{\mathrm{T}}$ hadrons. The hard scale is given by the charm quark mass in the former, and by $k_{\mathrm{T}}$ in the latter case. Using as an example HeRmes data for $h^{ \pm}$-production on the proton and deuteron, respectively [75], $A_{\|}$is shown in figure 10 as a function of hadron transverse momentum $p_{\mathrm{T} \text { (beam) }}$ that is measured with respect to the beam axis. Also shown is the dependence expected from a Monte Carlo simulation for several values of $(\Delta G / G)(0, \pm 1)$, which demonstrates that for $p_{\mathrm{T}(\text { beam })}>1 \mathrm{GeV}$ the asymmetry clearly starts to depend on the underlying value for $\Delta G / G$. Comparing it with the data reveals, at the level of the asymmetry, that small values of $\Delta G / G$ are preferred. In order to enhance the sensitivity, the 'detected' fragmenting quark should have the largest possible transverse momentum. This is then reflected in large $p_{\mathrm{T}(\text { beam) }}$ values for the outgoing hadron(s).

In figure 11 the above-mentioned results on $(\Delta G / G)(x)$ are shown. The three statistically most precise ones are from HERMES on single hadron production on the deuteron at very low $Q^{2}$ [75] and from CoMPAss on high-transverse-momentum hadron-pair production on the deuteron at $Q^{2}<1 \mathrm{GeV}^{2}$ [77] as well as at $Q^{2}>1 \mathrm{GeV}^{2}$ [78]. For the Hermes result, 


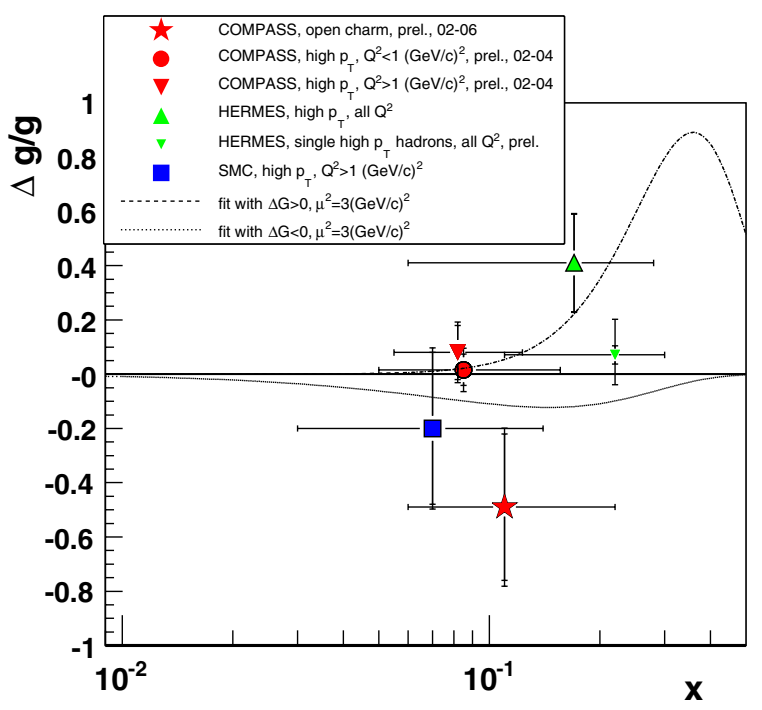

Figure 11. Leading-order results on $(\Delta G / G)(x)$, published or preliminary, from Compass [76-78], Hermes $[75,79]$ and SMC [80]. The sequence of references corresponds to the order of measurements shown in the legend, where also the analysed final state is indicated. Outer error bars, if shown, represent statistical and systematic uncertainties combined in quadrature. Inner error bars represent statistical uncertainties only. Additionally, two equivalently good QCD NLO fits of the structure function $g_{1}^{\mathrm{d}}$ are shown [72], obtained with the constraints $\Delta G<0$ and $\Delta G>0$, respectively. The figure is taken from [76], updated with [78].

very detailed studies were performed to estimate systematic uncertainties associated with Monte Carlo models of the background processes. The major contribution here stems from the uncertainties in the fractional contribution from soft processes, which are poorly known. Among the Compass results on high- $p_{t}$ hadron pairs, the most recent also includes a study of systematic uncertainties associated with the Monte Carlo model.

From the three high- $p_{t}$ hadron results, it can be concluded that $(\Delta G / G)(x)$ at medium $x(0.06-0.3)$ is about 0.1 with a total uncertainty of the same magnitude. This leadingorder result is in fair agreement with the results of NLO fits of $g_{1}$ from [72] shown as curves in figure 11. The value of the unpolarized gluon distribution from the CTEQ6 set of PDFs [81] is $\left.G(x)\right|_{x=0.1} \approx 10$, so that for the gluon helicity distribution $\left.\Delta G(x)\right|_{x=0.1} \approx 1 \pm 1$ is obtained. Expressed as $x \cdot \Delta G(x)$, the LO result from high- $p_{t}$ hadrons is $0.1 \pm 0.1$, in agreement with the less model-dependent result from the NLO QCD fits of [65] discussed in the previous section (see bottom right panel of figure 8). We note that any comparison of NLO to leading-order results has to be done with caution since $k$ factors, defined as their ratio, are known to be large. In the kinematic region considered here, $k$ factors were found [82] of up to 5 for cross sections and up to 2 for asymmetries that are used to extract information on the polarized gluon distribution.

If and when it becomes possible to experimentally constrain the full first moment $\Delta G$ with sufficient accuracy, it will be possible to determine the total orbital angular momentum $\mathcal{L}$ of partons according to (12).

\section{The transverse spin structure of the nucleon}

\subsection{Transverse spin in DIS}

The quark transversity distribution $\delta q_{f}(x)$ was defined in section 1 as the difference between number densities of quarks with transverse polarization in the same and opposite direction as that of the nucleon spin, in a nucleon that is polarized transverse to its 'infinite' momentum. It may at first appear mysterious that it is necessary to distinguish between $\delta q_{f}(x)$ and the helicity distribution $\Delta q_{f}(x)$, since there would be no need to distinguish between them in a non-relativistic system.

All twist-2 parton distributions are defined as light-cone Fourier transforms of products of quark fields $\psi_{f}$ (or gluon fields). The only difference between $q_{f}(x), \Delta q_{f}(x)$ and $\delta q_{f}(x)$ is the Dirac matrices that appear between $\psi_{f}^{\dagger}$ and $\psi_{f}$. For $\Delta q_{f}$ the Dirac matrices are (in Dirac representation, where the lower components vanish in the non-relativistic limit)

$\gamma^{0} \gamma^{i} \gamma^{5}=\left(\begin{array}{cc}1 & 0 \\ 0 & -1\end{array}\right)\left(\begin{array}{cc}0 & \sigma_{i} \\ -\sigma_{i} & 0\end{array}\right)\left(\begin{array}{ll}0 & 1 \\ 1 & 0\end{array}\right)=\left(\begin{array}{cc}\sigma_{i} & 0 \\ 0 & \sigma_{i}\end{array}\right)$

while for $\delta q_{f}$ they read as

$\mathrm{i} \gamma^{0} \sigma^{0 i} \gamma^{5}=\gamma^{i} \gamma^{5}=\left(\begin{array}{cc}0 & \sigma_{i} \\ -\sigma_{i} & 0\end{array}\right)\left(\begin{array}{ll}0 & 1 \\ 1 & 0\end{array}\right)=\left(\begin{array}{cc}\sigma_{i} & 0 \\ 0 & -\sigma_{i}\end{array}\right)$.

So the only difference between $\Delta q_{f}$ and $\delta q_{f}$ is the minus sign in front of the lower components. In the non-relativistic limit, the lower components vanish like $p / m$, so the difference between $\Delta q_{f}$ and $\delta q_{f}$ vanishes like $v^{2} / c^{2}$.

Transversity loses its probabilistic interpretation as a difference in number density distributions when expanded in a helicity basis. Every observable in quantum mechanics has a probabilistic interpretation as the sum of eigenvalues of the observable weighted by the probability to be in each eigenstate $^{6}$. However, if basis functions are used in which the observable is not diagonal, the expectation value of the observable becomes a sum over products of amplitudes and the probabilistic interpretation is 'lost', or at least not apparent. In the present context this elementary principle appears most clearly using Pauli matrices and identifying eigenstates of $\sigma_{z}$ or $\sigma_{x}$ with helicity eigenstates $|\leftrightarrow\rangle$ or transversity eigenstates $|\uparrow\rangle=\frac{1}{\sqrt{2}}\left(|\rightarrow\rangle+\mathrm{e}^{\mathrm{i} \varphi}|\leftarrow\rangle\right)$, respectively, where $\varphi$ specifies the orientation of the transverse polarization direction. Obviously $\sigma_{x}$ is diagonal when expressed in terms of its own eigenvectors, but loses this property when expressed in terms of the eigenvectors of $\sigma_{z}$ :

$\sigma_{x}=|\uparrow\rangle\langle\uparrow|-| \downarrow\rangle\langle\downarrow|=| \leftarrow\rangle\langle\rightarrow|+| \rightarrow\rangle\langle\leftarrow|$.

Therefore the expectation value of the 'transversity operator' $\sigma_{x}$ in some state $\psi$ becomes a sum of products of amplitudes in the helicity basis:

$$
\begin{aligned}
& \left\langle\psi\left|\sigma_{x}\right| \psi\right\rangle=|\langle\psi \mid \uparrow\rangle|^{2}-|\langle\psi \mid \downarrow\rangle|^{2}=\langle\psi \mid \leftarrow\rangle\langle\psi \mid \rightarrow\rangle^{*} \\
& +\langle\psi \mid \rightarrow\rangle\langle\psi \mid \leftarrow\rangle^{*}
\end{aligned}
$$

6 This is one of the postulates of quantum mechanics. 
The right-hand side of (16) is not a sum over absolute squares and hence the probabilistic interpretation for $\left\langle\psi\left|\sigma_{x}\right| \psi\right\rangle$ is no longer apparent.

Despite the loss of a direct probabilistic interpretation, there are (at least) two reasons for discussing transversity in a helicity basis. First, that basis has already been used to study the more familiar longitudinal spin polarization, and secondly only helicity and not transversity is conserved in hard processes. The second statement is based on the observation that for negligible quark masses, both the QCD and QED Lagrangians are invariant under a 'chiral' transformation of the quark spinors:

$$
\psi_{f}(x) \longrightarrow \mathrm{e}^{\mathrm{i} \varepsilon \gamma^{5}} \psi_{f}(x) .
$$

As a consequence of this 'chiral symmetry', the chirality of a massless quark (the eigenvalue of $\gamma^{5}$ ) is conserved. It turns out that for a massless quark its chirality agrees with its helicity, but for an antiquark with the opposite sign ${ }^{7}$. Chiral symmetry thus implies the conservation of the helicity of a massless quark in hard scattering processes. However, even though helicity is conserved, transversity is not, as the scattering amplitudes for the two helicity states may differ in their phase. An analogue from optics is the propagation of light through an optically active medium where the refractive indices for the two circular polarization states are different. While the circular polarization does not change as the beam propagates through this medium, the linear polarization, which is related to the longitudinal polarization through a simple linear combination, precesses around the beam axis since the waves describing the two circular polarization states acquire different phases in such a medium.

An important distinction must be made between transversity and transverse spin. Transverse spin is the expectation value of the operator $\bar{\psi} \gamma^{\perp} \gamma_{5} \psi$ and is chiral-even, whereas chiral-odd transversity is the expectation value of the operator $\bar{\psi} \sigma^{\mu v} \gamma_{5} \psi$, which has matrix elements between states of opposite quark helicity in the proton rest frame. Transverse spin does not have a partonic interpretation in the light-cone framework and is associated with the (twist-3) spin structure function $g_{2}$, whose effects vanish like $1 / \sqrt{Q^{2}}$ in the Bjorken limit, whereas transversity does not vanish in this limit. For a detailed discussion of these subtle issues see [83].

Since hard electromagnetic (or weak) processes conserve the helicity of the quark, inclusive DIS experiments cannot be used to measure the transversity of the quarks. In order to see why, we first use the optical theorem in a transversity basis and then express transversity eigenstates in terms of helicity eigenstates. The difference between the contribution to the cross section from quarks with the same and with opposite transverse polarization as the nucleon would be proportional to the imaginary part of a forward scattering amplitude involving quark helicity flip (see figure 12). However, since absorption of a hard vector boson (e.g. photon) cannot flip the helicity

7 This minus sign for antiquarks also illustrates that, even for massless quarks, chirality and helicity are not the same in the sense of being described by the same operator, which is one reason why it is necessary to introduce the concept of chirality in addition to helicity. Another is that the helicity of a massive particle is frame dependent, whereas chirality is always Lorentz-invariant.

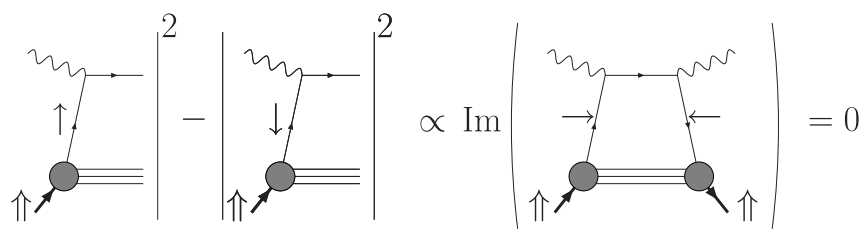

Figure 12. When expressed in terms of quark helicity states, the dependence of the DIS cross section on the transversity of the quarks is proportional to the imaginary part of the quark helicity-flip forward Compton amplitude which vanishes at large $Q^{2}$.

of a massless quark, this forward scattering amplitude must be zero and therefore inclusive DIS cannot be sensitive to the transverse spin of the quarks. Since this argument does not involve the polarization of the photon in DIS, this result also illustrates why a transversely polarized charged lepton beam does not help to determine the transverse polarization of the quarks in inclusive DIS. In semi-inclusive DIS, the meson production vertex can flip quark helicity, which is why the latter process can be sensitive to transverse quark polarization.

It is instructive to compare DIS mediated by vector boson exchange, where quark helicity is conserved, with a gedanken DIS experiment involving scalar bosons, where quark helicity flips at the hard quark-scalar vertex. If only scalar bosons are exchanged, then the quark helicity flips at both vertices in the forward Compton amplitude analogous to figure 12 and such a process would not be sensitive to quark transversity either. Only the interference term between vector and scalar boson exchange provides the single-helicity flip that is needed to make this amplitude non-vanishing, i.e. the interference between photon exchange and the exchange of a hypothetical scalar boson (such as the Higgs boson) would, in principle, be suitable for accessing transversity experimentally using inclusive DIS [84].

\subsection{Tensor charge of the nucleon}

The tensor charge is obtained from the forward matrix element of the operator $\bar{\psi}_{f} \sigma^{\mu \nu} \psi_{f}$, analogously to the vector and axial vector charge (see section 2.2). In terms of quark transversity eigenstates, the tensor charge involves the linear combination $q_{f}^{\uparrow \Uparrow}-q_{f}^{\downarrow \Uparrow}-\left(\bar{q}_{f}^{\uparrow \Uparrow}-\bar{q}_{f}^{\downarrow \Uparrow}\right)$, i.e. the transversity of the quarks minus the transversity of the antiquarks. The fact that the tensor charge contains the difference between quark and antiquark transversity is convenient as the contributions from shortlived virtual $q \bar{q}$ pairs cancel, and it can be identified with the transversity of valence quarks only. Moreover, the tensor charge can be computed in lattice gauge theory, and with better precision than the axial charge (see section 4.4).

In a helicity basis, transversity involves both a quark and a nucleon helicity flip, i.e. it involves the overlap between a nucleon 'state' that had positive helicity and from which a quark with positive helicity has been removed and another nucleon 'state' that had negative helicity and from which a quark with negative helicity has been removed. The CauchySchwarz inequality tells us that this 'scalar product' is less than the norms of the states involved. Since the latter represent the probability to find a quark with the same helicity as the nucleon 
spin, one thus finds the Soffer inequality [85]

$$
2\left|\delta q_{f}(x)\right| \leqslant 2 q_{f}^{\vec{\Rightarrow}}(x)=q_{f}(x)+\Delta q_{f}(x),
$$

which holds independently for each quark and antiquark flavour. It provides useful constraints on parametrizations of transversity.

Attempts have been made to develop a 'transversity sum rule' $[86,87]$ that is analogous to the 'Ji decomposition' of the nucleon spin (39) but involves the net transversity of quarks (plus antiquarks). However, one problem with any transversity sum rule is that quarks and antiquarks contribute with opposite signs to the tensor charge (first moment of transversity), while they contribute to either longitudinal or transverse nucleon spin with the same sign. Therefore, transversity cannot contribute to such a sum rule in terms of the tensor charge, but rather in terms of the tensor charge for the quarks plus the antiquarks - a combination that cannot be represented in terms of a local operator. Therefore lattice calculations will be unable to calculate the transversity contribution to a transversity sum rule. Furthermore, in the transversity sum rule in [86, 87], neither an operator definition nor an experimental procedure for determining the orbital angular momentum contribution has been provided. Thus this sum rule cannot be tested and is unfalsifiable.

\subsection{Transversity in the Drell-Yan (DY) reaction}

The conceptually simplest practical way to measure transversity is to exploit the DY reaction in doubly transversely polarized hadron-hadron collisions $\mathrm{h}_{1}^{\Uparrow(\Downarrow)}+\mathrm{h}_{2}^{\Uparrow(\Downarrow)} \longrightarrow \mu^{+} \mu^{-}+X$, involving the hard scattering process

$$
q+\bar{q} \longrightarrow \gamma^{*} \longrightarrow \mu^{+} \mu^{-} .
$$

The high-energy cross section for this reaction is obtained by convoluting the quark distribution in one of the hadrons with the antiquark distribution in the other hadron and with the cross section for $q+\bar{q} \longrightarrow \mu^{+} \mu^{-}$, where the latter can be calculated in perturbation theory (one photon in the intermediate state plus higher order corrections). Transversity can be studied in DY experiments with transversely polarized (anti-) protons because the quark and antiquark preferentially annihilate into a vector boson when their transverse spins are parallel. Hence the double-spin asymmetry $A_{\mathrm{TT}}$ is capable of measuring the correlation between the spin of the quark in one of the hadrons with that of the antiquark in the other hadron. This doublespin asymmetry is therefore sensitive to the product of the transversity distributions for quarks and antiquarks carrying momentum fractions $x_{1}$ and $x_{2}$, respectively:

$A_{\mathrm{TT}}\left(x_{1}, x_{2}\right)=\frac{\sigma \Uparrow \Uparrow-\sigma \Uparrow \Downarrow}{\sigma \Uparrow \Uparrow+\sigma \Uparrow \Downarrow} \propto \sum_{f} e_{f}^{2} \delta q_{f / \mathrm{h}_{1}}\left(x_{1}\right) \delta \bar{q}_{f / \mathrm{h}_{2}}\left(x_{2}\right)$.

The momentum fractions of the quark and antiquark can be reconstructed from the invariant mass and net longitudinal momentum of the muon pair into which they annihilate. The struck $\bar{q}$ in a DY reaction can be either a sea or valence quark in an incident antiproton (or meson or proton). In the interpretation of the data for $\mathrm{p}+\mathrm{p} \longrightarrow \mu^{+} \mu^{-}+X$, one $x$ value can be associated with the $\bar{q}$ by considering asymmetric events where the momentum fraction of one of the initial quarks was so high that it is very unlikely to have been an antiquark since the latter carry only small momentum fractions. As the DY cross section depends on products of PDFs, determining the overall normalization may in general be ambiguous. In the case of $\overline{\mathrm{p}}+\mathrm{p} \longrightarrow \mu^{+} \mu^{-}+X$ there is no such ambiguity as the same valence PDF of the proton appears as a product with itself. Once this normalization has been determined, it can be used to determine the normalization of sea quarks in $p p$ scattering. Plans are being laid at several laboratories to exploit this reaction to measure transversity [88, 89].

All existing experimental constraints on transversity are based on the sensitivity of fragmentation to the transverse polarization of the struck quark in processes other than DY, as manifested in single-spin asymmetries. These will be discussed in section 3.5.

\subsection{Intrinsic parton transverse momentum}

Partons can also have momentum $\boldsymbol{p}_{\mathrm{T}}$ transverse to the infinite momentum of the hadron. Hence parton distributions can be introduced that depend not only on $x$ but also on $p_{\mathrm{T}}$ $[90,91]$ and have interpretations as number densities or differences between number densities [92]. Only three of these distributions survive integration over $\boldsymbol{p}_{\mathrm{T}}$ leading to the familiar PDFs that depend only on $x$. These have already been discussed in section 1 . They are the spin-averaged distribution $q_{f}\left(x, \boldsymbol{p}_{\mathrm{T}}\right)$, the helicity distribution $\Delta q_{f}\left(x, \boldsymbol{p}_{\mathrm{T}}\right)$ and the transversity distribution $\delta q_{f}\left(x, p_{\mathrm{T}}\right)$. However, many other twist-2 distributions exist and they have probabilistic interpretations similar to that of integrated PDFs [92]. These distributions ${ }^{8}$ are sometimes known as transverse-momentumdependent (TMD) parton distributions (TMD-PDFs, or simply TMDs). In the rest of this subsection we take the unpolarized case as an example.

When a quark in a nucleon absorbs a hard virtual photon, the resulting momentum of the quark is the sum of its initial momentum and the momentum transferred from the lepton. In leading order $\alpha_{s}$, any momentum component transverse to the virtual photon direction thus originates from the intrinsic transverse momentum $\boldsymbol{p}_{\mathrm{T}}$ of the parton before the scattering, described by $q_{f}\left(x, \boldsymbol{p}_{\mathrm{T}}\right)$.

The distribution of hadrons $h$ in a jet originating from a fast quark of flavour $f$ is described by fragmentation functions $D_{f / \mathrm{h}}\left(z, \boldsymbol{k}_{\mathrm{T}}\right)$. Here $\boldsymbol{k}_{\mathrm{T}}=\boldsymbol{p}_{\mathrm{h}, \mathrm{T}}-z \boldsymbol{p}_{\mathrm{T}}$ is the transverse momentum of the hadron relative to the momentum of the fragmenting quark. In DIS, the factorized semi-inclusive cross section for producing a hadron with transverse momentum $p_{\mathrm{h}, \mathrm{T}}$ with respect to the virtual-photon momentum is obtained by convoluting ${ }^{9}$ the transverse-momentum-dependent parton distributions $q_{f}\left(x, \boldsymbol{p}_{\mathrm{T}}\right)$ with these fragmentation functions:

$$
\begin{aligned}
& \sigma^{\mathrm{ep} \rightarrow \mathrm{eh} X}\left(x_{\mathrm{B}}, z, \boldsymbol{p}_{\mathrm{h}, \mathrm{T}}\right) \propto \sum_{f} q_{f}\left(x_{\mathrm{B}}, \boldsymbol{p}_{\mathrm{T}}\right) \otimes D_{f / \mathrm{h}}\left(z, \boldsymbol{k}_{\mathrm{T}}\right) \\
& \quad \otimes \delta\left(z \boldsymbol{p}_{\mathrm{T}}+\boldsymbol{k}_{\mathrm{T}}-\boldsymbol{p}_{\mathrm{h}, \mathrm{T}}\right) .
\end{aligned}
$$

8 In the 'Amsterdam notation', distributions that do not 'survive' integration over $\mathrm{d}^{2} \boldsymbol{p}_{\mathrm{T}}$ are signified by the superscript $\perp$.

9 Only for Gaussian dependences can these convolutions be factorized. 


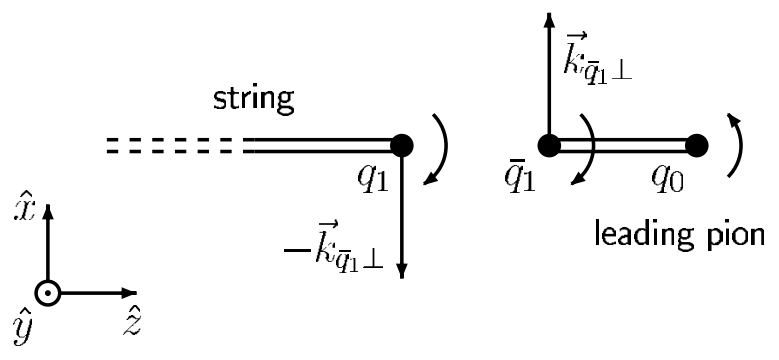

Figure 13. Behind the leading quark $\left(q_{0}\right)$ moving along $\hat{z}$ a string forms, which eventually breaks through the formation of the $\bar{q}_{1} q_{1}$ pair. In the ${ }^{3} \mathrm{P}_{0}$ model, the $\bar{q} q$ pair has orbital angular momentum in the opposite direction as the spin of the $\bar{q} q$ pair, i.e. in the case of fragmentation into pions ( spin 0 ) in the same direction as the spin of the leading quark. Since the antiquark from the pair is expected to be produced closer to the leading quark as the produced quark, this implies that the produced antiquark inherits a positive transverse momentum $\vec{k}_{\bar{q}_{1} \perp}$ in the $\hat{y}$-direction from the orbital motion (for a leading quark polarized in the $\hat{y}$-direction).

These fragmentation functions are expected to be universal, i.e. independent of both process and target [93]. For example, the distribution of hadrons in jets produced in $\mathrm{e}^{+} \mathrm{e}^{-} \rightarrow$ hadrons is described by the same fragmentation functions that describe jets in DIS. However, as discussed in section 3.6, initial state interactions (ISIs) and FSIs need to be considered before the concept of universality can be literally applied.

\subsection{Semi-inclusive DIS and the Collins effect}

When a transversely polarized quark fragments into hadrons, the structure of the jet is sensitive to the polarization of the quark. In particular, the orientation of the transverse spin direction together with the quark's momentum defines a plane relative to which the momentum distribution of hadrons may not be left-right symmetric. To understand how this may occur, we consider a simple model for the case of fragmentation into pions: in this process for example a quark with spin pointing up needs to pick up from the vacuum an antiquark with spin down in order to form a spin zero state. The ${ }^{3} \mathrm{P}_{0}$ model for quark-anti-quark pair creation [94] postulates that the $q \bar{q}$ pair is created with 'vacuum quantum numbers' $J^{P}=0^{+}$, the simplest configuration of which is $S=1, L=1$ and no gluon. This model is based on the idea that as the QCD string between the fragmenting quark and the target remnant is stretched and stores energy, a $q \bar{q}$ pair can tunnel from the vacuum to remove some of the stored energy and the string breaks. Due to angular momentum conservation, this model suggests that the $q \bar{q}$ pair is thus produced with orbital angular momentum in the same direction (i.e. up in this case) as the spin of the fragmenting quark in the produced pion (see figure 13). The antiquark that merges with the fragmenting quark should retain some of that transverse orbital angular momentum, causing it to move to the left (when looking in the direction of motion of the fragmenting quark with spin up) [95]. The pion inherits the transverse momentum carried by the antiquark.

The fragmentation function $H_{1, f / \mathrm{h}}^{\perp}\left(z, k_{\mathrm{T}}\right)$ describing the left-right asymmetry in the fragmentation of a transversely polarized quark of flavour $f$ into a hadron $\mathrm{h}$ was identified by Collins [96], and found to be chirally odd. Its combination

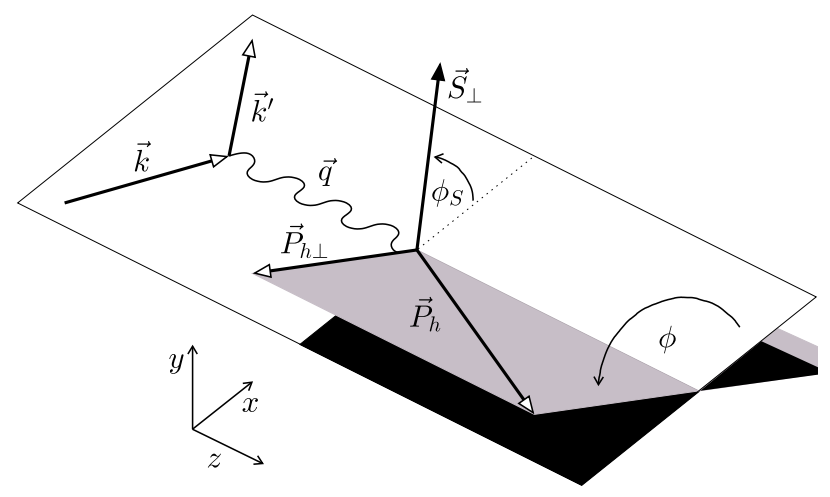

Figure 14. The definitions of the azimuthal angles of the hadron production plane and the axis of the relevant component $\vec{S}_{\perp}$ of the target spin, relative to the scattering plane that contains the momenta $\vec{k}$ and $\vec{k}^{\prime}$ of the incident and scattered leptons.

with transversity $\delta q_{f}(x)$ that describes the distribution of transversely polarized quarks thus gives rise to a correlation between the transverse target spin and the transverse momentum of the produced hadron(s). The associated asymmetry is proportional to $\sin \left(\phi-\phi_{q}\right)$, where $\phi_{q}$ is the azimuthal angle of the polarization vector of the fragmenting quark about the virtual photon direction and $\phi$ is the azimuthal angle of the trajectory of the produced hadron h. However, the polarization direction of the struck quark and that of the fragmenting quark are not identical. In QED, when the electron emits a high-momentum virtual photon, the electron helicity is conserved and therefore the orientation of the electron spin is rotated in the lepton scattering plane. The virtual photon inherits the change in angular momentum and is thus linearly polarized in the lepton scattering plane. When a quark absorbs this linearly polarized photon, its spin component along the polarization direction of the photon tends to flip. For a transversely polarized quark, this implies that its polarization direction is on average tilted symmetrically with respect to the normal of the lepton scattering plane. For azimuthal angles measured relative to the lepton scattering plane, as illustrated in figure 14, the tilt results in $\phi_{q}=\pi-\phi_{S}$, where $\phi_{S}$ denotes the polarization direction of the target nucleon, since the polarization of the quark before the photoabsorption is proportional to the product of the target nucleon polarization and the transversity distribution. The resulting $\sin \left(\phi+\phi_{S}\right)$ angular dependence can thus be used to extract the product of transversity and the Collins fragmentation function.

However, this 'Collins effect' is not the only mechanism that can give rise to a correlation between the target spin and the momenta of final state hadrons. A correlation between transverse momenta of quarks in the target and the target nucleon spin may give rise to a $\sin \left(\phi-\phi_{S}\right)$ asymmetry, as we will now discuss.

\subsection{The Sivers effect}

In a target that is polarized transverse to the virtual-photon direction (e.g. up), the quarks in the target nucleon can exhibit a (left/right) asymmetry of the distribution $f_{q / \mathrm{p}^{\uparrow}}\left(x, \boldsymbol{p}_{\mathrm{T}}\right)$ in their 
transverse momentum $\boldsymbol{p}_{\mathrm{T}}$

$f_{q / \mathrm{p}^{\uparrow}}\left(x, \boldsymbol{p}_{\mathrm{T}}\right)=f_{1}^{q}\left(x, p_{\mathrm{T}}^{2}\right)-f_{1 \mathrm{~T}}^{\perp q}\left(x, p_{\mathrm{T}}^{2}\right) \frac{\left(\hat{\boldsymbol{P}} \times \boldsymbol{p}_{\mathrm{T}}\right) \cdot \boldsymbol{S}}{M}$,

where $\boldsymbol{S}$ is the spin of the target nucleon and $\hat{\boldsymbol{P}}$ is a unit vector opposite to the direction of the virtual-photon momentum. The fact that such a term may be present in (22) is known as the Sivers effect and the function $f_{1 \mathrm{~T}}^{\perp q}\left(x, p_{\mathrm{T}}^{2}\right)$ is known as the Sivers function [97]. The latter vanishes in a naive parton picture since $\left(\hat{\boldsymbol{P}} \times \boldsymbol{p}_{\mathrm{T}}\right) \cdot \boldsymbol{S}$ is odd under naive time reversal (a property known as naive-T-odd), where one merely reverses the direction of all momenta and spins without interchanging the initial and final states. In fact, until a few years ago, it was believed that full time reversal invariance of QCD forbids the existence of the Sivers effect. However, then it was realized that a FSI mediated by soft gluon(s) between the struck quark and the rest of the target nucleon, before the quark fragments into hadrons, could avoid this restriction [98]. This led to a generalization of our understanding of what is meant by a parton distribution and its universality, and how it may appear in expressions for matrix elements of processes. The momentum fraction $x$, which is equal to $x_{\mathrm{B}}$ in DIS experiments, represents the longitudinal momentum of the quark before it absorbs the virtual photon, as it is determined solely from the kinematic properties of the virtual photon and the target nucleon. In this new view, in DIS the transverse momentum $\boldsymbol{p}_{\mathrm{T}}$ represents the asymptotic transverse momentum of the active quark after it has absorbed the virtual photon and then left the target but still before it fragments into hadrons. Thus the Sivers function for semiinclusive DIS includes the FSI between struck quark and target remnant, and time reversal invariance no longer requires that it vanishes. Indeed, time reversal not only reverses the signs of all spins and momenta, but also transforms FSIs into ISIs. It has been shown that the Sivers function relevant for SIDIS and that relevant for DY processes must have opposite signs [98]:

$$
f_{1 \mathrm{~T}}^{\perp}\left(x, p_{\mathrm{T}}^{2}\right)_{\mathrm{SIDIS}}=-f_{1 \mathrm{~T}}^{\perp}\left(x, p_{\mathrm{T}}^{2}\right)_{\mathrm{DY}},
$$

where the asymmetry in DY arises from the ISI between the incoming antiquark and the target. The experimental verification of this relation would provide a test of the current understanding of the Sivers effect within QCD. While the argument leading to (23) is non-perturbative, it is instructive to elucidate its physical origin in the context of a perturbative picture: for instance, when the virtual photon in a DIS process hits a red quark, the spectators must be collectively anti-red in order to form a colour-neutral bound state, and thus attract the struck quark. In DY, when an anti-red antiquark annihilates with a target quark, the target quark must be red in order to merge into a photon, which carries no colour. Since the proton was colourless before the scattering, the spectators must be anti-red and thus repel the approaching antiquark [99].

Another reason why the Sivers effect is attracting attention is that it relates to the correlation between the orbital angular momentum of partons and the spin of the parent nucleon. A semi-classical argument can help to understand how. Should quarks have orbital angular momentum, it would be correlated with the spin of the nucleon, as the latter defines the only
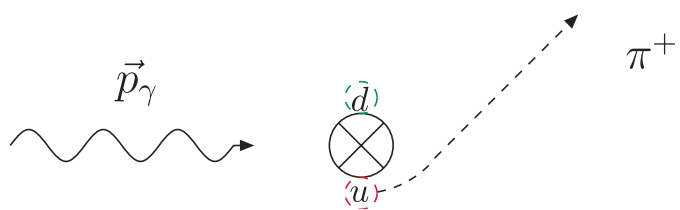

Figure 15. A correlation between the nucleon spin and the quark orbital angular momentum, in combination with an attractive FSI, gives rise to a Sivers effect for $u(d)$ quarks imparting a transverse momentum that is on average directed upward (downward).

preferred direction. When viewed in the nucleon rest frame, quarks orbiting in a transversely polarized nucleon move towards the virtual photon on one side of the nucleon but away from it on the opposite side. Upon boosting the nucleon to the infinite-momentum frame, the quarks on one side of the nucleon with a particular momentum fraction $x$ thus appear to have larger longitudinal momenta than on the other side. Since parton distributions are rapidly falling functions of $x$, shifting them towards larger (smaller) $x$ results in an increased (decreased) probability of interaction when viewed at a fixed value of $x$. The result is that, at a given value of $x$, more quarks absorb photons on the left or right side of the nucleon when the nucleon spin is pointing e.g. up. As the struck quark moves away, it experiences an attractive FSI that tends to 'focus' it towards the axis through the centre of the nucleon along the direction of the virtual photon (see figure 15). Thus the excess of interactions on one side results in a tendency of the struck quark and hence the hadron jet to be deflected towards the opposite side, producing a sinusoidal azimuthal distribution of the hadrons relative to the transverse polarization direction of the target nucleon. Quarks of different flavours may have different spin-orbit correlations, and also tend to produce different populations of hadron types. Hence different hadron types may have different azimuthal distributions.

The type of deflection shown in figure 15 also arises in optics when a convex lens is illuminated asymmetrically, e.g. when only one side of the lens is illuminated with a laser beam parallel to the axis of the lens. The beam is then deflected towards the lens axis and the outgoing beam has a left-right 'momentum asymmetry' [100]. Because of this analogy, the above mechanism has been called 'chromodynamic lensing' [101].

As the asymmetry is caused by the orbital motion of the quarks, it is a (left-right) asymmetry relative to the direction of the (vertical) transverse target polarization, i.e. proportional to $\sin \left(\phi-\phi_{S}\right)$ in SIDIS. This differs from the $\sin \left(\phi+\phi_{S}\right)$ angular dependence caused by the Collins effect, thus permitting the two contributions to be separated through the use of transverse target polarization.

The Sivers function not only provides a clue about the orbital angular momentum structure of the nucleon wave function, but in addition carries important information about the complex phase structure of the FSI [102].

\subsection{Boer-Mulders effect}

Even when the target nucleon is unpolarized, there can exist a correlation between the distribution in transverse momentum 


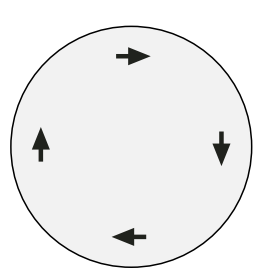

(a)

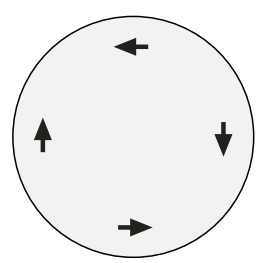

(d)

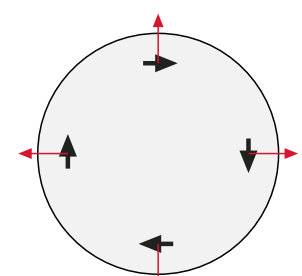

(b)

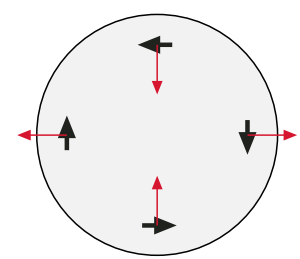

(e)

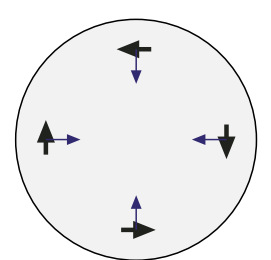

(c)

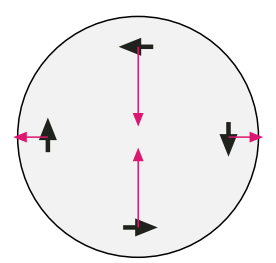

(f)
Figure 16. Illustration for the interplay between transverse momenta from the Boer-Mulders and the Collins effect, leading to a $\cos 2 \phi$ asymmetry: (a) 'primordial' transverse spin distribution in an unpolarized target, $(b)$ transverse momentum from the Collins effect for hypothetical SIDIS without transverse spin flip, $(c)$ transverse momentum from the attractive FSI, $(d)$ after the virtual photon absorption, the transverse spin component in the (horizontal) lepton scattering plane has flipped, $(e)$ transverse momentum from the Collins effect including the transverse spin flip, $(f)$ net transverse momentum from the FSI and the Collins effect.

and transverse polarization of its quarks. The Boer-Mulders effect is a possible dependence of the distribution $f_{q^{\uparrow} / \mathrm{p}}\left(x, \boldsymbol{p}_{\mathrm{T}}\right)$ of transversely polarized quarks in an unpolarized target on the transverse polarization $\vec{S}_{q}$ of the quarks:

$f_{q^{\uparrow} / \mathrm{p}}\left(x, \boldsymbol{p}_{\mathrm{T}}\right)=\frac{1}{2}\left[f_{1}^{q}\left(x, p_{\mathrm{T}}^{2}\right)-h_{1}^{\perp q}\left(x, p_{\mathrm{T}}^{2}\right) \frac{\left(\hat{\boldsymbol{P}} \times \boldsymbol{p}_{\mathrm{T}}\right) \cdot \boldsymbol{S}_{q}}{M}\right]$,

and the Boer-Mulders function $h_{1}^{\perp q}\left(x, p_{\mathrm{T}}^{2}\right)$ embodies this correlation [103], which can naturally arise from nonperturbative spin-orbit interactions.

In the case of the Sivers effect, the relevant spin is that of the target nucleon, whereas here it is that of the struck quark. However, both effects can be understood to arise in part from the preference of the virtual photon to absorb on a quark with excess momentum fraction associated with its orbital motion, because of the monotonic decrease with $x$ in the unpolarized PDF. Thus a quark found on the top (bottom) [left] \{right side of the nucleon, as seen by the virtual photon, will tend to have orbital angular momentum pointing right (left) [up] \{down\} (on first reading it may be helpful to consider only the first option and compare with figure 16). If the quark spin is correlated with its orbital angular momentum as represented by the Boer-Mulders function, then its polarization will have a similar (or opposite) spatial pattern (figure 16(a)). As described in section 3.5, the quark polarization affects the transverse momentum of the produced hadrons via the Collins effect. Quarks with polarization pointing right (left) [up] \{down\} may be deflected, e.g., up (down) [left] \{right\}, or the opposite depending on the sign of the Collins fragmentation function. We first consider the simpler case of exchange of a scalar boson rather than the vector photon, where the former cannot carry any information about the orientation of the lepton scattering plane so that the initial quark transverse polarization direction persists through the photoabsorption process. Then the combined effect would be a net 'focusing' or 'defocusing' of the produced hadrons relative to the direction of the exchanged boson, according to the product of the Collins function with the Boer-Mulders function representing the original spin-orbit correlation of the quark (figure 16(b)). However, we must beware that this term '(de)focusing' can be misleading in this context. Since one side of the nucleon is not experimentally distinguished from the other, and the target nucleon is uniformly illuminated by the parallel 'beam' of virtual photons, the effect only randomly scatters or smears the direction of the hadron. At each azimuthal angle of the hadron direction about the virtual photon direction there is a net smearing effect, while the mean change of direction due to this '(de)focusing' is zero.

An additional independent axially symmetric 'focusing' action is provided by the attractive FSI that is crucial also for the Sivers effect (figure 16(c)). (The Sivers effect itself plays no role here because the target nucleon is unpolarized.) This FSI effect either enhances or competes with that in figure $16(b)$ to produce a net '(de)focusing' action on the direction of the produced meson relative to that of the virtual photon. Thus for scalar exchange, the net effect may be a change in the cross section for hadron production that is independent of azimuth. For example, for observed values of the meson momentum component $p_{\mathrm{h}, \mathrm{T}}$ transverse to the direction of the virtual photon that are larger than the most probable value, this smearing would increase the cross section.

On the other hand, as also described in section 3.5, for vector (photon) exchange, the initial transverse quark polarization does not always persist through the photoabsorption process. Because of the linear polarization of the virtual photon in the lepton scattering plane, the component of the quark polarization in that plane can be flipped (figure $16(d)$ ), inverting the effect in figure $16(b)$ only for hadron transverse momentum components normal to that plane (figure 16(e)). Now this effect tends to enhance the FSI effect in one plane and cancel it in the orthogonal plane (figure $16(f)$ ). Since the net smearing effect is proportional to the magnitude of the net '(de)focusing' action, the smearing is different in the lepton scattering plane from that in the orthogonal plane. Hence the effect on the cross section can be different for hadrons produced in the lepton scattering plane compared with the orthogonal plane. This amounts to a $\cos (2 \phi)$ dependence of the hadron azimuthal distribution, relative to the lepton scattering plane. In the absence of the FSI, the inversion of the Collins effect in the lepton scattering plane (illustrated in figure 16(e)) would not change the '(de)focusing' magnitude, which is all that matters for the cross section. The necessary role of the FSI is associated with the T-odd nature of this Boer-Mulders effect.

A more formal argument is also included here for the sake of completeness, as it may not be easily available in the literature. The Collins effect acts as a polarimeter with a analysing power $\propto H_{1}^{\perp}\left(x, k_{\mathrm{T}}\right)\left(\hat{\boldsymbol{P}} \times \boldsymbol{k}_{\mathrm{T}}\right) \cdot \boldsymbol{S}_{q}^{\prime}$, which tags the transverse polarization $S_{q}^{\prime}$ of the quark after it has absorbed the virtual photon and its component in the lepton 
scattering plane has flipped compared with $\boldsymbol{S}_{q}$. Here $\boldsymbol{k}_{\mathrm{T}}$ is the transverse momentum of the outgoing hadron relative to the momentum direction of the fragmenting quark. The resulting asymmetry in the hadron distribution relative to the lepton scattering plane is obtained by convoluting the Boer-Mulders function with the Collins function to obtain the distribution of the transverse (with respect to the virtual photon direction) hadron momentum $p_{\mathrm{h}, \mathrm{T}}$ in the final state. (For simplicity, the dependence of these functions on the longitudinal momentum is not shown here.) In addition, the result must be averaged over the (unmeasured) transverse polarization direction $\phi_{S}$ of the quark in the initial state:

$$
\begin{gathered}
A\left(\boldsymbol{p}_{\mathrm{h}, \mathrm{T}}\right) \propto \int_{0}^{2 \pi} \mathrm{d} \phi_{S} \int \mathrm{d}^{2} \boldsymbol{p}_{\mathrm{T}} \int \mathrm{d}^{2} \boldsymbol{k}_{\mathrm{T}} h_{1}^{\perp q}\left(p_{\mathrm{T}}^{2}\right)\left(\boldsymbol{p}_{\mathrm{T}} \times \boldsymbol{S}_{q}\right)_{z} \\
\times H_{1}^{\perp}\left(k_{\mathrm{T}}^{2}\right)\left(\boldsymbol{k}_{\mathrm{T}} \times \boldsymbol{S}_{q}^{\prime}\right)_{z} \delta\left(\boldsymbol{p}_{\mathrm{T}}+\boldsymbol{k}_{\mathrm{T}}-\boldsymbol{p}_{\mathrm{h}, \mathrm{T}}\right) .
\end{gathered}
$$

As $p_{\mathrm{h}, \mathrm{T}}$ is the only vector on which the transverse momentum integral can depend, rotational invariance implies

$$
\begin{gathered}
\int \mathrm{d}^{2} \boldsymbol{p}_{\mathrm{T}} \int \mathrm{d}^{2} \boldsymbol{k}_{\mathrm{T}} h_{1}^{\perp q}\left(p_{\mathrm{T}}^{2}\right) k_{\mathrm{T}, i} H_{1}^{\perp}\left(k_{\mathrm{T}}^{2}\right) p_{\mathrm{T}, j} \delta\left(\boldsymbol{p}_{\mathrm{T}}+\boldsymbol{k}_{\mathrm{T}}-\boldsymbol{p}_{\mathrm{h}, \mathrm{T}}\right) \\
=a\left(p_{\mathrm{h}, \mathrm{T}}^{2}\right) \delta_{i j}+b\left(p_{\mathrm{h}, \mathrm{T}}^{2}\right) p_{\mathrm{h}, \mathrm{T}}^{i} p_{\mathrm{h}, \mathrm{T}}^{j},
\end{gathered}
$$

with some suitable functions $a\left(p_{\mathrm{h}, \mathrm{T}}^{2}\right)$ and $b\left(p_{\mathrm{h}, \mathrm{T}}^{2}\right)$, yielding

$$
\begin{aligned}
& A\left(\boldsymbol{p}_{\mathrm{h}, \mathrm{T}}\right) \propto \int_{0}^{2 \pi} \mathrm{d} \phi_{S}\left[a\left(p_{\mathrm{h}, \mathrm{T}}^{2}\right) \boldsymbol{S}_{q} \cdot \boldsymbol{S}_{q}^{\prime}\right. \\
& \left.\quad+b\left(p_{\mathrm{h}, \mathrm{T}}^{2}\right)\left(\boldsymbol{S}_{q} \times \boldsymbol{p}_{\mathrm{h}, \mathrm{T}}\right)_{z}\left(\boldsymbol{S}_{q}^{\prime} \times \boldsymbol{p}_{\mathrm{h}, \mathrm{T}}\right)_{z}\right] .
\end{aligned}
$$

The transverse polarization of the active quark does not always flip, but events where it does not flip do not produce any asymmetry. For the determination of the asymmetry we thus focus on those events where the transverse spin component in the lepton scattering plane flips. Taking this plane to lie in the $\hat{x}-\hat{z}$ plane, the transverse polarization vectors take on the form $S_{q}=\left(\cos \phi_{S}, \sin \phi_{S}\right)$ and $S_{q}^{\prime}=\left(-\cos \phi_{S}, \sin \phi_{S}\right)$, yielding

$$
\begin{aligned}
& \int_{0}^{2 \pi} \mathrm{d} \phi_{S} \boldsymbol{S}_{q} \cdot \boldsymbol{S}_{q}^{\prime}=0 \\
& \int_{0}^{2 \pi} \mathrm{d} \phi_{S}\left(\boldsymbol{S}_{q} \times \boldsymbol{p}_{\mathrm{h}, \mathrm{T}}\right)_{z}\left(\boldsymbol{S}_{q}^{\prime} \times \boldsymbol{p}_{\mathrm{h}, \mathrm{T}}\right)_{z}=\frac{\pi}{2}\left(p_{\mathrm{h}, \mathrm{T}, x}^{2}-p_{\mathrm{h}, \mathrm{T}, y}^{2}\right) \\
& \quad=\frac{\pi}{2} p_{\mathrm{h}, \mathrm{T}}^{2} \cos 2 \phi
\end{aligned}
$$

where $\phi$ specifies the transverse direction of the outgoing hadron momentum $\boldsymbol{p}_{\mathrm{h}, \mathrm{T}}$. In combination with (26) this implies a $\cos 2 \phi$ asymmetry for the transverse momentum distribution of the produced hadrons:

$$
A\left(\boldsymbol{p}_{\mathrm{h}, \mathrm{T}}\right) \propto p_{\mathrm{h}, \mathrm{T}}^{2} b\left(p_{\mathrm{h}, \mathrm{T}}^{2}\right) \cos 2 \phi,
$$

which involves a convolution $b\left(p_{\mathrm{h}, \mathrm{T}}^{2}\right)$ of the Boer-Mulders and Collins functions. We note here that a flip of the quark polarization direction relative to the normal of the lepton scattering plane is essential to provide a reference plane (the lepton scattering plane) for the transverse momentum of the outgoing hadrons and thus making the Boer-Mulders effect observable, just as it was essential for separating the Collins from the Sivers effect in SIDIS. We also note that the BoerMulders effect is a spin effect, even though no spin is being measured.

Alternatively, one can also study the $\cos 2 \phi$ asymmetry in the unpolarized DY process, in which case $\phi$ is the azimuthal angle of the $\mu^{+} \mu^{-}$plane about the virtual photon axis with respect to the incident proton trajectory.

While ISI/FSI are obviously essential features for the Sivers and Boer-Mulders functions, they affect all twist-2 $p_{\mathrm{T}}$-dependent parton distributions. However, this does not spoil standard universality, as all other $\boldsymbol{p}_{\mathrm{T}}$-dependent PDFs are (naive) T-even and thus the ISI in DY has the same effect as the FSI in SIDIS. Nevertheless, their $\boldsymbol{p}_{\mathrm{T}}$ dependence is expected to differ from the 'intrinsic' $\boldsymbol{p}_{\mathrm{T}}$ dependence that one would for example obtain theoretically through the evaluation of a matrix element of a local operator, as is usually done in lattice gauge theory (see section 4.4).

\subsection{Recent semi-inclusive data with transverse target polarization}

Almost 20 years ago, surprisingly large single-spin asymmetries were observed in inclusive production of pions by beams of polarized protons and anti-protons [104]. (Recently this phenomenon was also observed at the higher energies of RHIC [105, 106], where the cross section for these reactions can be understood within the framework of perturbative QCD.) Various theoretical interpretations were proposed, including the Collins and Sivers effects. However, no way of disentangling these effects on those data could be found. A similar ambiguity plagued the interpretation of the first substantial single-spin asymmetries, with respect to longitudinal target polarization, observed in the semi-inclusive leptoproduction of mesons by the Hermes experiment [107]. When the target is polarized along the lepton beam axis, both the Collins and Sivers mechanisms produce a common sinusoidal behaviour in the azimuthal angle $\phi$ of the detected hadron about the direction of the virtual photon, with respect to the lepton scattering plane. On the other hand, when the target polarization is orthogonal to the beam axis (more precisely, when it is orthogonal to the direction of the virtual photon), the azimuthal angle $\phi_{S}$ of the axis of transverse target polarization resolves the effects of the two mechanisms into distinctive signatures: $\sin \left(\phi-\phi_{S}\right)$ for the Sivers mechanism and $\sin \left(\phi+\phi_{S}\right)$ for the Collins mechanism [103]. In DIS, the Collins effect serves as a 'polarimeter' sensitive to transverse quark polarization to reveal its correlation with transverse target polarization, which is known as transversity. As described in section 3.4, the Collins mechanism depends on the influence of the struck quark's polarization on the transverse momentum $\boldsymbol{k}_{\mathrm{T}}$ acquired by the produced hadron in the fragmentation process, in particular on the component of $\boldsymbol{k}_{\mathrm{T}}$ that is orthogonal to the quark's transverse polarization. This 'measurement' of the struck quark's polarization occurs after its spin component in the lepton scattering plane has been flipped by the photoabsorption. The orientation of the lepton scattering plane is 'remembered' by the virtual photon because that is the plane of its linear polarization. Hence 
the Collins mechanism is sensitive to the orientation of the lepton scattering plane from which the azimuthal angles are measured, as indicated by the dependence on $\phi+\phi_{S}$. In contrast, as described in section 3.6, the Sivers effect can be understood in terms of retention by the struck quark of the $p_{\mathrm{T}}$ that it had in the target due to a correlation with transverse target polarization. This transverse momentum tends to be inherited by a forward hadron that may 'contain' this quark. Hence in either case, the hadron $p_{\mathrm{h}, \mathrm{T}}$ is correlated with transverse target polarization. However, the different azimuthal dependences allow their experimental separation, and semi-inclusive DIS measurements with a transversely polarized target were eagerly anticipated.

The first such data were reported a few years ago by Hermes [114]. The cross section asymmetry with respect to target polarization was extracted as a two-dimensional distribution in $\phi$ versus $\phi_{S}$. The Collins azimuthal moment $\left\langle\sin \left(\phi+\phi_{S}\right)\right\rangle_{\mathrm{UT}}^{\mathrm{h}}$ and the Sivers moment $\left\langle\sin \left(\phi-\phi_{S}\right)\right\rangle_{\mathrm{UT}}^{\mathrm{h}}$ of the virtual-photon asymmetry were extracted in the fit

$$
\begin{aligned}
& \frac{A_{\mathrm{UT}}^{\mathrm{h}}\left(\phi, \phi_{S}\right)}{2}=\left\langle\sin \left(\phi+\phi_{S}\right)\right\rangle_{\mathrm{UT}}^{\mathrm{h}} \sin \left(\phi+\phi_{S}\right) \\
& +\left\langle\sin \left(\phi-\phi_{S}\right)\right\rangle_{\mathrm{UT}}^{\mathrm{h}} \sin \left(\phi-\phi_{S}\right) .
\end{aligned}
$$

This simultaneous extraction of both contributions was shown by detailed Monte Carlo simulations to avoid significant crosscontamination, even when they have very different magnitudes in the context of a limited detector acceptance. These initial data on the proton target provided evidence for both the Collins and Sivers signals. On the other hand, asymmetries measured by COMPASs using higher energy muons on a deuteron target are consistent with zero [115]. As illustrated in figures 17 and 21 , the non-zero results for the proton are now firmly established by recent more precise preliminary data [108], while the most recent results for the deuteron remain consistent with zero [109]. For the proton target, the Collins azimuthal amplitude for $\pi^{+}$is clearly positive and non-zero, while it is negative and at least as large for $\pi^{-}$. The latter finding was a surprise because of the contrast with the double-spin longitudinal asymmetries shown in figure 7 . In that case asymmetries for pions of both charges are positive, even though $\Delta u$ is positive and $\Delta d$ is negative, albeit smaller in magnitude. The reason there is that both charges are produced mostly from photoabsorption by $u$ quarks [63], but not as dominantly for $\pi^{-}$. Lattice calculations and models predict that transversity densities resemble the helicity densities, at least at large $x$ where valence quarks dominate, and where the $\pi^{+} / \pi^{-}$contrast is most apparent in figure 17 . Hence efforts to find an explanation focused on the Collins fragmentation function.

The explanation that emerged for the larger negative $\pi^{-}$ amplitudes is a substantial magnitude for the disfavoured Collins function describing, e.g., the fragmentation of $u$ quarks to $\pi^{-}$mesons, and with a sign opposite to that of the favoured function. Opposite signs could be expected in the light of the string model of fragmentation discussed in section 3.5. If a favoured pion forms as the string end created by the first break, a disfavoured pion from the next break will inherit transverse momentum from the first break in the opposite
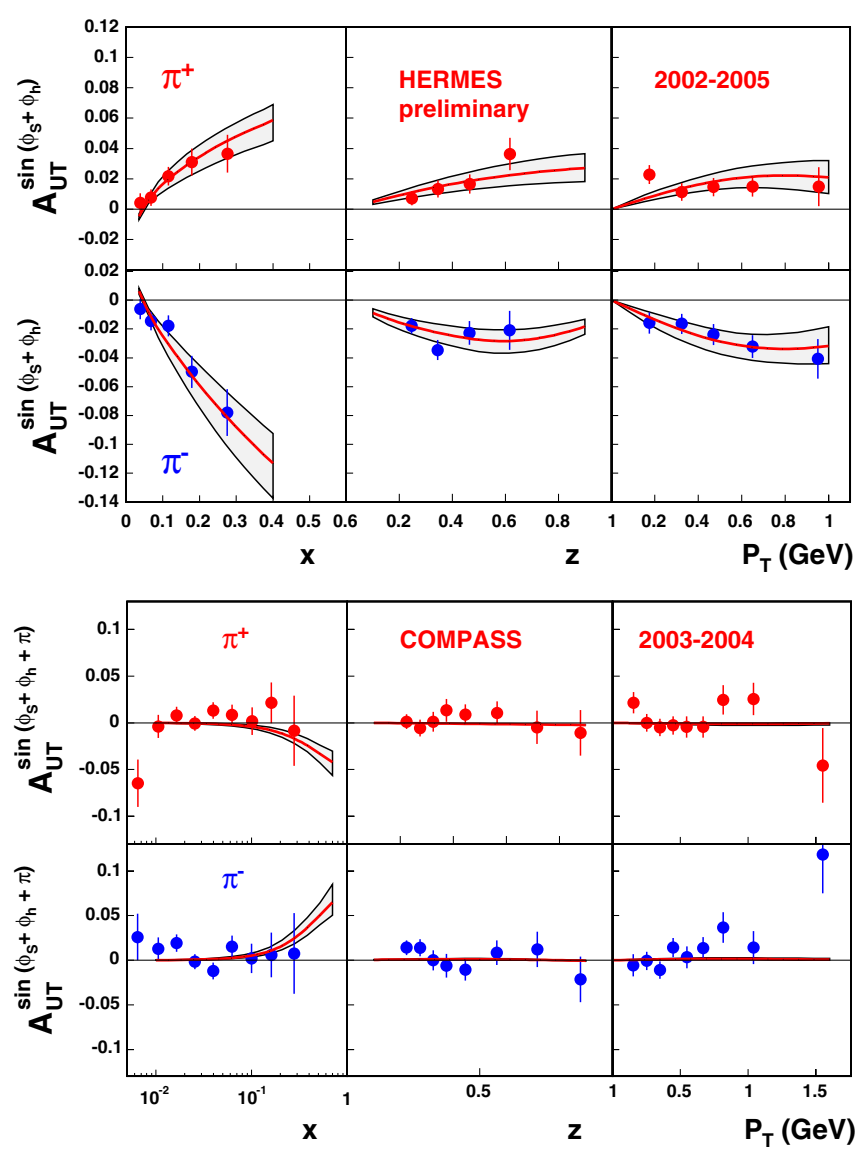

Figure 17. Collins sinusoidal amplitude of the lepton-beam asymmetry with respect to transverse polarization of a proton [108] (deuteron [109]) target, for the semi-inclusive production by positrons (muons) of identified charged mesons as labelled in the upper (lower) panel, as a function of $x, z$ or $p_{\mathrm{h}, \mathrm{T}}$ (the latter appearing as $P_{\mathrm{T}}$ in the figure). The sign of the amplitudes is according to the Trento conventions [110], although for the deuteron target the sign is reversed because of the extra value of $\pi$ added to the argument of the sin function. The error bars represent the statistical uncertainties, while for the proton target there is an additional common $8 \%$ scale uncertainty. The curves are fits to the data, as described in the text [111]. The error bands on these curves arise from the statistical uncertainties of the data, but the procedure chosen implies that they are several times larger than the

conventional definition given by, e.g. the Particle Data Group [71]. On the other hand, uncertainties related to model assumptions are not estimated. The figure is taken from [111].

direction from that acquired by the first pion. Such a $\boldsymbol{p}_{\pi, \mathrm{T}}$ anticorrelation between favoured and disfavoured pions is demonstrated by the JETSET simulation [116], which is based on a string fragmentation model. Hence any correlation between $\boldsymbol{p}_{\pi, \mathrm{T}}$ and another kinematic or spin observable should have the opposite sign for favoured and disfavoured pions.

This expectation for the Collins function has recently been confirmed by results for both favoured and disfavoured functions from a simultaneous fit of these DIS data together with information about the azimuthal structure of jets in $\mathrm{e}^{+} \mathrm{e}^{-}$ collisions from the BeLLE collaboration [112]. Two alternative jet asymmetries extracted from the BELLE data are shown in figure 18. The most recent such fit [111] is shown as the curves in figures 17 and 18. Such fits of data from these three 

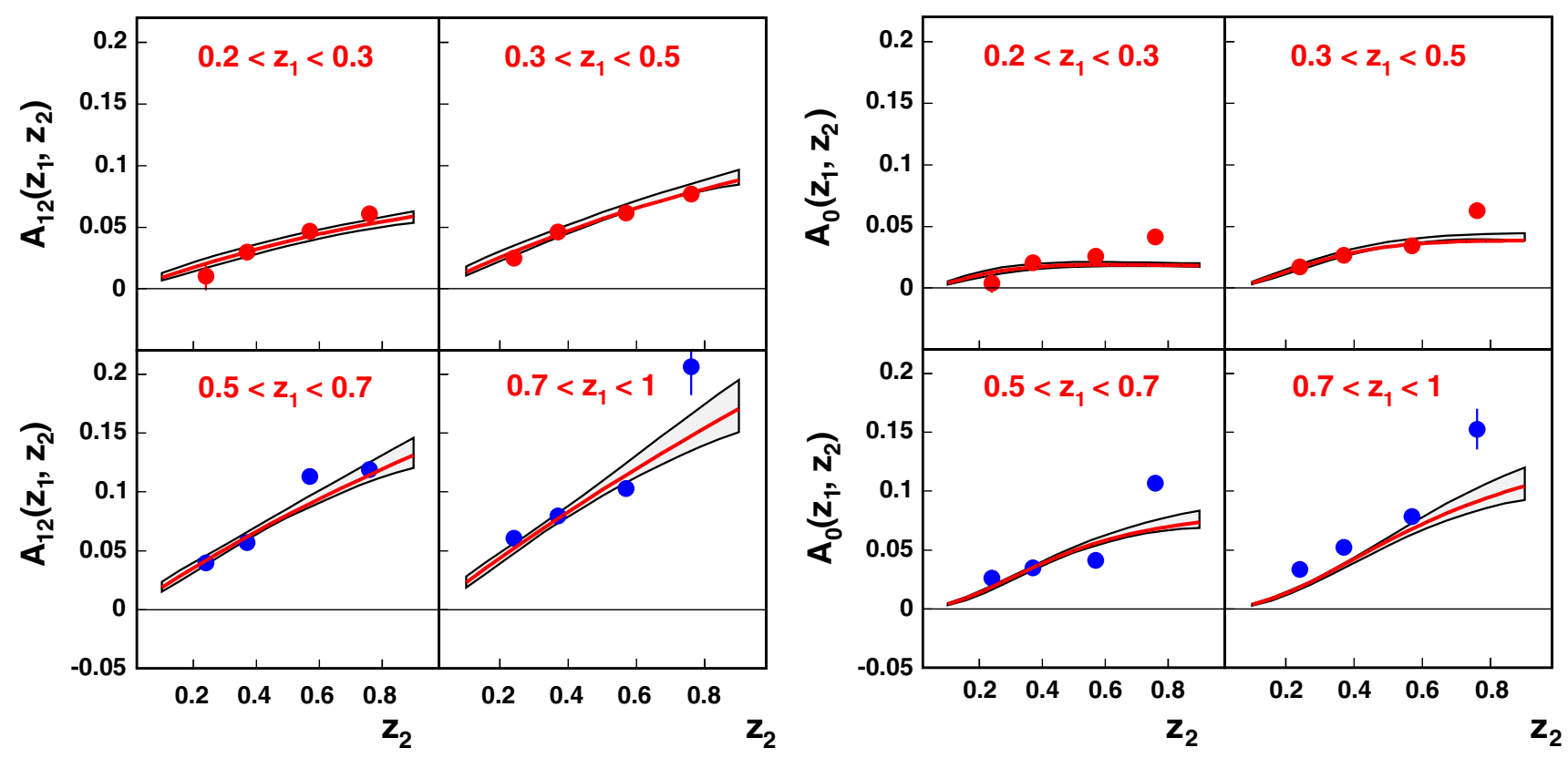

Figure 18. Two alternative jet asymmetries extracted from BelLE data [112] for $\mathrm{e}^{+} \mathrm{e}^{-} \rightarrow 2$ jets, compared with a fit [111] of parametrized Collins functions. The figure is taken from [111].
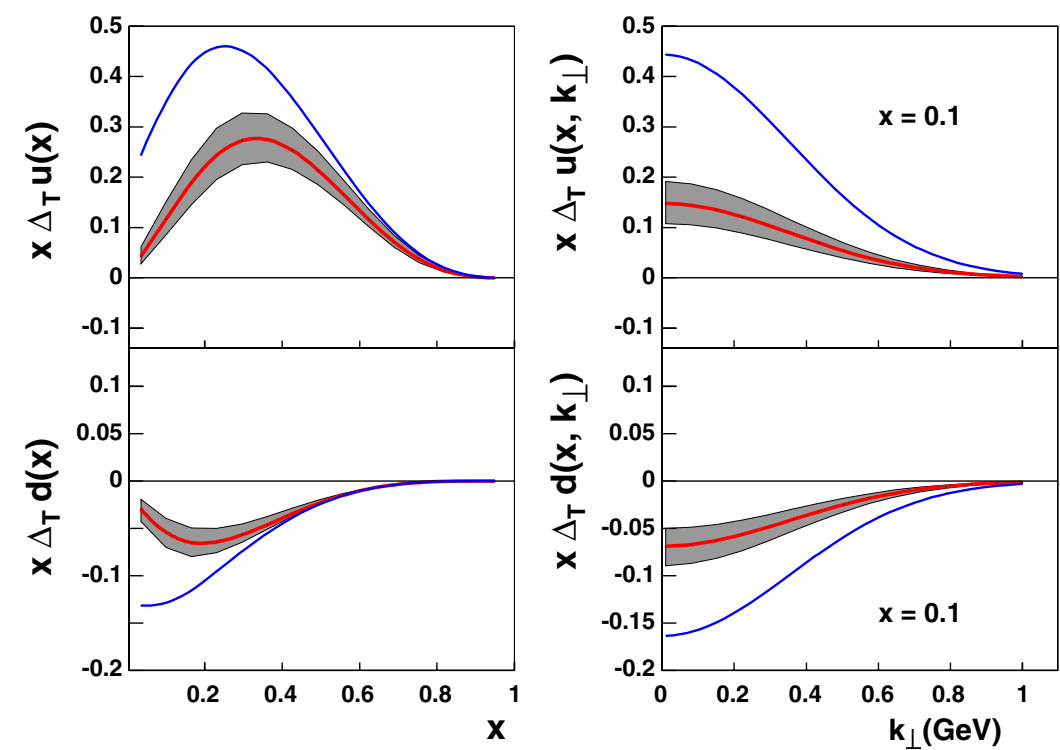

Figure 19. The (red) curves within the error bands represent values of the transversity distributions at $Q^{2}=2.4 \mathrm{GeV}^{2}$ for $u$ (upper panels) and $d$ (lower panels) quarks as a function of $x$ and $p_{\mathrm{T}}$ (labelled $k_{\perp}$ in the figure), from the fit [111] to the pion data shown in figure 17 together with $\mathrm{e}^{+} \mathrm{e}^{-}$data from Belle [112]. The $p_{\mathrm{T}}$ dependence in the right panels was chosen to be the same as that used for the unpolarized distributions, which was derived from fitting the azimuthal dependence of unpolarized SIDIS cross sections. The 'Torino notation' used in the figure for the transversity distributions is equivalent to the 'Amsterdam notation' used elsewhere in this paper. (This is not true for, e.g., TMDs [110].) The outermost (blue) curves represent the Soffer positivity bounds [85]. The error bands have the same meaning as in figure 17. The figure is taken from [111].

experiments produced the first experimental determination of the transversity distributions of $u$ and $d$ quarks, the most recent example of which is shown in figure 19. At this stage, the quark sea is neglected. As is the case for the helicity distributions, transversity is found to be positive for $u$ quarks and negative and smaller in magnitude for $d$ quarks. For both flavours, the magnitudes are of the order of half of the Soffer positivity bound [85]. Especially for $u$ quarks and for larger values of $x$ (bearing in mind that there are no data for $x>0.3$ ), the extracted density is smaller than the existing model predictions [117-119]. This fit also yields values for the Collins fragmentation function, shown in figure 20. They confirm that indeed the disfavoured Collins function is opposite in sign and larger in magnitude than the favoured function. The initially surprising features of the Collins asymmetry data now appear to be well understood. A further confirmation of 

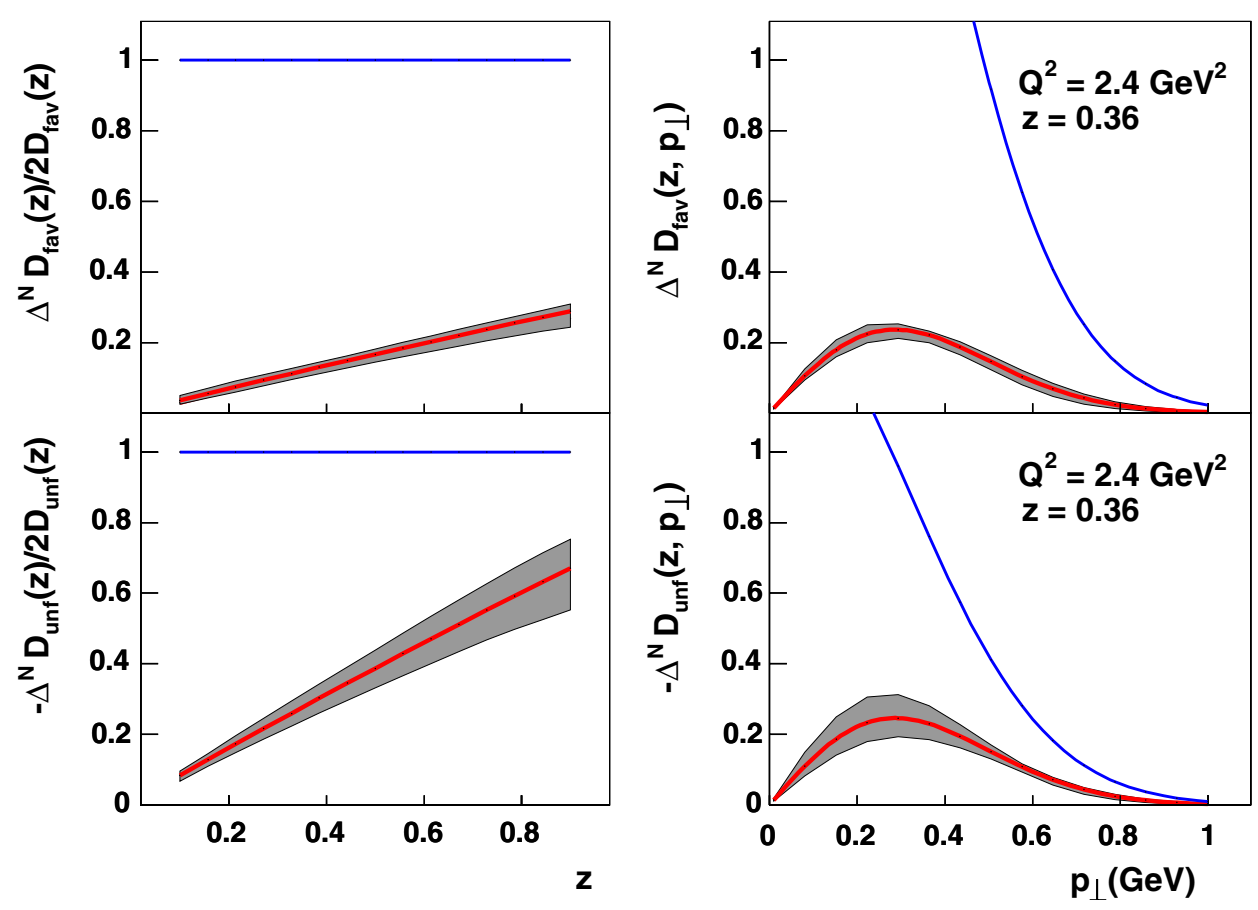

Figure 20. The (red) curves within the error bands represent values of the favoured and disfavoured Collins fragmentation functions at $Q^{2}=2.4 \mathrm{GeV}^{2}$ as functions of $z$ or $k_{\mathrm{T}}$ (the latter appearing in the figure as $p_{\perp}$ ), from the fit [111] to the pion data shown in figure 17 , together with $\mathrm{e}^{+} \mathrm{e}^{-}$data from BELLE [112]. In the left panels, the $k_{\mathrm{T}}$-integrated functions are shown as ratios to twice the corresponding unpolarized fragmentation functions, while in the right panels, the functions are evaluated at fixed values of $z$. The Collins function is represented in the Torino notation, which is related to the Amsterdam notation used elsewhere in this paper by $\Delta^{N} D_{\mathrm{h} / q^{\uparrow}}\left(z, p_{\mathrm{h}, \mathrm{T}}^{2}\right)=\left(2\left|p_{\mathrm{h}, \mathrm{T}}\right| / z M_{\mathrm{h}}\right) H_{1}^{\perp q}\left(z, p_{\mathrm{h}, \mathrm{T}}^{2}\right)[110]$. The uppermost (blue) curves represent the positivity limits. The error bands have the same meaning as in figure 17. The figure is taken from [111].

this recently appeared in the form of preliminary single-spin asymmetry data for unidentified hadrons from the proton by the Compass collaboration [120]. These data for the Collins asymmetry are consistent with predictions based on this fit of earlier data from HERMES on the proton and from COMPASS on the deuteron.

The Sivers azimuthal amplitudes for production of both pions and kaons from the proton shown in the upper panels of figure 21 are clearly positive and non-zero for the positive charges. This constitutes the first evidence from leptoproduction for a non-zero T-odd PDF. We note here that these Sivers amplitudes receive no contributions that are suppressed by $1 / Q$ (twist-3) [91]. Since the production of both $\pi^{+}$and $\mathrm{K}^{+}$is dominated by $u$ quarks, this positive value with the definition of azimuthal angles used here implies a negative value for the Sivers function $f_{1 \mathrm{~T}}^{\perp}$ of this flavour [110]. The amplitudes for the negatively charged mesons are consistent with zero. Their interpretation is complicated by competition between fragmentation of quarks of at least two flavours. Remarkably, for the proton target, the $\mathrm{K}^{+}$amplitudes are on average much larger than those for $\pi^{+}$, even though one might suppose that they are similarly dominated by photoabsorption by $u$ quarks. The amplitudes for the deuteron target measured by COMPASS are all consistent with zero (as are their preliminary Sivers amplitudes on the proton for unidentified hadrons [120]).

The curves on these figures from [113] represent an 11-parameter fit to data for pions and kaons from Hermes on the proton and from COMPASS on the deuteron, extracting representations of the Sivers functions for all six quark flavours. The shapes of all four sea flavours $\bar{u}, \bar{d}, s$ and $\bar{s}$ were constrained to be the same. The success in explaining the large $\mathrm{K}^{+}$ amplitudes for the proton depends on the use of a recent fit of unpolarized fragmentation functions [121], which differs from all previous such fits in that $D_{\bar{s}}^{\mathrm{K}^{+}}(z) \gg D_{u}^{\mathrm{K}^{+}}(z)$ over the whole $z$ range. This feature implies that $\mathrm{K}^{+}$production need not be dominated by $u$ quarks, allowing $\bar{s}$ quark distributions to be probed.

Values of the Sivers functions resulting from the fit are shown in figure 22. The Sivers function for $d$ quarks is found to be at least as large in magnitude as that for $u$ quarks. Their signs agree with expectations based on an attractive FSI between the struck quark and spectator diquark (see section 3.4). Such a relatively large magnitude of the Sivers function for $d$ quarks could be explained by a similar trend in the known relative contributions of $u$ and $d$ quarks to the anomalous magnetic moment of the proton, as explained in section 4.3. Hence the data appear to support a profound connection between static and partonic properties of the proton. Furthermore, there is evidence for a significant role of sea quarks. In considering the statistical significance of this finding, it is useful to bear in mind that the method of assigning the error bands results in values much larger than those that would correspond to the definition of, e.g., the Particle Data Group [71]. On the other hand, uncertainties related to model assumptions are not estimated.

We now look forward to measurements of single-spin asymmetries for the DY process, from which values of the 

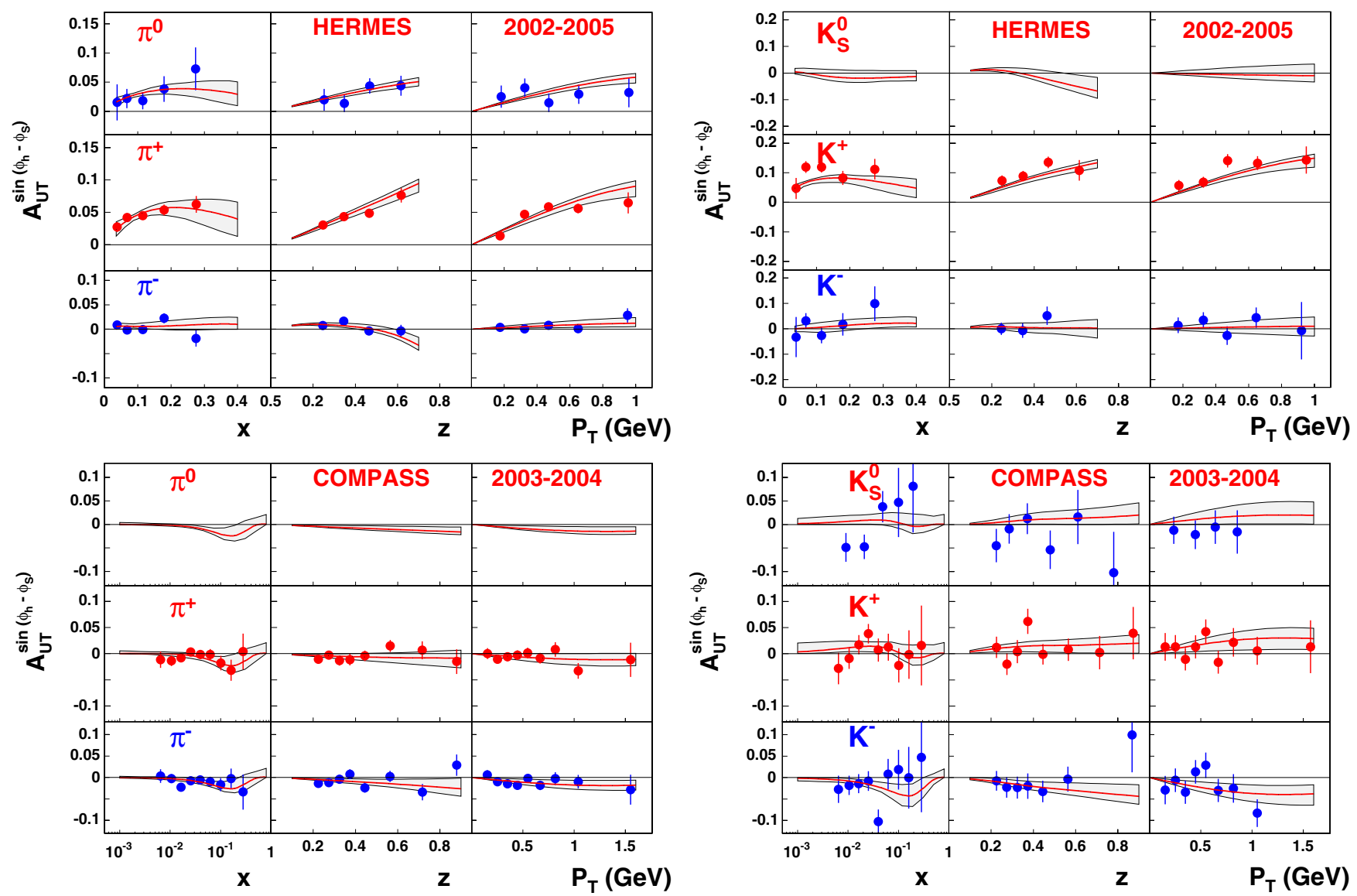

Figure 21. The Sivers sinusoidal amplitude of the asymmetry with respect to transverse polarization of a proton [108] (deuteron [109]) target, for the semi-inclusive production by positrons (muons) of identified charged mesons as labelled in the upper (lower) panels, as a function of $x, z$ or $p_{\mathrm{h}, \mathrm{T}}$ (the latter appearing as $P_{\mathrm{T}}$ in the figure). The sign of the amplitudes is according to the Trento conventions [110]. The error bars represent the statistical uncertainties, while for the proton target there is an additional common $8 \%$ scale uncertainty. The curves are fits to the data, as described in the text [113]. The error bands on these curves arise from the statistical uncertainties of the data, but the procedure chosen implies that they are several times larger than the conventional definition given by, e.g., the Particle Data Group [71]. On the other hand, uncertainties related to model assumptions are not estimated. The figure is taken from [113].

Sivers function can be extracted that are predicted on the basis of fundamental properties of QCD to be equal in magnitude but opposite in sign to those from DIS, as in (23). This will test our basic understanding of T-odd PDFs within the context of QCD. Thus continues the exciting story of a feature of proton structure that only a few years ago was (incorrectly) asserted to be forbidden by fundamental time reversal invariance [98].

\section{Towards a three-dimensional picture of the nucleon}

\subsection{Generalized parton distributions}

In section 1 it was explained that factorization can be applied to the theoretical interpretation of DIS cross sections. Using the optical theorem, the inclusive DIS cross section can be related to the imaginary part of the forward Compton amplitude, which can also be factorized into a 'hard part' describing the vertices of the active quark with the photons and the quark propagating between these two vertices, and a soft part describing the quark correlation function in the hadron (see figure 23(a)). A similar factorization of the scattering amplitude occurs in DVCS [122], $\gamma^{*} \mathrm{p} \longrightarrow \gamma \mathrm{p}$, where $\gamma^{*}$ is a space-like photon with virtuality $q^{2}=-Q^{2}<0$. As in DIS, the process is best interpreted in a frame in which the nucleon has infinite momentum opposite to that of the virtual photon. In the Bjorken limit, and for a momentum transfer $t$ to the proton much less than $Q^{2}$, the DVCS amplitude factorizes into a convolution of the Compton amplitude for scattering off a quark constituting the hard part, and a quark correlation function, constituting the soft part of the amplitude. After factorization has been applied to the DVCS amplitude, its matrix element has much in common with the matrix element describing the form factor (figure 23(b)). Indeed, the soft part entering the DVCS amplitude becomes identical to the soft part entering the form factor. However, the DVCS process provides more 'surgical' access. In the case of the DVCS amplitude, the hard part has a dependence on the quark momentum fraction appearing in the quark propagator, which is not present in the form factor. The additional kinematic degree of freedom provided by the hard part in the DVCS process provides access to the dependence of the soft part on the momentum fraction $x$ of the active quark. This motivates the concept of GPDs as a description of the soft part that enters the DVCS amplitude [123]. What is gained by introducing this concept 

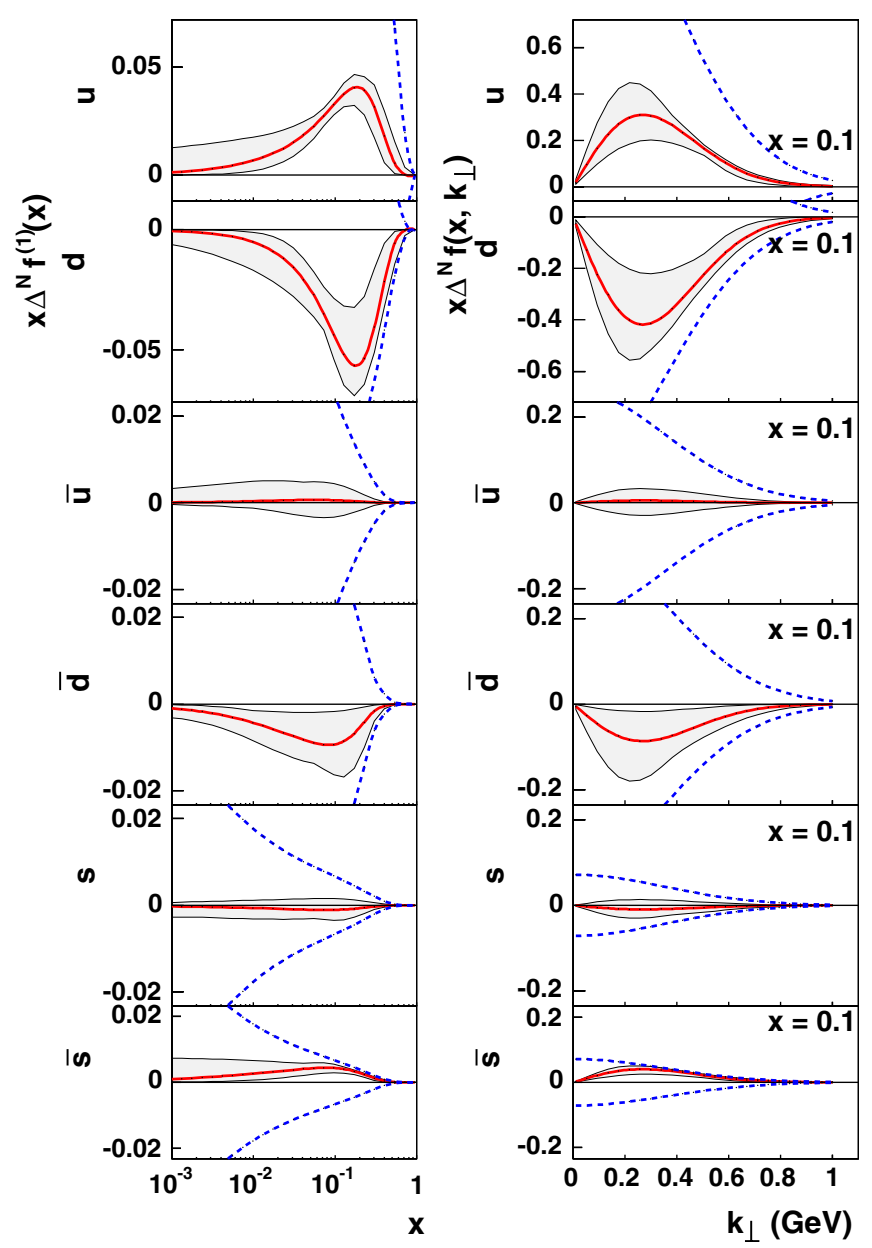

Figure 22. The left (right) panels show as continuous (red) curves within the error band values of the first $p_{\mathrm{T}}[x]$ moment of the Sivers function as functions of $x$ ( $p_{\mathrm{T}}$, appearing in the figure as $k_{\perp}$ ), from a fit [113] to the data shown in figure 21. The Sivers function is represented in the Torino notation, which in this case is opposite in sign and equal in magnitude to that in the Amsterdam notation used elsewhere in this paper. The error bands have the same meaning as in figure 21. The figure is taken from [113].

is that universality as introduced in section 1 applies with an extended scope, now also encompassing form factors and hard exclusive processes - the same GPDs that describe elastic form factors as well as the soft part of DVCS should also appear in other hard exclusive reactions. In each of these reactions, they appear convoluted with the appropriate coefficient function representing the hard part. In section $4.5 \mathrm{ff}$, it is discussed how and to what extent exclusive reactions can be used to constrain GPDs experimentally.

In elastic scattering, the photon can couple to any of the quark lines connecting the two 'soft blobs' in figure 23(b), leaving the nucleon intact. The form factor is the coherent superposition of such diagrams for all the quarks of all flavours in the nucleon. The experimental decomposition of the nucleon form factor into the contributions from various quark flavours has been partially accomplished through the comparison of proton and neutron form factors using isospin symmetry, and the interpretation of parity-violating electron scattering. Two flavour combinations $(2 u-d-s)$ and $2(d-u-s)$ are obtained from the proton and neutron electromagnetic form factors

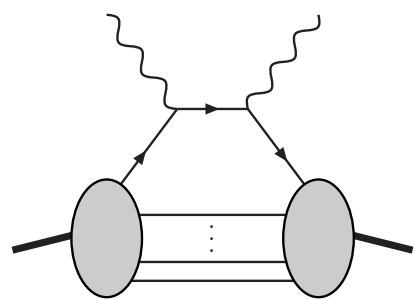

(a)

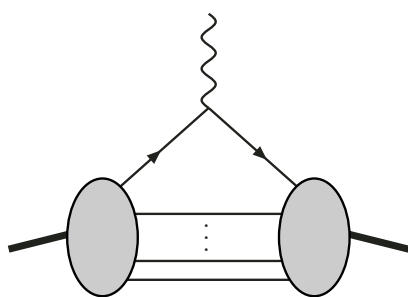

(b)
Figure 23. Comparison between (a) the Compton amplitude with two photon vertices and a quark propagating between them and $(b)$ a form factor with just one photon vertex.

using charge symmetry. A further combination is accessible in parity violating electron scattering, but the corresponding constraints are not very strong yet [124].

However, what is still needed for a more comprehensive understanding of the microscopic physics that underlies form factors is their decomposition with respect to the momentum fraction of the quark that absorbs the photon. GPDs embody this information. By definition, they provide a decomposition of form factors evaluated at a given value of the invariant momentum transfer $t=\left(p_{\text {fin }}-p_{\text {ini }}\right)^{2}$ on the target, with respect to the average momentum fraction $x=\frac{1}{2}\left(x_{\mathrm{ini}}+x_{\mathrm{fin}}\right)$ of the active quark of flavour $f$ :

$$
\begin{aligned}
& \int \mathrm{d} x H^{f}(x, \xi, t)=F_{1}^{f}(t) \quad \int \mathrm{d} x E^{f}(x, \xi, t)=F_{2}^{f}(t) \\
& \int \mathrm{d} x \widetilde{H}^{f}(x, \xi, t)=G_{\mathrm{A}}^{f}(t) \quad \int \mathrm{d} x \widetilde{E}^{f}(x, \xi, t)=G_{\mathrm{P}}^{f}(t),
\end{aligned}
$$

where $x_{\text {ini }}$ and $x_{\text {fin }}$ are the initial and final momentum fractions of the active quark with respect to the average momentum $\frac{1}{2}\left(p_{\text {ini }}+p_{\text {fin }}\right)$, and the dimensionless 'skewness' $\xi=\frac{1}{2}\left(x_{\mathrm{ini}}-\right.$ $\left.x_{\text {fin }}\right)$ represents their difference. Thus the linear combinations $x \pm \xi$ represent the momentum fraction carried by the quark before/after the hard interactions. The functions $F_{1}^{f}(t), F_{2}^{f}(t)$, $G_{\mathrm{A}}^{f}(t)$ and $G_{\mathrm{P}}^{f}(t)$ are the contributions to the Dirac, Pauli, axial and pseudoscalar form factors from quark flavour $f$, respectively, and the functions $H^{f}, E^{f}, \widetilde{H}^{f}$ and $\widetilde{E}^{f}$ are the quark GPDs providing their momentum decomposition. One can thus imagine the form factor being the result of adding (or integrating over) the contributions from all possible quark momentum fractions $x$ (here $x$ is not to be identified with the Bjorken variable $x_{\mathrm{B}}$ defined in section 1).

Just like the ordinary (forward) PDFs, GPDs are subject to higher order corrections in $\alpha_{s}$ and are thus also dependent on the scale $Q^{2}$ (and implicitly also on the factorization scheme used) at which they are probed. Nevertheless, as $F_{1}^{f}(t)$ and $F_{2}^{f}(t)$ are defined as matrix elements of a conserved current, they are $Q^{2}$ independent and hence the $Q^{2}$ dependence must disappear in the lowest moment of the corresponding GPDs (32).

Decomposing the form factors with respect to the quark momentum fraction $x$ involves the infinite-momentum direction. Hence it can make a difference whether the momentum transfer to the target is parallel, or perpendicular 


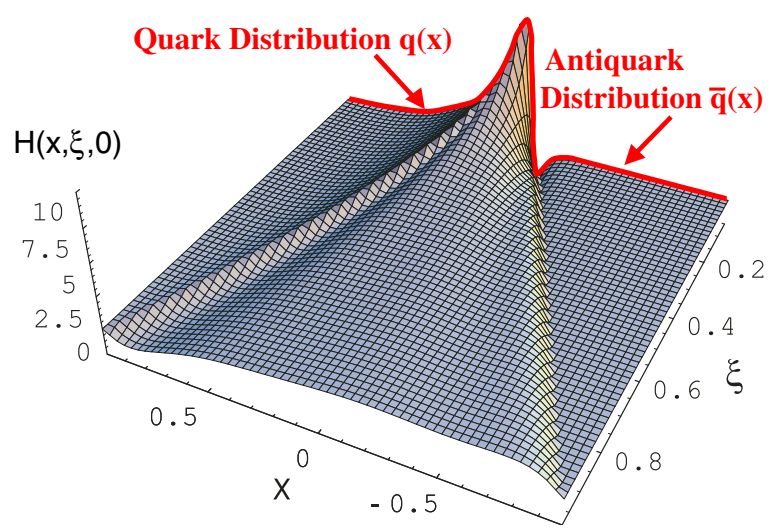

Figure 24. Model calculation for $H^{q}(x, \xi, 0)$ from [125]. The thick (red) line represents the forward limit $\xi=0$. Accessible to the DVCS process is the 'outer' or 'DGLAP' region $|x|>\xi$ that represents quarks for $x>\xi$ and antiquarks for $x<-\xi$. In the 'inner' or 'ERBL' region $|x|<\xi$, the quark GPD behaves like a meson distribution amplitude in deeply virtual meson production. Reprinted from [125], copyright (2001), with permission from Elsevier.

to this momentum. The GPDs must therefore depend on an additional variable, here called $\xi$. Its relationship to $t / M^{2}$ characterizes the direction of the momentum transfer relative to the infinite momentum direction. The case $\xi=0$ corresponds to the case where those two momenta are perpendicular to each other (which is not directly experimentally accessible in DVCS), while non-zero $\xi$ corresponds to the situation where the momentum transfer to the target has a component parallel to the virtual photon direction. Very little is known about the full $x$ versus $\xi$ dependence of GPDs, except that they might possibly be quite complex, as indicated, for example, by the model calculation shown in figure 24. In (32) and (33), the integration over $x$ removes all reference to the infinite momentum direction, with respect to which $x$ and $\xi$ are defined. Lorentz invariance thus implies that the $\xi$ dependence disappears in these integrals.

One constraint on quark GPDs comes from the fact that in the limit of vanishing $t$ and $\xi$, the nucleon helicity non-flip quark GPDs must reduce to the respective PDFs:

$$
\begin{array}{ll}
H^{f}(x, 0,0)=q_{f}(x), & \widetilde{H}^{f}(x, 0,0)=\Delta q_{f}(x) \\
\quad \text { for } x>0 & \\
H^{f}(x, 0,0)=-\bar{q}_{f}(-x), & \widetilde{H}^{f}(x, 0,0)=\Delta \bar{q}_{f}(-x) \\
\quad \text { for } x<0 . &
\end{array}
$$

GPDs became the focus of intense interest after the discovery of the 'Ji relation', connecting a second moment of GPDs with the total angular momentum of quarks with flavour $f$ or gluons, which is the respective contribution to the nucleon spin $\frac{1}{2}[19]$ :

$$
\frac{1}{2}=\sum_{f} J_{f}+J_{\mathrm{G}},
$$

where

$$
\begin{aligned}
& J_{f}=\frac{1}{2} \lim _{t \rightarrow 0} \int_{-1}^{1} \mathrm{~d} x x\left[H^{f}(x, \xi, t)+E^{f}(x, \xi, t)\right], \\
& J_{\mathrm{G}}=\frac{1}{2} \lim _{t \rightarrow 0} \int_{0}^{1} \mathrm{~d} x\left[H^{\mathrm{G}}(x, \xi, t)+E^{\mathrm{G}}(x, \xi, t)\right] .
\end{aligned}
$$

Note that no $\xi$ dependence remains in (37) and (38) after integration over $x$, although the two unpolarized quark GPDs $H^{f}$ and $E^{f}$ have to be evaluated at some common value of $\xi$. Unlike the lowest moments (32), these second moments and hence $J_{f, \mathrm{G}}$ depend on $Q^{2}$ (as well as order in $\alpha_{s}$ and factorization scheme), but this dependence is usually suppressed for simplicity.

Equation (37) is usually applied to the $\hat{z}$ component of the quark total angular momentum in a longitudinally polarized nucleon. However, in principle (37) can be applied for any polarization direction of the nucleon target. Nevertheless, (37) finds its most important application in the helicity sum rule through

$$
J_{f}=\frac{1}{2}\left(\Delta q_{f}+\Delta \bar{q}_{f}\right)+L_{f},
$$

since in that case at least the quark helicity contribution $\Delta q_{f}+\Delta \bar{q}_{f}$ in $J_{f}$ has a partonic interpretation. (An attempt to associate a partonic interpretation of (37) in the case of transversely polarized nucleons can be found in [126].)

The gauge invariant orbital angular momentum $L_{f}$ of quarks is defined through the expectation value of $\psi_{f}^{\dagger} \vec{r} \times(\vec{p}-g \vec{A}) \psi_{f}$. No process has been identified to measure $L_{f}$ directly, but it can be evaluated indirectly through (39) as

$$
L_{f}=J_{f}-\frac{1}{2}\left(\Delta q_{f}+\Delta \bar{q}_{f}\right)
$$

The issues arising in a decomposition of the nucleon spin are further discussed in section 5 .

\subsection{General properties of GPDs}

GPDs must satisfy several relations arising from fundamental symmetries or conservation laws:

- Invariance under time reversal (for both quark and gluon GPDs):

$$
F(x,-\xi, t)=F(x, \xi, t),
$$

- Polynomiality property of GPDs [127] as a consequence of Lorentz invariance:

$$
\begin{gathered}
\int_{-1}^{1} \mathrm{~d} x x^{n-1} H^{f}(x, \xi, t)=\sum_{\substack{k=0 \\
\text { even }}}^{n-1}(2 \xi)^{k} A_{n, k}^{f}(t) \\
+\bmod (n-1,2)(2 \xi)^{n} C_{n}^{f}(t), \\
\int_{-1}^{1} \mathrm{~d} x x^{n-1} E^{f}(x, \xi, t)=\sum_{\substack{k=0 \\
\text { even }}}^{n-1}(2 \xi)^{k} B_{n, k}^{f}(t) \\
-\bmod (n-1,2)(2 \xi)^{n} C_{n}^{f}(t) .
\end{gathered}
$$

Polynomiality means that the entire $\xi$ dependence of the $n$th $x$-moment $(n>0)$ of a GPD is given by a polynomial in $\xi$ of order at most $n$. Polynomiality is satisfied, e.g., by the representation of GPDs in terms of double distributions (DDs) $F_{\mathrm{DD}}^{f}(\beta, \alpha, t)$ [128-130], which can be considered as spectral functions of non-forward matrix elements. In 
terms of a DD, a generic quark GPD $F$ can always be written as

$$
\begin{aligned}
& F^{f}(x, \xi, t) \\
& \quad=\int_{-1}^{1} \mathrm{~d} \beta \int_{-1+|\beta|}^{1-|\beta|} \mathrm{d} \alpha \delta(x-\beta-\xi \alpha) F_{\mathrm{DD}}^{f}(\beta, \alpha, t) \\
& \quad+\theta(\xi-|x|) D^{f}\left(\frac{x}{\xi}, t\right) .
\end{aligned}
$$

The step function $\theta(\zeta)$ is zero for $\zeta<0$ and unity for $\zeta>0$. Thus the so-called ' $D$-term' $D^{f}$ [127] contributes only in the 'ERBL' region $-\xi<x<\xi$, where quark GPDs have the character of distribution amplitudes for the creation of a quark/antiquark pair. It does not contribute in the complementary so-called 'DGLAP' region $|x|>\xi$, where quark GPDs describe the emission and reabsorption of an (anti-)quark in the infinitemomentum frame, thereby having properties analogous to the familiar (anti-)quark distribution functions. The $D$-term provides a convenient means of representing this profound difference in GPD properties between the two regions, which would otherwise require singularities in the DD having a severity beyond representation by delta functions or their derivatives. Another perspective is that the maximum power of $\xi$ provided by the DD itself is one less than that allowed by Lorentz invariance. Expressions analogous to (44) hold for the polarized quark GPDs $\widetilde{H}$ and $\widetilde{E}$, for which the $D$-term vanishes. The mapping between GPDs and DDs is not one to one; each GPD can be represented by any of an infinite family of DDs. The quantity $C_{n}^{f}(t)$ in (42), (43) is the $n$th $x$-moment of $D^{f}(x / \xi, t)$, which has the same magnitude for the quark GPDs $H^{f}$ and $E^{f}$ but enters with the opposite sign. Hence the second moment of the sum of the unpolarized quark GPDs $H^{f}(x, \xi, t)+E^{f}(x, \xi, t)$ ('Ji integral' (37)) is independent of $\xi$.

Several sum rules apply at $\xi=0$ and $t=0$ [131]. Suppressing the kinematic arguments:

- Quark and gluon GPDs are related by the conservation of momentum,

$$
1=\int_{-1}^{1} \mathrm{~d} x x \sum_{f} H^{f}+\int_{0}^{1} \mathrm{~d} x H^{\mathrm{G}},
$$

and of the proton's angular momentum,

$$
\frac{1}{2}=\frac{1}{2} \int_{-1}^{1} \mathrm{~d} x x \sum_{f}\left(H^{f}+E^{f}\right)+\frac{1}{2} \int_{0}^{1} \mathrm{~d} x\left(H^{\mathrm{G}}+E^{\mathrm{G}}\right) .
$$

Combining them leads to

$$
0=\int_{-1}^{1} \mathrm{~d} x x \sum_{f} E^{f}+\int_{0}^{1} \mathrm{~d} x E^{\mathrm{G}} .
$$

- For each quark flavour, the GPD $E^{f}$ is constrained by the contribution $\kappa_{f}$ of flavour $f$ to the proton anomalous magnetic moment $\kappa$ :

$$
\int_{-1}^{1} \mathrm{~d} x E^{f}=F_{2}^{f}(t=0)=\kappa_{f} .
$$

As quark GPDs are not generally even or odd in $x$, it is often useful [131] to consider the GPD combinations

$H^{f( \pm)}(x, \xi, t) \equiv H^{f}(x, \xi, t) \mp H^{f}(-x, \xi, t)$,

$\widetilde{H}^{f( \pm)}(x, \xi, t) \equiv \widetilde{H}^{f}(x, \xi, t) \pm \widetilde{H}^{f}(-x, \xi, t)$,

and their analogues for $E^{f}$ and $\widetilde{E}^{f}$. Here the upper (lower) sign corresponds to the exchange of a $C=+1(C=-1)$ object in the $t$ channel, and denotes the 'singlet' ('non-singlet' [or valence]) combination. These names are taken from the corresponding forward limits given by

$$
\begin{gathered}
H^{f( \pm)}(x, 0,0)=q_{f}(x) \pm \bar{q}_{f}(x), \\
\widetilde{H}^{f( \pm)}(x, 0,0)=\Delta q_{f}(x) \pm \Delta \bar{q}_{f}(x),
\end{gathered}
$$

for $x>0$. While the singlet (C-even) combination of quark GPDs is probed in DVCS (see (68)) or exclusive vector meson production, the non-singlet (C-odd) one is selected in pseudoscalar meson production.

Gluons are their own antiparticles so that gluon GPDs are either even in $x\left(H^{\mathrm{G}}, E^{\mathrm{G}}\right)$ or odd $\left(\widetilde{H}^{\mathrm{G}}, \widetilde{E}^{\mathrm{G}}\right)$. Their forward limits involve an additional factor of $x$, so that (for $x>0$ ):

$H^{\mathrm{G}}(x, 0,0)=x \mathrm{G}(x), \quad \widetilde{H}^{\mathrm{G}}(x, 0,0)=x \Delta G(x)$.

\subsection{Impact-parameter-dependent parton distributions}

A particularly simple physical interpretation for GPDs can be developed in the limiting case $\xi=0$, where the quark momentum fraction is the same in the initial and final states. So far we have introduced GPDs as momentum-dissected form factors. Hence a GPD evaluated at a certain value of $x$ can be considered to be the form factor for only those quarks in the nucleon that carry momentum fraction $x$. In analogy with non-relativistic form factors, whose Fourier transform provides information on the charge distribution in position space, one would thus expect that the Fourier transform of the $t$ dependence of GPDs for fixed $x$ provides information on the position space distribution of quarks carrying a certain momentum fraction $x$. Indeed, it can be shown that the distribution of partons in impact parameter space can be obtained from GPDs via [20]

$$
q_{f}\left(x, \boldsymbol{b}_{\perp}\right)=\int \frac{\mathrm{d}^{2} \boldsymbol{\Delta}_{\perp}}{(2 \pi)^{2}} \mathrm{e}^{-\mathrm{i} \boldsymbol{\Delta}_{\perp} \cdot \boldsymbol{b}_{\perp}} H^{f}\left(x, 0,-\boldsymbol{\Delta}_{\perp}^{2}\right),
$$

where $-\Delta_{\perp}^{2}=t$ for $\xi=0$. Analogously, the parton helicity distribution in a longitudinally polarized target is obtained by replacing $q_{f} \rightarrow \Delta q_{f}$ and $H^{f} \rightarrow \widetilde{H}^{f}$. Note that the Heisenberg uncertainty principle forbids a simultaneous exact measurement of $x$ and the longitudinal (i.e. along the direction of the virtual photon) position of the quarks [22]. Therefore, only the impact parameter $\boldsymbol{b}_{\perp}$, i.e. the position transverse to the nucleon's momentum can be determined simultaneously with $x$. For the determination of the $\boldsymbol{b}_{\perp}$ distribution, the momentum transfer should be purely perpendicular, which implies the restriction to $\xi=0$. The reference point for the impact parameter $\boldsymbol{b}_{\perp}$ is the transverse centre of longitudinal momentum $\boldsymbol{R}_{\perp}$, which plays a role similar to the centre of mass in a non-relativistic system. In analogy to that case, it is obtained as an average of the positions $\boldsymbol{r}_{\perp, i}$ of all the partons, 
except that the weight factor is not the mass fraction, but the momentum fraction $x_{i}$ carried by each parton [132]:

$$
\boldsymbol{R}_{\perp}=\sum_{i=q, g} x_{i} \boldsymbol{r}_{\perp, i}
$$

For $x \rightarrow 1$, the active quark dominates the sum in (55) and $q\left(x, b_{\perp}\right)$ must necessarily become narrow in this limit (see figure 25).

In analogy to the inclusive PDFs that are measured in DIS, given that we have restricted ourselves to $\xi=0$, these impact-parameter-dependent parton distributions (IPD-PDFs or simply IPDs) have a probabilistic interpretation and hence they satisfy a variety of inequalities, such as [133,134]

$$
\begin{aligned}
& q_{f}\left(x, \boldsymbol{b}_{\perp}\right) \geqslant\left|\Delta q_{f}\left(x, \boldsymbol{b}_{\perp}\right)\right| \geqslant 0 \quad \text { for } x>0, \\
& -q_{f}\left(x, \boldsymbol{b}_{\perp}\right) \geqslant\left|\Delta q_{f}\left(x, \boldsymbol{b}_{\perp}\right)\right| \geqslant 0 \quad \text { for } x<0 .
\end{aligned}
$$

Remarkably, (54) is not limited to the non-relativistic domain of $|t|<m^{2}$, in contrast to the interpretation of form factors as three-dimensional Fourier transforms of charge distributions. Its only limitation is $|t| \ll Q^{2}$ as $1 / Q$ represents the spatial resolution of the virtual photon. Since the electromagnetic form factors are obtained as integrals of GPDs (see (32) and (33)), this feature of (54) implies that in the infinite-momentum frame, the identification of the two-dimensional Fourier transform of form factors with the charge distribution in the transverse plane is also not limited to $|t|<m^{2}$.

For a longitudinally polarized or unpolarized target, the distribution of quarks in the transverse plane is axially symmetric. However, when the target is transversely polarized, the polarization direction of the quark in the transverse plane breaks this axial symmetry. For example, for a target polarized in the $\hat{\boldsymbol{x}}$-direction, the quark distribution $q_{f}\left(x, \boldsymbol{b}_{\perp}\right)$ is deformed along the $\hat{\boldsymbol{y}}$-direction and the sign and magnitude of this deformation are embodied in the GPD $E^{f}\left(x, 0,-\Delta_{\perp}^{2}\right)[135]$ :

$$
\begin{gathered}
q_{f}\left(x, \boldsymbol{b}_{\perp}\right)=\int \frac{\mathrm{d}^{2} \boldsymbol{\Delta}_{\perp}}{(2 \pi)^{2}} \mathrm{e}^{-\mathrm{i} \boldsymbol{\Delta}_{\perp} \cdot \boldsymbol{b}_{\perp}} H^{f}\left(x, 0,-\boldsymbol{\Delta}_{\perp}^{2}\right) \\
+\frac{1}{2 M} \frac{\partial}{\partial b_{y}} \int \frac{\mathrm{d}^{2} \boldsymbol{\Delta}_{\perp}}{(2 \pi)^{2}} \mathrm{e}^{-\mathrm{i} \boldsymbol{\Delta}_{\perp} \cdot \boldsymbol{b}_{\perp}} E^{f}\left(x, 0,-\boldsymbol{\Delta}_{\perp}^{2}\right) .
\end{gathered}
$$

Such deformations can be interpreted semi-classically as an enhanced probability of photoabsorption occurring on the left or right of the nucleon as seen by the incident photon, when the nucleon spin is pointing e.g. up. As was explained in section 3.6, this suggests an intimate relationship between these deformations, parton orbital angular momentum and the Sivers effect.

Experimentally, only the $x$-integral of the GPD $E^{f}\left(x, 0,-\Delta_{\perp}^{2}\right)$, i.e. the form factor $F_{2}\left(-\Delta_{\perp}^{2}\right)$, is known (see (32)). Any additional constraints come only from lattice calculations, which will be discussed in the following section, as well as model studies. As a result, the details of the above deformation are not very well known. In the considered case of a transversely polarized target, this deformation implies the existence of a transverse flavour dipole moment. The integrated deformation can be related model-independently to

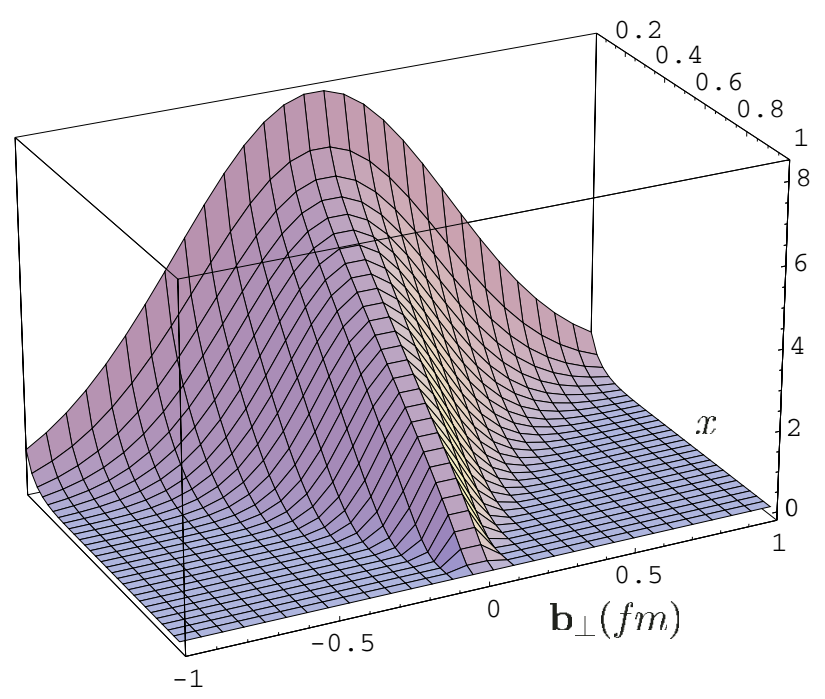

Figure 25. Qualitative shape of $q\left(x, \boldsymbol{b}_{\perp}\right)$.

the anomalous magnetic moment of the nucleon. For example, when the target is polarized in the $\hat{\boldsymbol{x}}$-(transverse) direction, the average deformation $\mathcal{D}_{f}$ of the distribution for quarks of flavour $f$ in the $\hat{\boldsymbol{y}}$ direction reads [135]

$$
\mathcal{D}_{f} \equiv \int_{-1}^{1} \mathrm{~d} x \int \mathrm{d}^{2} \boldsymbol{b}_{\perp} q_{f}\left(x, \boldsymbol{b}_{\perp}\right) b_{y}=\frac{\kappa_{f / \mathrm{p}}}{2 M} .
$$

The $\kappa_{f / p}$ are the contributions from quark flavour $f$ to the anomalous magnetic moment of the proton with the quark charge factored out: $\kappa_{\mathrm{p}}=\frac{2}{3} \kappa_{u / \mathrm{p}}-\frac{1}{3} \kappa_{d / \mathrm{p}}+\cdots \approx 1.79$. A flavour decomposition of the anomalous magnetic moment, using $\kappa_{n} \approx-1.91$, charge symmetry and neglecting the small contribution from $s$ quarks yields $\kappa_{u / \mathrm{p}} \approx 1.67$ and $\kappa_{d / \mathrm{p}} \approx-2.03$, which translates into average deformations of $\mathcal{D}_{u} \approx+0.16 \mathrm{fm}$ and $\mathcal{D}_{d} \approx-0.20 \mathrm{fm}$.

Such a significant transverse deformation of quark distributions should have observable consequences, e.g., in SIDIS on a transversely polarized target. Armed with the above quantitative information, the semi-classical arguments of section 3.6 about the transverse deformation of PDFs as a cause of single-spin asymmetries can be made more specific. When a virtual photon collides with a proton that has transverse spin down, it 'sees' more $u$ quarks on the right side of the proton and more $d$ quarks on the left side, with the signs being determined by the signs of $\kappa_{q / \mathrm{p}}$ (see figure 15 ). Since the FSI acting on the escaping quark is on average expected to be attractive, more ejected $u$ quarks are expected to be deflected to the left than to the right. For $d$ quarks, the opposite is predicted. The predicted negative sign of the Sivers function (22) for $u$ quarks $f_{1 \mathrm{~T}}^{\perp u \mathrm{p}}$ [100] has been confirmed by a recent analysis of SIDIS data for pion production on the proton $[108,114,115]$. Here the experimental result for $f_{1 \mathrm{~T}}^{\perp d / \mathrm{p}}$ is less clear as its contribution to pion production is strongly suppressed. The asymmetry on the deuteron is consistent with zero $[136,137]$. A small Sivers asymmetry could arise from cancellation between the contributions from the proton and neutron. Using charge symmetry this implies $f_{1 \mathrm{~T}}^{\perp u / \mathrm{n}} \approx-f_{1 \mathrm{~T}}^{\perp d / \mathrm{p}}$, which is consistent with the prediction based 
on the transverse deformation of IPDs. However, it is also possible that at very small $x$, both $f_{1 \mathrm{~T}}^{\perp u / \mathrm{p}}$ and $f_{1 \mathrm{~T}}^{\perp d / \mathrm{p}}$ are small.

\subsection{QCD simulations on a space-time lattice}

Ideally, in order to describe properties and interactions of hadrons theoretically, hadronic matrix elements should be evaluated in terms of QCD wave functions. However, this is not possible as long as wave functions in QCD cannot be calculated from first principles. QCD calculations on a Euclidean lattice (also known as lattice QCD or lattice gauge theory) are currently the most promising tool to overcome this problem. Presently there are a large number of workers active in this field - resulting in many types and flavours of lattice QCD. The proceedings of the yearly lattice symposia (currently published in $P O S$ ) provide a good overview of the state of the field; specifically concerning hadron structure, the two most recent summaries were reported in [138, 139].

The inevitable truncations for numerical computations in QCD are less problematic on a space-time lattice, where space rather than momentum is discretized. After discretizing spacetime on a four-dimensional grid, approximate solutions for hadron masses and matrix elements are obtained numerically using Monte Carlo techniques, because the latter are capable of dealing with the truly astronomical numbers of degrees of freedom (contemporary lattices typically have about $32^{3} \times 64$ discretization points; including spin and colour degrees of freedom, this implies a total of more than $10^{7}$ degrees of freedom for the entire lattice) that are necessary for an adequate approximation to the continuum. These Monte Carlo techniques are used to evaluate a so-called 'path integral', which sidesteps a direct computation of wave functions. For example, a pion form factor can be computed without having to know what a pion is, only its quantum numbers [140-143]. The evaluation of these path integrals would be impractical working with a real time variable, as amplitudes in quantum mechanics oscillate as a function of time and would thus lead to poor statistical precision. These oscillations are avoided by introducing an imaginary time $t \longrightarrow-\mathrm{i} \tau$, which renders the time (sic) evolution operator real and positive: $\exp (-\mathrm{i} H t) \longrightarrow$ $\exp (-H \tau)$, and thus suitable for Monte Carlo sampling. The variable $\tau$ is often referred to as 'Euclidean time', as the Minkowskian metric becomes Euclidean when expressed in terms of $(\tau, \vec{r})$. This last step requires an analytical continuation to translate the results into a Minkowskian spacetime.

It is impossible to apply lattice QCD to directly calculate DIS cross sections, since this would require a summation over all possible final states. Instead one can use the optical theorem and evaluate the imaginary part of the forward Compton amplitude. This requires the calculation of a correlation function $\left\langle P\left|\psi_{f}^{\dagger}(0) \gamma^{+} \psi_{f}\left(z^{-}\right)\right| P\right\rangle$ between nucleon states, where $\psi_{f}$ is the quark field operator. Here the lightcone variables are defined as $z^{ \pm}=\frac{1}{\sqrt{2}}\left(z^{0} \pm z^{3}\right)$, where the presence of $z^{0}$ makes the correlation function non-local in time. Such a space-time structure arises since the Compton amplitude involves two vertices in figure 23(a) coupling the nucleon to the virtual photon, separated in a light-like direction, as the struck quark moves with nearly the speed of light. This causes additional difficulties in the translation from imaginary time to real time. This problem restricts lattice gauge theory calculations of PDFs to only their Mellin moments. Such moments are accessible because they can be represented by matrix elements of local (i.e. containing products of field operators and their derivatives evaluated at the same spacetime point) operators for which this analytical continuation is trivial. For PDFs, which can apparently be parametrized by relatively simple functions, only a few moments may be sufficient to constrain these functions, except very close to the endpoints $x \longrightarrow 0,1$. In higher moments the region $x \longrightarrow 0$ is suppressed and they thus only indirectly help constrain this region. Even though higher moments emphasize the $x \longrightarrow 1$ region, PDFs are very small for very large $x$, and only very high moments yield useful constraints on the $x \longrightarrow 1$ behaviour of PDFs.

Parametrizing GPDs is significantly more difficult as there are two longitudinal variables instead of one, and the $t$ dependence adds yet another variable. Furthermore, it is not yet known if GPDs can be described by similarly simple functions as PDFs and hence it is not obvious how many moments will be needed to generate a reliable parametrization for GPDs. Indeed, both phenomenological model calculations (see figure 24) as well as perturbative QCD evolution [144] suggest a 'kink', i.e. a discontinuous first derivative, for GPDs at $x=$ $\pm \xi$, whose adequate parametrization may therefore demand a larger number of moments than was the case for PDFs.

Remarkable progress in lattice calculations has been made over the last three decades. One source of systematic uncertainties is the coarseness (spacing) of the lattice. Improved algorithms now permit improved precision at the same lattice spacing [145-149]. Another source of uncertainties arises from the finite size of the lattice (physical volume). Ideally, it should be large enough to contain the hadron state completely. Both because improved algorithms allow larger lattice spacings at the same precision and because increased computing power allows more discretization points, large lattices with several femtometres diameter are now possible. In the past, the omission of sea quarks in 'quenched calculations' gave rise to additional uncertainties. Steady increases in computational resources and improved algorithms make it now possible to also include sea quarks ('full' lattice QCD) and unquenched calculations are now routine. Nevertheless, calculating observables that require inserting operators into disconnected quark loops is at present still prohibitively expensive in most cases; some exceptions which are tractable have been studied, e.g., in [150]. Usually, contributions from disconnected diagrams are omitted, resulting in an uncontrolled approximation that affects most flavour singlet observables. Lattice calculations of flavour non-singlet observables are not affected by this omission and hence are much more reliable. For the Dirac form factor, the contribution from disconnected quark loops has been estimated to be small [151-154], consistent with the small influence from strange quarks on the charge radius of the nucleon $[155,156]$. For most other observables, it is not clear whether the disconnected quark loop contribution is small, but 
steady advances in computer technology will allow including those contributions in the near future.

The masses of light quarks $(f=u, d)$ are of the order of a few megaelectronvolts. Calculations are presently restricted to much higher values, corresponding to pion masses of $m_{\pi} \approx$ $250 \mathrm{MeV}$ or even higher ${ }^{10}$. This limitation is due not only to the increase in statistical fluctuations in the Monte Carlo algorithms for small quark masses but also to the increase in the required overall lattice volume: a proper description of the virtual pion fluctuations around a hadron demands lattices that are several pion Compton wavelengths in diameter. As a result of the restriction to performing calculations in an 'unphysical universe' in which the light quarks are too heavy, another significant source of uncertainty arises from the extrapolation to small quark masses and the difficulties involved in restoring the chiral symmetry (17) that was broken by an expedient necessary in discretizing the Dirac equation. Recently, new methods for approximating fermion fields on a lattice have been applied which provide significantly better approximations of chiral symmetry [157-159]. The latter advances now allow more meaningful calculations at smaller quark masses, where chiral symmetry becomes increasingly important. Fortunately, it is not necessary to perform lattice calculations at quark masses for $u$ and $d$ quarks of a few megaelectronvolts. Chiral perturbation theory $(\chi \mathrm{PT})$ is an effective field theory for QCD at small quark masses and momentum transfers. It can be used to extrapolate results from larger quark masses down to these values. However, a reliable extrapolation first requires lattice simulations for quark masses where $\chi$ PT can be trusted, a matter of controversy.

Lattice QCD calculations have now been used to evaluate a large variety of observables, and due to lack of space we present only a few selected cases. Figure 26 shows lattice results for the nucleon's axial charge $g_{\mathrm{A}}$ [160], which is related to the spin content of the nucleon by Bjorken's sum rule (8). These results illustrate not only the wide range of quark masses that is now accessible, but also the accuracy that can be achieved when extrapolating results to the physical value $m_{\pi}=139 \mathrm{MeV}$. For the tensor charge one finds after extrapolation to the chiral limit $\delta u-\delta \bar{u}=0.857 \pm 0.013$ and $\delta d-\delta \bar{d}=0.212 \pm 0.005$ [164].

Using lattice QCD calculations to determine the second moment of GPDs entering the Ji relation (37) yields $J_{u}=$ $0.214 \pm 0.027$ and $J_{d}=-0.001 \pm 0.027[165,166]$. Combining these results with lattice calculations for $\Delta q_{f}+\Delta \bar{q}_{f}$ one thus finds

$L_{u}^{\text {latt. }}=J_{u}^{\text {latt. }}-\frac{1}{2}\left(\Delta u^{\text {latt. }}+\Delta \bar{u}^{\text {latt. }}\right)=-0.195 \pm 0.044$,

$L_{d}^{\text {latt. }}=J_{d}^{\text {latt. }}-\frac{1}{2}\left(\Delta d^{\text {latt. }}+\Delta \bar{d}^{\text {latt. }}\right)=0.200 \pm 0.044$

at the physical pion mass where the uncertainties are only statistical since some of the systematic uncertainties are difficult to quantify. In these calculations, disconnected quark loops have been omitted in the calculation of the matrix elements. The perhaps surprising result that the net orbital angular momentum carried by the quarks is close to zero may thus be subject to large corrections. However, the isovector

${ }^{10}$ Since the quark mass itself is not an observable, results are usually presented as a function of the pion mass, which strongly depends on the masses of the quarks.

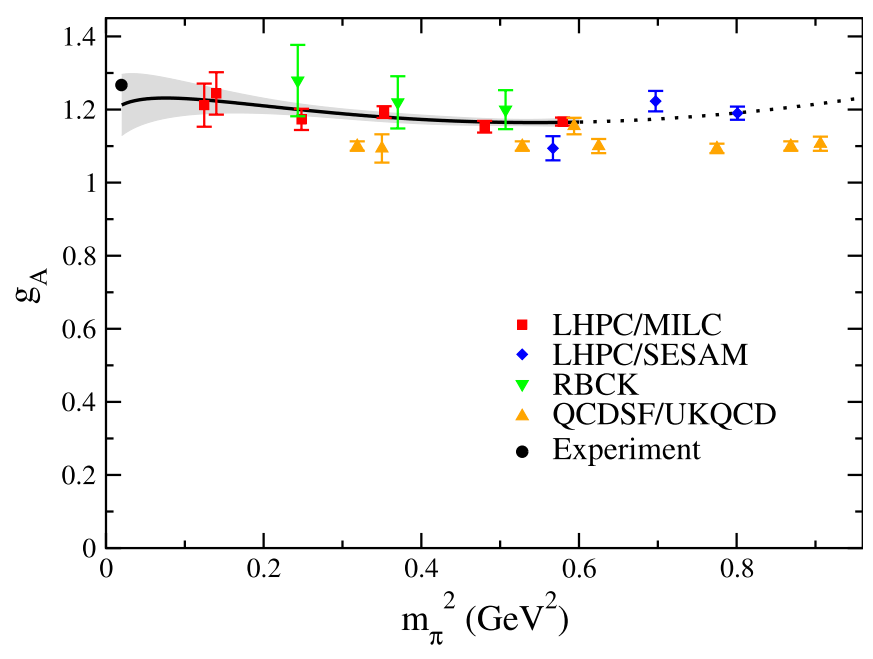

Figure 26. Comparison of all presently existing unquenched lattice QCD calculations of the nucleon's axial charge $g_{\mathrm{A}}$ (LHPC/MILC [160], LHPC/SESAM [161], RBCK [162] and QCDSF/UKQCD [163]). The solid/dotted line and error band denote a chiral perturbation theory fit to the (red) squares only. The error bars reflect only statistical uncertainties and thus the differences between results from different collaborations give an indication of the systematic uncertainty in these calculations. Reprinted with permission from [160]. Copyright (2006) by the American Physical Society.

combination $L_{u}^{\text {latt. }}-L_{d}^{\text {latt. }} \approx-0.4$ is not affected by the omission of disconnected quark loops, and therefore more reliable. Note that in all quark models, where the orbital angular momentum arises from the lower component of a confined relativistic quark wave function, such as the MIT bag model, $u$ quarks have a positive orbital angular momentum, while for $d$ quarks the orbital angular momentum is negative, yielding $L_{u}-L_{d}>0$. Moreover, the sign of the Sivers function, both in SIDIS and in purely hadronic reactions, strongly suggests $L_{u}>L_{d}$. QCD evolution of the quark orbital angular momentum from very low $Q^{2}$, where the quark models are appropriate, to the lattice scale of about $4 \mathrm{GeV}^{2}$ has been suggested as a possible solution for this discrepancy [167]. However, in considering this apparent discrepancy, one should bear in mind that there are different possibilities for defining what is orbital angular momentum, which is discussed in detail in section 5.1 .

Figure 27 shows results for the lowest moment $\int \mathrm{d} x q\left(x, b_{\perp}\right)$ of IPDs. The magnitude of the deformation in these panels (i.e. the deviation from axial symmetry) is a measure for the correlation between the nucleon spin and the quark orbital angular momentum (left) and between the quark spin and quark orbital angular momentum (right). Notice that the average density on the left is twice as large as on the right since both quark polarizations are summed over on the left. The figures clearly indicate that the correlation between quark angular momentum and the nucleon spin (left) has opposite signs for the two quark flavours, while they have the same sign for the correlation with the quark spin (right). Applying the chromodynamic lensing mechanism (section 4.3), this is consistent with opposite sign Sivers functions $f_{1 \mathrm{~T}}^{\perp q}$ (see section 3.6) for $u$ and $d$ quarks. Using the same reasoning for transversely polarized quarks in an unpolarized target, 

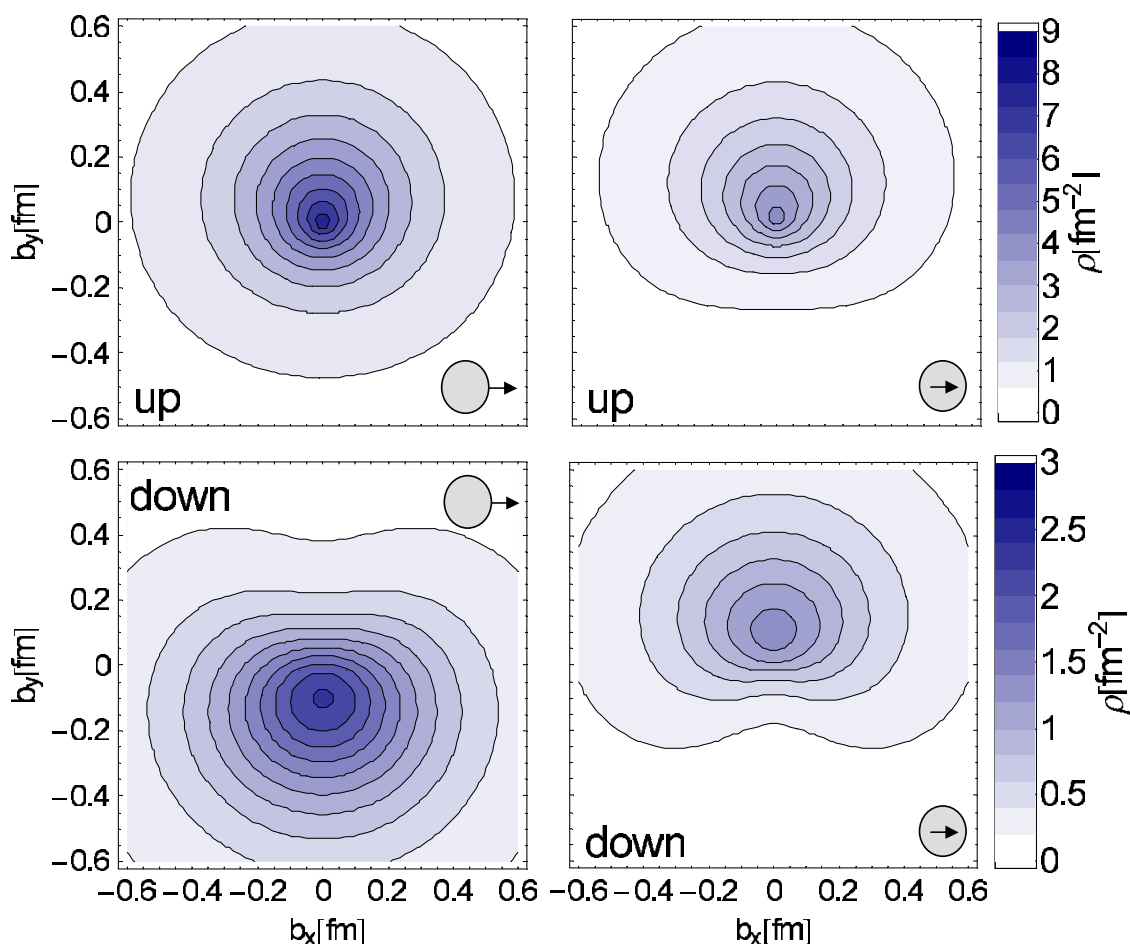

Figure 27. Lowest moment of the IPDs for unpolarized quarks in a transversely polarized nucleon (left) and transversely polarized quarks in an unpolarized nucleon (right) for $u$ (top) and $d$ (bottom) quarks. In the insets, the quark spins (inner arrows) and nucleon spins (outer arrows) are oriented in the transverse plane as indicated. Reprinted with permission from [168]. Copyright (2007) by the American Physical Society.

this leads to same-sign Boer-Mulders functions $h_{1}^{\perp q}$ (see section 3.7) and the sign is the same as the sign of the Sivers function for $u$ quarks (negative). The correlations on the right also appear somewhat stronger than on the left, suggesting $\left|h_{1}^{\perp q}\right|>\left|f_{1 \mathrm{~T}}^{\perp q}\right|$.

Lattice QCD calculations play an important role in complementing both experiments and phenomenological models. For selected hadron structure observables, lattice calculations are reaching a precision that will allow the comparison of QCD predictions to experimental data. Lattice calculations also complement experiments because there are many interesting observables which are difficult to probe experimentally, but which are easily accessible on a lattice. Examples include most chirally odd distribution functions and form factors. Nevertheless, calculations on the lattice will never be able to fully replace experiments in the exploration of hadron structure. For example, no local manifestly gauge invariant operator ${ }^{11}$ exists for $\Delta G$, which is therefore inaccessible in lattice QCD calculations. Simulation of scattering reactions and investigation of unstable particles are enormously difficult. Already the determination of low energy scattering phase shifts requires a sequence of calculations on lattices of various volumes [169-171], thus multiplying the computational time. Only those resonances (unstable particles) the mass of which is close to their decay threshold are accessible by this technique. Furthermore, the necessary use of Euclidean time in lattice calculations makes those observables

${ }^{11}$ An operator that can be written as the same expression in all gauges is called manifestly gauge invariant. inaccessible that require calculations with a real time variable. For those high-energy scattering processes where ISI and/or FSI are essential, such as SIDIS, no useful algorithm has been developed yet and the interpretation of experiments still has to rely on phenomenological models. Even after factorization has been applied in SIDIS, calculating the FSI phase that is crucial for TMDs would again require a real time variable. This is the main reason why no one has found a direct way for calculating the Sivers (22) or Boer-Mulders (24) functions on the lattice. However, ISI/FSI affect the $p_{\mathrm{T}}$ dependence of all PDFs and since the lattice presently can only calculate intrinsic distributions (without ISI/FSI), a direct comparison with experimentally measured $\boldsymbol{p}_{\mathrm{T}}$-dependent distributions (which include ISI/FSI) thus requires modelling of those effects. Likewise, no algorithm exists for calculating fragmentation functions on the lattice.

As a result of these limitations, there has also been a significant synergy between lattice calculations and phenomenological models in recent years. Even though lattice calculations cannot be used for direct calculations in dynamical processes, they can play a useful role in providing phenomenological models with input parameters, thus effectively increasing the predictive power of these models. The above-mentioned predictions for the BoerMulders functions is an excellent example of such a synergy between lattice and phenomenology: in the 'chromodynamic lensing' model for SSAs presented in section 4.3, IPDs are needed as input. For the Boer-Mulders functions, the relevant GPDs are chirally odd and no experimental constraints on them exist, but the lattice calculations of these GPDs [168] leading 


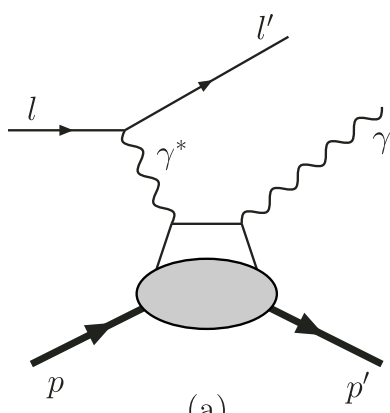

(a)

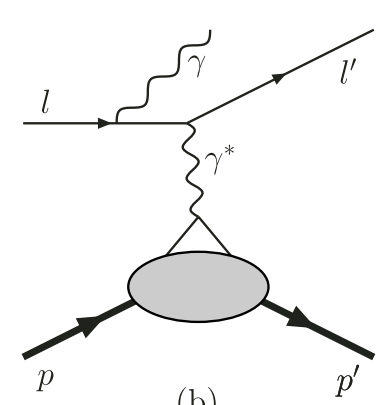

(b)

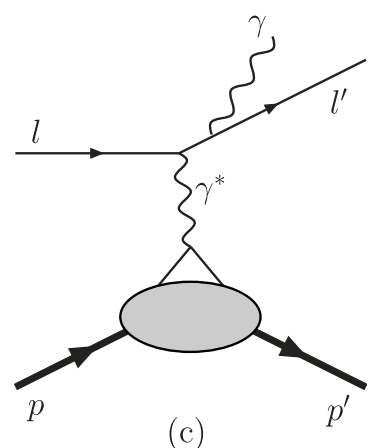

(c)

Figure 28. Leading-order processes for leptoproduction of real photons. Left: DVCS; middle and right: Bethe-Heitler (BH) process.

to the deformations shown in figure 27 allowed predictions to be made for the signs of the Boer-Mulders functions for $u$ and $d$ quarks [126, 172].

\subsection{Access to quark GPDs through exclusive lepton-nucleon scattering}

Presently the experimentally most accessible GPDs are the chirally even quark GPDs $F^{f}(x, \xi, t)(F=H, \widetilde{H}, E, \widetilde{E}$ and $f=u, d)$. In order to constrain their non-forward $(\xi \neq 0)$ behaviour, measurements can be performed of hard exclusive leptoproduction of a photon or meson, leaving the target nucleon intact. Useful observables are cross sections and cross section differences or asymmetries with respect to the polarization of the beam or target, or the beam charge. In these observables, GPDs typically appear convoluted with hard scattering amplitudes, so that they cannot be extracted directly from the measured data. Instead, their functional dependence on $x, \xi$ and $t$ is parametrized in theoretical models (see section 4.6), the parameters of which have to be constrained by comparison with experiment (see section 4.7). For various final states, the convolution integrals appear combined additively or multiplicatively in various ways, with various kinematic prefactors. Hence eventually the extraction of quark GPDs will require a (presently not yet existing) global fit to data from various experiments, as has been customary for decades in the extraction of ordinary PDFs.

4.5.1. Deeply virtual Compton scattering. The exploitation of exclusive production of a real photon, i.e. DVCS ep $\rightarrow$ ep $\gamma$, has two advantages:

(i) among the hard exclusive processes that can be accessed experimentally in the foreseeable future, it is considered to be the one with the most reliable theoretical interpretation;

(ii) effects of NLO [173-175] and subleading twist (see section 1) corrections [176-178] are under theoretical control. For suitably chosen observables the lowest correction is of order $1 / Q^{2}$ instead of $1 / Q$.

In the generalized Bjorken limit of large photon virtuality $Q^{2}$ at fixed $x_{\mathrm{B}}$ and $t$, the dominant pQCD subprocess of DVCS is described by the 'handbag' diagram shown in the left panel of figure 28. The skewness parameter $\xi$ is given by $\xi \simeq x_{\mathrm{B}} /\left(2-x_{\mathrm{B}}\right)$ in this limit. Pictorially, the parton (of flavour $q$ ) taken out of the proton carries the longitudinal momentum fraction $x+\xi$ and the one put back into the proton carries the fraction $x-\xi$. The quark GPD $F^{f}(x, \xi, t)$ can then be considered as describing the correlation between these two quarks at the given value(s) of $t$ (and $Q^{2}$ ).

The $t$ dependence of quark GPDs is directly accessible in DVCS although high experimental precision, i.e. high statistical accuracy in conjunction with sufficient resolution of the experimental apparatus, is required to extrapolate to the limit $t \rightarrow 0$. The latter is of particular importance for the evaluation of the second $x$-moment of the sum of the two 'unpolarized' quark GPDs, $H^{f}+E^{f}$, which is related to the total angular momentum $J_{f}$ of the parton species $f=$ $(u, d, g)$, at a given value of $Q^{2}[19,123]$ (see (37)).

The $\mathrm{BH}$ process, or radiative elastic scattering, is illustrated in the two right panels of figure 28. Its final state is indistinguishable from that of the DVCS process and these two mechanisms have to be combined on the level of the process amplitudes. The differential cross section for leptoproduction of real photons is written in terms of the $\mathrm{BH}$ and DVCS process amplitudes as

$$
\begin{gathered}
\frac{\mathrm{d} \sigma(\mathrm{ep} \rightarrow \mathrm{ep} \gamma)}{\mathrm{d} x_{\mathrm{B}} \mathrm{d} Q^{2} \mathrm{~d}|t| \mathrm{d} \phi} \propto\left|\tau_{\mathrm{BH}}\right|^{2}+\left|\tau_{\mathrm{DVCS}}\right|^{2} \\
+\underbrace{\tau_{\mathrm{DVCS}} \tau_{\mathrm{BH}}^{*}+\tau_{\mathrm{DVCS}}^{*} \tau_{\mathrm{BH}}}_{I} .
\end{gathered}
$$

Here $\phi$ is the azimuthal angle between the scattering plane spanned by the trajectories of the incoming and outgoing leptons and the production plane spanned by the virtual photon and the produced real photon (see figure 29). The $\mathrm{BH}$ process amplitude is calculable using the electromagnetic proton form factors, which are well measured at small $t$ (see, e.g., [179]). The pure DVCS contribution, $\left|\tau_{\mathrm{DVCS}}\right|^{2}$, can then be extracted by integrating over the azimuthal dependence of the cross section. In this situation the interference term $I$ vanishes to leading order in $1 / Q$; its total contribution at collider kinematics was estimated to be at the per cent level [178].

The interference term $I$ is of great interest, as the measurement of its azimuthal dependence opens experimental access to the complex-valued DVCS process amplitude. In this way, both their real and imaginary parts (magnitude and phase) become accessible [180]. This method to study DVCS can be compared with holography [181] in the sense that the phase of the process amplitude of DVCS is measured against the known 'reference phase' of the $\mathrm{BH}$ process. Moreover, at fixed-target 


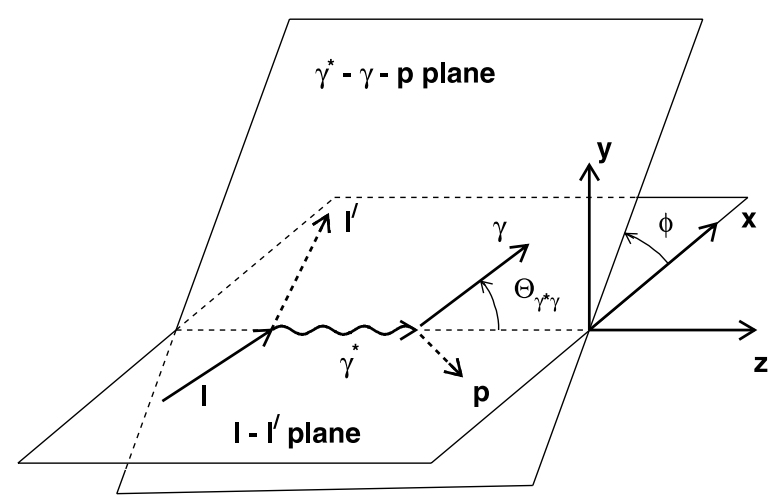

Figure 29. Definition of the azimuthal angle $\phi$ in DVCS in the target rest frame.

energies, the more abundant $\mathrm{BH}$ process can be considered as an 'amplifier' to study the rare DVCS process.

An expansion in $1 / Q$ of the electroproduction cross section (61) reveals distinctive kinematic signatures of DVCS [178, 180,182]. For the interference term $I$ this expansion reads as

$$
\begin{aligned}
I \propto & e_{1}\left[\sum_{n=1,3} k_{n}^{c} \operatorname{Re} \widehat{M}_{n} \cos n \phi\right. \\
& \left.+P_{\text {beam }} \sum_{n=1,2} k_{n}^{s} \operatorname{Im} \widehat{M}_{n} \sin n \phi+\mathcal{O}(1 / Q)\right],
\end{aligned}
$$

where $e_{1}= \pm 1$ is the charge of the lepton beam and the $k_{n}^{c, s}$ are kinematic factors. $\widehat{M}_{n}$ represents linear combinations of helicity (Compton) amplitudes describing the DVCS process. The subscript $n=1,2,3$ labels the helicities for initial and final state photons: $n=1(++)$ means helicity conservation, $n=2(+0)$ single-helicity flip and $n=3(+-)$ doublehelicity flip.

In general, the portion of the interference term containing real parts of Compton amplitudes can be isolated by forming a cross section difference or asymmetry with respect to the charge of the lepton beam [183], while that containing imaginary parts can be accessed by forming single-spin differences or asymmetries with respect to the polarization of the lepton beam [184] or of the target [131,178]. In the interpretation of experimental results in the subsequent sections, always only the helicity-conserving twist-2 Compton amplitude $\widehat{M}_{1} \equiv \widehat{M}_{++}$is considered. In the convention of [178], this amplitude can be written as a linear combination of products of the Dirac and Pauli elastic nucleon form factors $F_{1}$ and $F_{2}$ with the Compton form factors (CFFs) $\mathcal{F}(\mathcal{F}=$ $\mathcal{H}, \mathcal{E}, \widetilde{\mathcal{H}}, \widetilde{\mathcal{E}}$ ). For the three different target polarizations (U for unpolarized, $\mathrm{L}(\mathrm{T})$ for longitudinally (transversely) polarized target) the expressions are

$$
\begin{aligned}
\widehat{M}_{U} & \propto \frac{\sqrt{t_{0}-t}}{2 m}\left[F_{1} \mathcal{H}+\xi\left(F_{1}+F_{2}\right) \tilde{\mathcal{H}}-\frac{t}{4 m^{2}} F_{2} \mathcal{E}\right], \\
\widehat{M}_{L} & \propto \frac{\sqrt{t_{0}-t}}{2 m}\left[\xi\left(F_{1}+F_{2}\right) \mathcal{H}+F_{1} \tilde{\mathcal{H}}\right. \\
& \left.-\left(\frac{\xi}{1+\xi} F_{1}+\frac{t}{4 m^{2}} F_{2}\right)(\xi \widetilde{\mathcal{E}})\right], \\
\widehat{M}_{\mathrm{T}} & \propto \frac{t_{0}-t}{4 m^{2}}\left[F_{2} \mathcal{H}-F_{1} \mathcal{E}+\xi\left(F_{1}+F_{2}\right)(\xi \widetilde{\mathcal{E}})\right],
\end{aligned}
$$

where terms proportional to $\xi^{2}$ and higher are neglected in (64) and (65) [131, 178]. Here $m$ is the nucleon mass and $-t_{0}=4 \xi^{2} m^{2} /\left(1-\xi^{2}\right)$ is the minimum possible value of $-t$ at a given $\xi$.

The complex-valued CFFs $\mathcal{F}\left(\xi, t, Q^{2}\right)$ are flavour sums of convolutions of the corresponding leading-twist quark GPDs with the functions $C_{f}^{\mp}$ describing the hard $\gamma^{*} q$ Compton scattering. The latter are available up to NLO in pQCD [173-175]:

$\mathcal{F}\left(\xi, t, Q^{2}\right)=\sum_{f} \int_{-1}^{1} \mathrm{~d} x C_{f}^{\mp}\left(\xi, x, \log Q^{2}\right) F^{f}\left(x, \xi, t, Q^{2}\right)$.

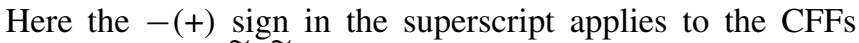
$\mathcal{F}=\mathcal{H}, \mathcal{E}(\widetilde{\mathcal{H}}, \widetilde{\mathcal{E}})$, corresponding to the GPDs $F^{f}=$ $H^{f}, E^{f}\left(\widetilde{H}^{f}, \widetilde{E}^{f}\right)$. At $\mathcal{O}\left(\alpha_{s}\right)$, also contributions from $F^{\mathrm{G}}\left(\tilde{F}^{\mathrm{G}}\right)$ exist in (66) [131]. Except when explicitly needed, the dependence on $Q^{2}$ is omitted in the following.

Only at leading order in $\alpha_{s}$ do there exist relatively simple relationships between the real or imaginary parts of a CFF and (the flavour sum over) the respective quark GPDs that it embodies [178]. Using the same sign convention, the real part of (66),

$\operatorname{Re} \mathcal{F}(\xi, t) \stackrel{\mathrm{LO}}{=} \sum_{f} e_{q}^{2}\left[\mathcal{P} \int_{-1}^{1} \mathrm{~d} x F^{f}(x, \xi, t)\left(\frac{1}{x-\xi} \mp \frac{1}{x+\xi}\right)\right]$,

becomes at given skewness $\xi$ a flavour sum of integrals over $-1<x<1$ of the respective quark GPDs, where $\mathcal{P}$ denotes Cauchy's principal value. The imaginary part of (66),

$\operatorname{Im} \mathcal{F}(\xi, t) \stackrel{\text { LO }}{=} \pi \sum_{f} e_{f}^{2}\left(F^{f}(\xi, \xi, t) \mp F^{f}(-\xi, \xi, t)\right)$,

becomes a direct flavour sum of quark GPD singlet combinations $F^{f}(\xi, \xi, t) \mp F^{f}(-\xi, \xi, t)$. Singlet combinations are also probed in exclusive vector meson production while in exclusive pseudoscalar meson production non-singlet combinations $F^{f}(\xi, \xi, t) \pm F^{f}(-\xi, \xi, t)$ are accessed [131].

A recent theoretical development exploits dispersion relations for CFFs. A CFF $\mathcal{F}$ satisfies a single-variable dispersion relation showing that the entire $\xi$ dependence of the real part is given by a term that can be constructed from only the imaginary part [185-187]

$$
\begin{aligned}
& \operatorname{Re} \mathcal{F}(\xi, t)=\frac{1}{\pi} \mathcal{P} \int_{0}^{1} \mathrm{~d} \xi^{\prime} \operatorname{Im} \mathcal{F}\left(\xi^{\prime}, t\right)\left(\frac{1}{\xi-\xi^{\prime}} \mp \frac{1}{\xi+\xi^{\prime}}\right) \\
& \quad+\mathcal{C}_{\mathcal{F}}(t) .
\end{aligned}
$$

At leading order, the imaginary part depends only on the GPDs on the trajectories $x= \pm \xi$. The $D$-term form factor is then given by

$$
\mathcal{C}_{\mathcal{F}}(t)=\sum_{f} e_{f}^{2} \int_{0}^{1} \mathrm{~d} x D^{f}(x, t)\left(\frac{1}{1-x}\right),
$$

where the $D$-term $D^{f}$ was introduced in (44) of section 4.2. This form factor vanishes for the CFFs $\widetilde{\mathcal{H}}$ and $\widetilde{\mathcal{E}}$ and is identical up to the sign for $\mathcal{H}$ and $\mathcal{E}$ [186, 187]. Equation (69) 
together with (68) imply that at leading order and without considering effects of evolution, the real part of the CFF can be decomposed into a term that shares the well-known property of the imaginary part in depending on the value of the GPDs only along the one-dimensional trajectories $x= \pm \xi$, and another term that depends only on $t$ and $Q^{2}$, and not on $\xi$. Thus, in a leading-order analysis of DVCS data, parametric models for quark GPDs can be constrained only in the subspace $(x= \pm \xi, t)$. Beyond this scenario, a full $(x, \xi)$ mapping of quark GPDs is still possible, at least in principle:

(i) when a large enough range in $Q^{2}$ is covered by DVCS measurements, the known $Q^{2}$-evolution of quark GPDs can be used to constrain their $x$ dependence.

(ii) in hard exclusive leptoproduction of a virtual photon (double DVCS or DDVCS), its virtuality, i.e. the invariant mass of the produced lepton pair, is an additional variable that facilitates a complete mapping of quark GPDs [188, 189]. However, the DDVCS cross section is suppressed by an additional factor $\alpha_{e m}$, thereby making this reaction practically inaccessible using present facilities.

Even if complete DVCS data sets were available for both proton and neutron targets, it would still be a very intricate theoretical problem to deconvolute the quark GPDs $F^{f}$ from the $\mathrm{CFF} \mathcal{F}$. The variable $t$ does not pose any problem as it does not appear in the kernel; i.e. the deconvolution would have to be done at a given value of $t$.

4.5.2. Hard exclusive meson production. Additional information on GPDs is available from hard exclusive leptoproduction of a pseudoscalar or vector meson M: ep $\rightarrow$ epM. To leading order in $1 / Q$ and $\alpha_{s}$, the meson structure itself enters only through a few constants [131]. (In higher order the meson distribution amplitude has to be explicitly taken into account.) The meson production cross section receives contributions from both longitudinal (L) and transverse (T) virtual photons:

$$
\frac{\mathrm{d} \sigma}{\mathrm{d} x_{\mathrm{B}} \mathrm{d} Q^{2} \mathrm{~d}|t|} \propto \sum_{\text {spins }}\left|\mathcal{A}_{\mathrm{T}}\right|^{2}+\epsilon \sum_{\text {spins }}\left|\mathcal{A}_{\mathrm{L}}\right|^{2},
$$

where $\epsilon$ is the ratio of longitudinal and transverse fluxes of the virtual photon. The dependences of the scattering amplitudes $\mathcal{A}$ on the hadron polarizations are summed or averaged over in $\sum_{\text {spins }}$. A factorization theorem has been proved to date only for the longitudinal part [190].

The contribution by transverse photons to the cross section for meson production appears at subleading order in $1 / Q[131,190]$. In the case of production of a vector meson its spin-density matrix can be measured, thus facilitating the longitudinal-transverse separation. In the case of pseudoscalar meson production only data at sufficiently large $Q^{2}$ may be used where the contribution of transverse virtual photons can be neglected.

It appears that for meson production by longitudinal photons, at leading order in $1 / Q$ and for an unpolarized target $[190,131]$, only one pair of quark GPDs is involved in the relevant combination, controlled by the type of spinparity exchange in the meson production, so that by measuring various meson types different pairs of quark GPDs are filtered out. For natural-parity exchange, i.e. for the production of, e.g., vector mesons such as $\rho^{0}$, the quark-helicity-conserving GPDs $H^{f}$ and $E^{f}$ are involved:

$$
\begin{aligned}
& \frac{1}{2} \sum_{\text {spins }}\left|\mathcal{A}_{\mathrm{L}}\right|^{2}=\left(1-\xi^{2}\right)|\mathcal{H}|^{2}-\left(\xi^{2}+\frac{t}{4 m^{2}}\right)|\mathcal{E}|^{2} \\
& \quad-2 \xi^{2} \operatorname{Re}\left(\mathcal{E}^{*} \mathcal{H}\right) .
\end{aligned}
$$

For unnatural-parity exchange, i.e. for the production of, e.g., pseudoscalar mesons such as pions, the quark-helicity-flip GPDs $\widetilde{H}^{f}$ and $\widetilde{E}^{f}$ are involved:

$$
\begin{aligned}
& \frac{1}{2} \sum_{\lambda^{\prime} \lambda}\left|\tilde{\mathcal{A}}_{\mathrm{L}}\right|^{2}=\left(1-\xi^{2}\right)|\tilde{\mathcal{H}}|^{2}-\xi^{2} \frac{t}{4 m^{2}}|\widetilde{\mathcal{E}}|^{2} \\
& -2 \xi^{2} \operatorname{Re}\left(\widetilde{\mathcal{E}}^{*} \tilde{\mathcal{H}}\right) .
\end{aligned}
$$

Recently, detailed numerical studies were presented of the perturbative convergence of the longitudinal cross section for the production of $\rho^{0}, \omega, \phi$ and $\pi^{+}$mesons [191]. At intermediate to large $x_{\mathrm{B}}$, typical for fixed-target experiments, NLO corrections of up to $100 \%$ were found, which somewhat decrease in size when going from $Q^{2}=4$ to $9 \mathrm{GeV}^{2}$. At lower $x_{\mathrm{B}}$, typical for collider kinematics, NLO corrections are huge even for $Q^{2}$ well above $10 \mathrm{GeV}^{2}$. Such an increased sensitivity to higher order corrections is due to the above shown quadratic dependence on GPDs. It also exists in DVCS (see (61)) for the $\left|\tau_{\mathrm{DVCS}}\right|^{2}$ term, but not for the interference term $I$. We note that huge NLO corrections up to 200-300\% appear also in other, mainly collider, processes. Here in most cases the full NLO result is available and used, and typically the NNLO corrections are not again $100 \%$ of the NLO ones.

\subsection{GPD parametrizations}

The application of the Ji relation (37) to evaluate the total angular momentum of quarks of flavour $f$ is a challenging task as it requires, at some fixed common value of $\xi$, knowledge of both $H^{f}$ and $E^{f}$ over the entire $x$ range in the limit of small $t$. In section 4.5.1, it was explained that the best presently practical probe to constrain quark GPDs is DVCS, which unfortunately does not directly provide all of the required information because of its kinematic limitations. While $H^{f}(x, 0,0)=q_{f}(x)$ is well known, $E^{f}(x, \xi, 0)$ is poorly constrained at $\xi=0$, because there it has no influence on experimental observables aside from its first moment, i.e. the Pauli form factor. Hence the Ji relation must be evaluated in the non-forward regime. Furthermore, the integral (37) must be evaluated in the limit $t \rightarrow 0$, even though the crucial dependences of DVCS cross sections on beam charge and polarizations of the beam and target disappear in this limit. Thus GPD parametrizations constrained by DVCS data must be used to extrapolate as $t \rightarrow 0$, which might seem to introduce some degree of arbitrariness. However, GPDs are far from arbitrary functions of the three variables $x, \xi, t$ (or four, when including $Q^{2}$ ), as they must satisfy several relations as described in section 4.2. Considerable ingenuity 
is therefore required in finding analytic representations or phenomenological parametrizations of quark GPDs that embody the constraints of various symmetries and principles, while conveniently parametrizing the remaining degrees of freedom. The discussion on dynamical models, a recent review of which is given in [192], is beyond the scope of this paper.

The simplest ansatz that one might consider for the $t$ dependence of quark GPDs is a 'factorized' form:

$$
H^{f}(x, \xi, t)=h^{f}(x, \xi) F_{1}^{f}(t) .
$$

However, there is evidence from phenomenological considerations [125, 193, 194] and lattice QCD calculations [165, 168] that the $t$ dependence is entangled with both $x$ and $\xi$, so that factorized ansätze are disfavoured. As discussed in section 4.3, $t$ is conjugate to the impact parameter $\boldsymbol{b}_{\perp}$, so that this entanglement corresponds to the interplay between longitudinal and transverse degrees of freedom in the nucleon, sometimes called 'nucleon tomography'. The measured electromagnetic form factors together with some physically motivated functional forms for the limits $x \rightarrow 0,1$ have provided some information about the entanglement of $x$ and $t$ for 'valence' quark flavours in the limited regime $\xi=0$ [193]. In this work, an exponential ansatz was chosen for this entanglement in the valence GPDs:

$$
H^{f(-)}(x, 0, t)=q_{f \mathrm{v}}(x) \exp \left[t f_{f}(x)\right],
$$

where the unpolarized valence PDF $q_{f \mathrm{v}}(x) \equiv q_{f}(x)-\bar{q}_{f}(x)$ is known. Although the dependence on $Q^{2}$ has been suppressed here for convenience, this exponential form is approximately stable under QCD evolution, more so at small $x$. It corresponds to an IPDPDF with guaranteed positivity and a plausible Gaussian shape with an $x$-dependent width:

$$
\begin{aligned}
& q_{f \mathrm{v}}\left(x, \boldsymbol{b}_{\perp}\right)=\frac{1}{4 \pi} \frac{q_{f_{\mathrm{v}}}(x)}{f_{f}(x)} \exp \left[-\frac{\boldsymbol{b}_{\perp}^{2}}{4 f_{f}(x)}\right], \\
&\left\langle\boldsymbol{b}_{\perp}^{2}\right\rangle=4 f_{f}(x) .
\end{aligned}
$$

The choice for the function $f_{f}(x)$ is motivated by simple physical ideas. Partons with small $x$ can be considered to arise from a cascade of branching processes, sometimes called 'Gribov diffusion' [195]. This sort of random walk corresponds to the mean-square impact parameter $\left\langle\boldsymbol{b}_{\perp}^{2}\right\rangle$ growing at small $x$ as $\log (1 / x)$, behaviour that is provided by the 'Regge-motivated' form

$$
\begin{gathered}
H^{f(-)}(x, 0, t) \propto \mathrm{e}^{\left(B_{0} / 2\right) t}\left(\frac{1}{x}\right)^{\alpha^{\prime} t} \cdot q_{f v}(x) \\
=\mathrm{e}^{\alpha^{\prime} t \log (1 / x)+\left(B_{0} / 2\right) t} q_{f \mathrm{v}}(x) .
\end{gathered}
$$

The choice for the function $f_{f}(x)$ is also influenced by plausible expectations for the opposite situation with $x$ approaching unity. As mentioned in section 4.3, a struck quark with large $x$ is expected to have a small impact parameter $\boldsymbol{b}_{\perp}$ with respect to the nucleon centre of momentum given by (55). With the centre of momentum of the spectator collection given by $-b_{\perp} x /(1-x)$, the separation $b_{\perp} /(1-x)$ between quark and spectators can be interpreted as a lower limit on the transverse size of the nucleon, as illustrated in figure 30 . If the size of this confined system is not to diverge as $x \rightarrow 1$, then $\left\langle\boldsymbol{b}_{\perp}^{2}\right\rangle$ must

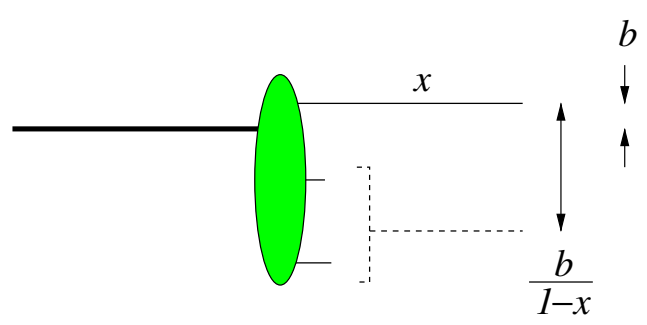

Figure 30. Impact parameter relationship between a struck quark with longitudinal momentum fraction $x$ and the collection of 'spectator' partons. The dashed line indicates the centre of momentum of the spectators and the thick continuous line the centre of momentum of the proton. The figure is taken from [193].

vanish at least as rapidly as $(1-x)^{2}[133,196]$. This implies that $f_{f}(x) \rightarrow A_{f}(1-x)^{n}$ as $x \rightarrow 1$, with $n \geqslant 2$. With $n=2$, this as well as the above constraint for small $x$ are respectively satisfied by the third and first terms in the form

$f_{f}(x)=\alpha^{\prime}(1-x)^{3} \log \frac{1}{x}+B_{f}(1-x)^{3}+A_{f} x(1-x)^{2}$,

where the second term provides a smooth interpolation between them, and $\alpha^{\prime}, A_{f}$ and $B_{f}$ are free parameters to be fitted. Using in (75) and (32) this form for $H^{f(-)}(x, 0, t)$, or a similar form for $E^{f(-)}(x, 0, t)$ together with a simple ansatz for its unknown forward distribution constrained by the known magnetic moments of the proton and neutron, a good fit is obtained of existing data for both Dirac and Pauli elastic form factors of the nucleon. For $x<0.8$, $H^{u(-)}(x, 0, t)$ is well determined, thereby determining the mean-square impact parameter $b_{\perp}$ of $u$ quarks as a function of $x$ in this range. As illustrated in figure 31, there are clear indications that the transverse distribution of $d$ quarks is broader than that of $u$ quarks at large $x$. While the fits for $E^{f(-)}(x, 0, t)$ are ambiguous due to the unknown forward distributions, their second $x$-moments appearing in the $\mathrm{Ji}$ relation (37) are reasonably well determined within the context of the assumed ansatz. It is found that the contributions of the orbital angular momenta of $u$ and $d$ valence quarks to the nucleon spin almost cancel, leaving a small net contribution. In summary, a remarkable amount of information about 'nucleon tomography' was gleaned from only data for nucleon elastic form factors.

In the following, two specific parametric models for quark GPDs that have been most frequently used (in several variants) for comparisons to DVCS data are briefly described as examples. Both are based on quark GPD parametrizations that might, in principle, be employed at any order in $\alpha_{s}$, while the phenomenology has been done at only leading order. The rest of this section has limited goals as a reference tool for continuing students of the field, and might be omitted on a first reading. The first example, the 'VGG model' $[125,198]$, is based on an ansatz that factorizes the dependences on $\xi$ and $t$ in the framework of DDs including the earlier mentioned $D$-term (see section 4.2). Here the flavour dependence of the $D$-term is taken from the chiral quark soliton model [199], resulting in a somewhat modified expression for a generic unpolarized 


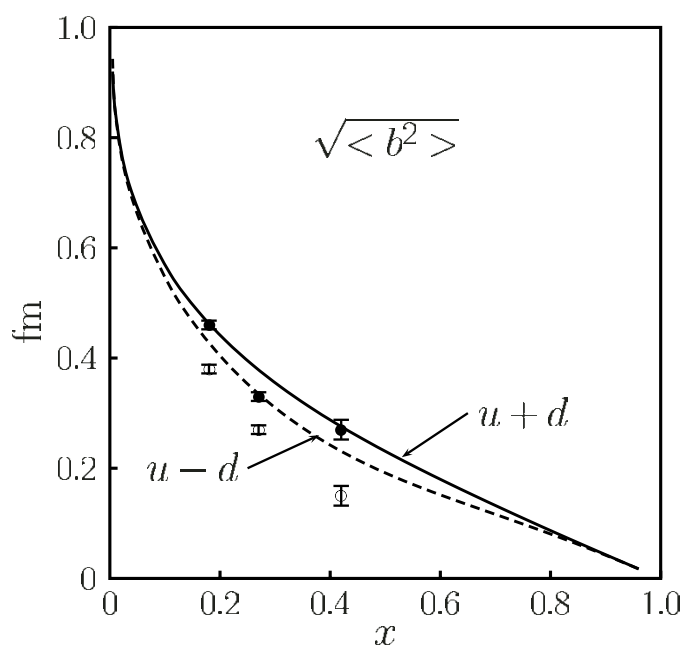

Figure 31. Square root of the mean-square impact parameters $\left\langle b_{\perp}^{2}\right\rangle$ for the sum and the difference of $u$ and $d$ quark distributions from the fit to elastic form factors, compared with lattice QCD results from [197]. The figure is taken from [193].

quark GPD $F(F=H, E)$ :

$$
\begin{aligned}
& F^{f}(x, \xi, t)=\int_{-1}^{1} \mathrm{~d} \beta \int_{-1+|\beta|}^{1-|\beta|} \mathrm{d} \alpha \delta(x-\beta-\xi \alpha) F_{\mathrm{DD}}^{f}(\beta, \alpha, t) \\
& \quad \pm \frac{1}{N_{f}} \theta(\xi-|x|) D\left(\frac{x}{\xi}, t\right)
\end{aligned}
$$

where the upper (lower) sign applies for the GPD $H(E)$. The model ansatz proposed in [200] is invoked to write the DD in (79) as a product

$$
F_{\mathrm{DD}}^{f}(\beta, \alpha, t)=h^{(b)}(\beta, \alpha) F_{\text {forw }}^{f}(\beta, 0, t)
$$

of a 'forward' ( $\xi$-independent) quark GPD $F_{\text {forw }}^{f}(\beta, 0, t)$ with a 'profile function'

$$
h^{(b)}(\beta, \alpha) \propto \frac{\left[1-|\beta|^{2}-\alpha^{2}\right]^{b}}{(1-|\beta|)^{2 b+1}}
$$

that controls the skewness dependence through the (free) profile parameter $b$, with $b \rightarrow \infty$ approaching the forward limits (51) and (52) for $H_{\mathrm{DD}}^{f}(x, 0, t)$ and $\widetilde{H}_{\mathrm{DD}}^{f}(x, 0, t)$, respectively. Often profile parameters are chosen separately for valence and sea quarks. We note that for $b \rightarrow \infty$ this ansatz for $F_{\mathrm{DD}}(x, \xi, t)$ is singular at $x=0$ for finite $\xi$, which is physically implausible. While the clear advantage of the ansatz (80), (81) is its simplicity, it is considered to be too restrictive for flexible modelling of the $(\xi, t)$ dependence of quark GPDs [201,202], especially at small values of $\xi$. The $(x, t)$ dependence is based on either a factorized or the abovementioned Regge-inspired ansatz.

The quark GPD $\widetilde{E}^{f}$ is evaluated from the 'pion pole', which is an ansatz based on the assumption that the dominant contribution at small $|t|$ arises from the emission of a virtual pion by the target nucleon [203,204], which for small $|t|$ is not far off-shell because of the small mass of the pion. The 'pole' in the pion-exchange $t$ dependence $1 /\left(m_{\pi}^{2}-t\right)$ appears in the unphysical region at $t=m_{\pi}^{2}$. This ansatz contributes to only the real part and only in the ERBL kinematic region. Also the pion quantum numbers limit this contribution to only the C-even part of the isovector combination $\widetilde{E}^{u-d}$ and to the corresponding proton-neutron transition GPDs. For the unknown forward (spin-flip) PDF of the GPD $E^{f}$, a parametric combination of valence and sea quark 'PDFs' is used with a functional form inspired by results obtained in [125] using the chiral quark soliton model [205]. In this parametric combination, the quark total angular momenta $J_{f}$ of quarks and antiquarks of flavour $f$ ( $f=u, d$ ) enter as model parameters, so that the derivation of model-dependent constraints on $J_{f}$ becomes possible by comparing predictions with experimental data, as described in section 4.7.6. The VGG model, after its implementation in the 'VGG code' [198], was widely compared with polarized cross sections and asymmetries in beam spin and charge at fixed-target energies and found to describe essential features of the data, especially when using the Regge-inspired ansatz for the $(x, t)$ dependence (see section 4.7.2). As this model inherited the above-mentioned limitations of the adopted DD ansatz, it cannot describe unpolarized cross sections at collider energies where the skewness is small. It was not studied how seriously its limitations may affect the various interpretations of experimental data in terms of this model. For a similar model [178], the effects of NLO corrections to the quark CFFs were found to be significant but moderate, so that they need to be included when extracting model parameters from precise data, while LO calculations were considered to be sufficient for the purpose of estimating observables.

The so-called 'dual-parametrization' model, presented as the second example, is based on the idea of duality in soft hadron-hadron interactions, which is the assumption that the $2 \rightarrow 2$ scattering amplitude in the $s$-channel can be represented as an infinite sum of $t$-channel exchanges [206]. In the case of quark GPDs, this idea is implemented in the form of a $t$-channel partial wave expansion of the Mellin moments [207]. In addition, by using Gegenbauer moments instead of Mellin moments, one can diagonalize the leadingorder QCD evolution kernel, which is a major advantage of this approach. After resumming the partial wave expansion for the Gegenbauer moments, one arrives at an expansion for a quark GPD $F^{f}$ in which each subsequent term is suppressed by two powers of the skewness parameter $\xi$ :

$$
\begin{aligned}
& F^{f}(x, \xi, t)=K_{0} Q_{0}^{f}(x, t)+\xi^{2} K_{2} Q_{2}^{f}(x, t) \\
& \quad+\xi^{4} K_{4} Q_{4}^{f}(x, t)+\cdots .
\end{aligned}
$$

Here $K_{k}$ represents parameter-free $\xi$-dependent (but $t$-independent) integral transformations acting on the variable $x$. The functions $Q_{k}^{f}(x, t)$ are called 'forward-like' functions as their LO evolution is governed by the DGLAP evolution equations, just as for PDFs. The parameters of the model appear in these functions. In the ( $t$-dependent) 'forward' limit $\xi=0$, (82) simplifies to only the first term and $K_{0}$ becomes simple enough to invert. In the case of the quark GPD $H^{f}$, the functions $Q_{0}^{f}(x, t)$ can thus be related to $q_{f}(x, t) \equiv H^{f(+)}(x, 0, t)$ :

$$
\begin{aligned}
& Q_{0}^{f}(x, t)=\left(q_{f}(x, t)+\bar{q}_{f}(x, t)\right) \\
& \quad-\frac{x}{2} \int_{x}^{1} \frac{\mathrm{d} \zeta}{\zeta^{2}}\left(q_{f}(\zeta, t)+\bar{q}_{f}(\zeta, t)\right),
\end{aligned}
$$


and $Q_{0}^{f}(x, 0)$ is thus constrained in terms of the PDFs. An analogous expression holds for the GPDs $E^{f(+)}(x, \xi, t)$ with ( $t$-dependent) 'forward' distributions $e_{f}(x, t)$. The expansion in (82) is introduced in such a way that as $k$ increases their terms are increasingly suppressed for small skewness $\xi$ and fixed $x$. It remains to be shown that this expansion in $\xi$ gives a good approximation for fixed $x / \xi$ when truncated at finite $k$ [208].

The leading-order DVCS amplitude relates to quark GPDs through the convolution integral

$\mathcal{A}^{f}(\xi, t) \stackrel{\mathrm{LO}}{=} \int_{0}^{1} \mathrm{~d} x F^{f(+)}(x, \xi, t)\left[\frac{1}{x-\xi+\mathrm{i} 0}+\frac{1}{x+\xi-\mathrm{i} 0}\right]$.

Another major advantage of the dual parametrization is that this singular integration can be performed analytically in terms of the $Q_{k}^{f}$ [201]:

$$
\begin{gathered}
\operatorname{Im} \mathcal{A}^{f}(\xi, t) \stackrel{\text { LO }}{=}-2 \int_{\bar{x}}^{1} \frac{\mathrm{d} x}{x} N^{f}(x, t) \frac{1}{\sqrt{\frac{2 x}{\xi}-x^{2}-1}}, \\
\operatorname{Re} \mathcal{A}^{f}(\xi, t) \stackrel{\text { LO }}{=}-2 \int_{0}^{\bar{x}} \frac{\mathrm{d} x}{x} N^{f}(x, t)\left[\frac{1}{1-\sqrt{\frac{2 x}{\xi}+x^{2}}}\right. \\
\left.+\frac{1+\sqrt{\frac{2 x}{\xi}+x^{2}}}{+}-\frac{2}{1+x^{2}}\right] \\
-2 \int_{\bar{x}}^{1} \frac{\mathrm{d} x}{x} N^{f}(x, t)\left[\frac{1}{1+\sqrt{\frac{2 x}{\xi}+x^{2}}}-\frac{2}{1+x^{2}}\right.
\end{gathered}
$$$$
-4 \mathcal{D}^{f}(t)
$$

where $\bar{x}=\left(1-\sqrt{1-\xi^{2}}\right) / \xi, N^{f}(x, t) \equiv \sum_{k=0}^{\infty} x^{k} Q_{k}^{f}(x, t)$ and $\mathcal{D}^{f}(t)$ is the $D$-term form factor. The integral transform in (85) can be inverted analytically, yielding

$$
\begin{gathered}
N^{f}(x, t)=\frac{1}{\pi} \frac{x\left(1-x^{2}\right)}{\left(1+x^{2}\right)^{3 / 2}} \int_{x_{0}}^{1} \frac{\mathrm{d} \xi}{\xi^{3 / 2}} \frac{1}{\sqrt{\xi-x_{0}}} \\
\times\left[\frac{1}{2} \operatorname{Im} \mathcal{A}^{f}(\xi, t)-\xi \frac{\mathrm{d}}{\mathrm{d} \xi} \operatorname{Im} \mathcal{A}^{f}(\xi, t)\right],
\end{gathered}
$$

with $x_{0}=2 x /\left(1+x^{2}\right)$. This result can be summarized in the following statements that all hold to leading order only:

- The imaginary part of the DVCS amplitude alone (but for all $\xi$ ) is sufficient to determine $N^{f}(x, t)$. If data are available only above a certain value of $\xi$, one can still reconstruct $N^{f}(x, t)$ above a certain value of $x$. In principle, i.e. given ideal data (negligible uncertainties), this reconstruction can be done exactly.

- Upon inserting $N^{f}(x, t)$ from (87) into (86), data for the real part of the Compton amplitude can be used to determine the $D$-term form factor. Given ideal data with full coverage in $\xi$, the only new information contained in
$\operatorname{Re} \mathcal{A}^{f}(\xi, t)$ that was not already contained in $\operatorname{Im} \mathcal{A}^{f}(\xi, t)$ is the $D$-term form factor. Of course, as ideal data are unfortunately unavailable, (86) also provides useful constraints on $N^{f}(x, t)$ as the $D$-term form factor does not depend on $\xi$.

- At fixed $Q^{2}, N^{f}(x, t)$ and the D-term form factor constitute the only information about quark GPDs that can be obtained from DVCS. However, QCD evolution can be a viable tool for the extraction of quark GPDs, as the LO evolution of the $Q_{2 k}^{f}$ is known to be governed by the LO DGLAP evolution equations. Since the $Q_{2 k}^{f}(x, t)$ enter $N^{f}(x, t)$ multiplied by $x^{2 k}$, the $n$th Mellin moment of $N^{f}(x, t)$ will depend on the $(n+2 k)$ th Mellin moment of the $Q_{2 k}^{f}$. Since the anomalous dimensions that govern the LO evolution depend on the order of the moment (and only on the order), this implies that at different $Q^{2}$ the Mellin moments of $Q_{2 k}^{f}$ (at some renormalization scale $\left.Q_{0}^{2}\right)$ enter $N^{f}(x, t)$ with different relative weights. At least in principle, DVCS data taken at a set of different values of $Q^{2}$ thus provide a system of linear equations for the Mellin moments of the $Q_{2 k}^{f}$ that (after suitable truncation) can be solved, which in turn can be used to reconstruct the quark GPD.

Unfortunately, not only is there a lack of ideal data that are required for the above procedure to work, but also LO evolution is likely to be insufficient at presently accessible values of $Q^{2}$. An approach that is closely related to [201] is that of [209,210], which uses conformal symmetry to diagonalize the evolution up to NLO and may thus overcome the above discussed limitations of the LO treatment.

The dual parametrization does not model the $t$ dependence of the GPDs, which must be specified separately. Present applications of the model do not include the quark GPDs $\widetilde{H}$ and $\widetilde{E}^{f}$ nor higher twist contributions. The model is worked out only to leading-order accuracy, as only then does the DVCS amplitude have a simple form in terms of the $Q_{k}^{f}(t)$, and also their evolution can be handled easily. The generalization from LO to NLO is far from obvious. On the other hand, an advantage of this approach is the clear separation of the ( $\xi$-independent) 'forward' distributions $q_{f}(x, t)\left[e_{f}(x, t)\right]$ of the quark GPDs $H^{f}(x, \xi, t)\left[E^{f}(x, \xi, t)\right]$ from genuine nonforward effects encoded in the distribution of strength among the functions $Q_{2}^{f}, Q_{4}^{f}, \ldots$ in (82). Restricting oneself to the two lowest functions $(k=0,2)$ yields the so-called 'minimal version' of the model [207].

Also assuming in this 'minimal version' $Q_{2}^{f}(x, 0) \propto$ $Q_{0}^{f}(x, 0)$, it was initially found that the $Q^{2}$ and $W$ dependences of the low- $x$ HERA collider data were fairly well described [211], until a missing factor was noticed, leading to a cross section magnitude discrepancy of about a factor of four [212]. In [213], calculations in the minimal version using only the lowest function $Q_{0}^{f}$ (called 'zero-step' approach) as well as calculations of the VGG model were recently compared with JLAB data on cross sections and asymmetries (see section 4.7.3, e.g. figure 39). These comparisons are unaffected by that missing factor.

No global fits to all existing DVCS data yet exist. Future algorithms must necessarily include enough orders beyond LO 

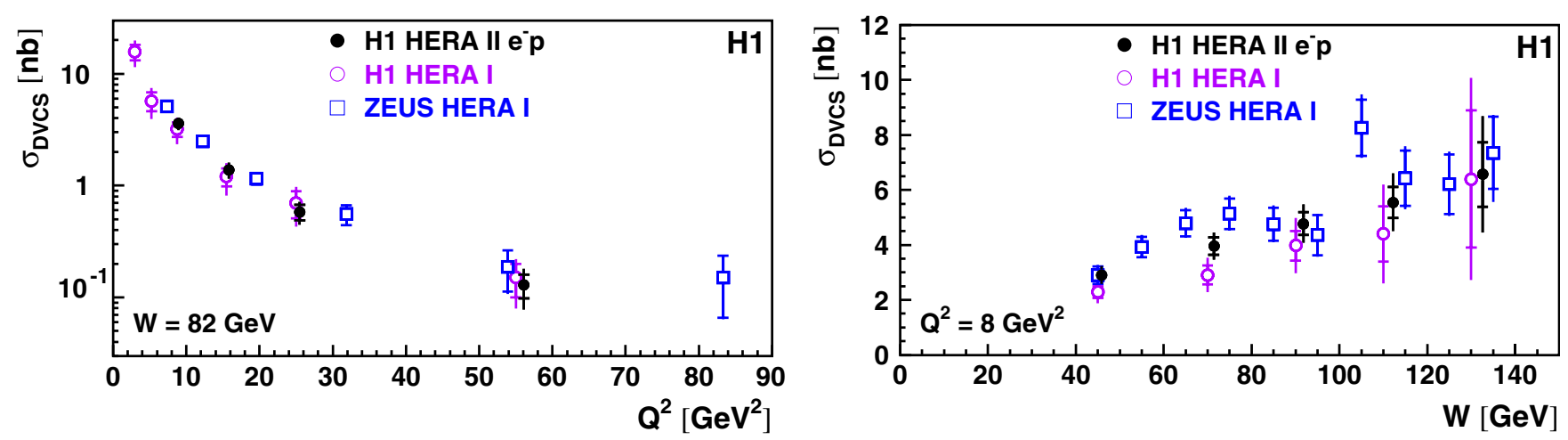

Figure 32. Left: $Q^{2}$ dependence of the differential $\gamma^{*} \mathrm{p} \rightarrow \gamma \mathrm{p}$ cross section measured by $\mathrm{H} 1$ and Zeus. Right: as left panel, but here the $W$ dependence is shown. Reprinted from [215], copyright (2008), with permission from Elsevier.

to ensure convergence. The only existing approach fulfilling this requirement is a recently developed formalism [186] based on dispersion relations discussed in section 4.5.1. In this new formalism, dispersion relation and operator expansion techniques are combined leading to convergence of the conformal partial wave expansion of the VCS amplitude. This formalism was successfully applied to fit DVCS observables in NNLO at low $x_{\mathrm{B}}$ [186], as described in section 4.7.1. In a first attempt, this fit was very recently extended to include also data from fixed-target experiments [214].

\subsection{DVCS experimental results}

4.7.1. DVCS cross section at high energies. The DVCS cross section (61) integrated over its azimuthal dependence has been measured in hard exclusive electroproduction of photons by the experiments $\mathrm{H} 1$ and Zeus at the Hera collider. For the $x_{\mathrm{B}}$ range accessible at collider kinematics $\left(\mathcal{O}\left(10^{-2}-10^{-4}\right)\right)$, twogluon exchange plays a major role besides the quark-exchange handbag diagram of figure 28 , so that both gluon and quark GPDs are probed simultaneously. This is, however, limited to small skewness values below $10^{-2}$.

The analysis method used by the two experiments is similar due to their similar geometries. As the outgoing proton remains undetected in the beam pipe, the event topology is defined by two electromagnetic clusters, the outgoing lepton and the produced real photon, at most one of which is associated with a charged track. Two event samples are selected:

(i) in the 'DVCS-enriched' sample an energetic photon is required. With respect to the incoming lepton it is required to be detected at a large scattering angle while the outgoing lepton is measured at a small angle. Still, a high enough virtuality $Q^{2}>4 \mathrm{GeV}^{2}$ is ensured by requiring a large energy of the scattered lepton $(>15 \mathrm{GeV})$.

(ii) in the $\mathrm{BH}$ dominated 'reference sample', the radiatively produced photon is emitted at a small angle with respect to the incoming lepton, and the outgoing lepton at a large angle.

A Monte Carlo simulation of the completely known $\mathrm{BH}$ process, validated by the reference sample, is used to subtract the $\mathrm{BH}$ contribution from the DVCS-enriched sample. The remainder of the spectrum is due to DVCS and possible additional background; no contribution from the interference term exists at leading twist, as the data are integrated over the azimuthal angle.

The $Q^{2}$ dependence of the differential $\gamma^{*} \mathrm{p} \rightarrow \gamma \mathrm{p}$ cross section is shown in the left panel of figure 32, extracted from Hera-I and Hera-II data of H1 [215] and the Hera-I data of ZEUs [216], the latter based on a somewhat larger event sample. The corresponding $W$ dependence, where $W$ is the invariant mass of the system of virtual photon and proton, is displayed in the right panel. The virtuality appears high enough to assign the observed rise with $W$ to the nature of DVCS as a hard process, as increasing $W$ implies decreasing $x_{\mathrm{B}}$, where the parton densities in the proton show a rapid rise.

In figure 33, various colour dipole model calculations [218-220] are compared with only the HERA-I data of both experiments. Presently it is impossible to discriminate between the various model versions.

A commonly used ansatz for the $t$ dependence of quark GPDs is an exponential decrease $\mathrm{e}^{-b|t|}$. The slope parameter $b$ was first measured in DVCS by H1 [215], the results being shown in the two left panels of figure 34 for $\left\langle Q^{2}\right\rangle=8,15.5$ and $25 \mathrm{GeV}^{2}$ integrated over $W$, and for $\langle W\rangle=40,70$ and $100 \mathrm{GeV}$ integrated over $Q^{2}$. Parametrizing a possible $Q^{2}$ dependence as $b\left(Q^{2}\right)=A\left(1-B \cdot \log \left(Q^{2} / 2 \mathrm{GeV}^{2}\right)\right)$, the fit shows that it is indeed significant with $A=6.98 \pm 0.54 \mathrm{GeV}^{2}$ and $B=0.12 \pm 0.03$. At $Q^{2}=8 \mathrm{GeV}^{2}$ the result is $b=5.45 \pm 0.19_{\text {stat }} \pm 0.34_{\text {syst }}$. Following [135, 221], this $t$ slope can be converted to an average impact parameter which describes the transverse extension of partons. The $\mathrm{H} 1 \mathrm{result}$ at $\langle x\rangle=1.2 \times 10^{-3}$, where sea quarks and gluons dominate, is $\sqrt{\left\langle r_{\mathrm{T}}^{2}\right\rangle}=0.65 \pm 0.02 \mathrm{fm}$. This can be compared with the transverse charge radius of the proton [222]:

$\sqrt{\left\langle r_{\mathrm{T}}^{2}\right\rangle_{\mathrm{ch}}} \equiv \sqrt{-\left.4 \frac{\mathrm{d}}{\mathrm{d} Q^{2}} F_{1}\left(Q^{2}\right)\right|_{Q^{2}=0}}=0.65 \pm 0.01 \mathrm{fm}$.

Recently, the convergence of the perturbative treatment of DVCS was studied in the low- $\xi$ region typical for collider kinematic conditions, in a theoretical study using the predictive power of conformal symmetry [223]. Beyond NLO, further terms in the perturbation series are found to be small for an input scale of a few $\mathrm{GeV}^{2}$. Concerning the scale dependence, 

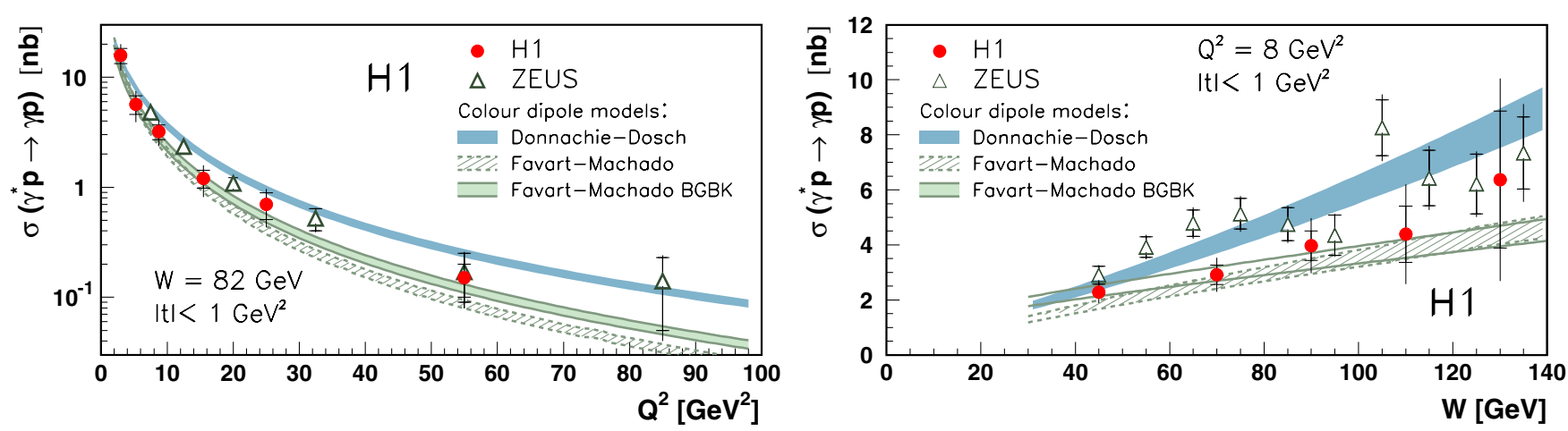

Figure 33. Hera-I data shown in the previous figure in comparison to various colour dipole model calculations (see text). The figures are taken from [217].

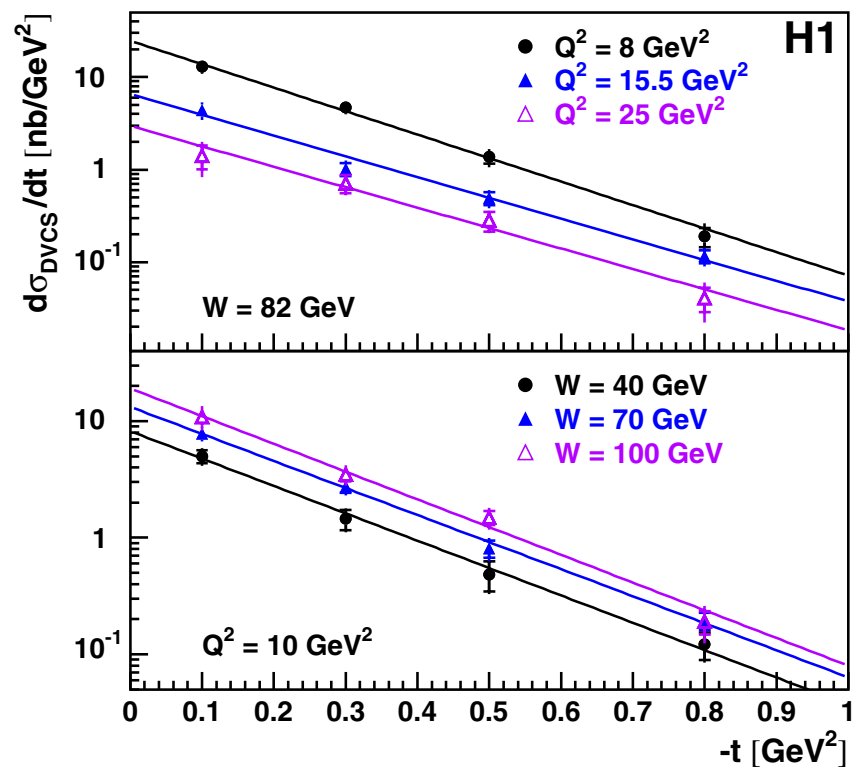

Figure 34. The $-t$ dependence of the differential $\gamma^{*} \mathrm{p} \rightarrow \gamma \mathrm{p}$ cross section, extracted from H1 HeRA II data at 3 values of $\left\langle Q^{2}\right\rangle$. Reprinted from [215], copyright (2008), with permission from Elsevier.

larger corrections due to evolution may appear only for values of $\xi$ below $5 \times 10^{-3}$, similar to the situation in DIS [224].

Based on this approach, very recently a simultaneous fit was performed to the DVCS and DIS collider data, for the first time up to NNLO accuracy [186]. The $\overline{C S}$ scheme was used which differs from the more commonly used $\overline{\mathrm{MS}}$ scheme only in the skewness dependence of the conformal moments in the Mellin-Barnes representation, the latter being used to evaluate the CFFs. In figure 35 the fit results are compared with the data, showing fair agreement. It is argued in [186] that this should be considered satisfactory, as several aspects of the theoretical approach are not completely settled yet, in particular regarding the skewness dependence of quarks GPDs.

4.7.2. DVCS cross sections at low energies. The helicitydependent DVCS cross sections $\mathrm{d}^{4} \sigma^{--\rightarrow} / \mathrm{d} Q^{2} \mathrm{~d} x_{\mathrm{B}} \mathrm{d} t \mathrm{~d} \phi$ and $\mathrm{d}^{4} \sigma^{+--} / \mathrm{d} Q^{2} \mathrm{~d} x_{\mathrm{B}} \mathrm{d} t \mathrm{~d} \phi$ were measured at JLAB with $5.75 \mathrm{GeV}$ electron beams of positive and negative helicity, respectively [226]. Here the symbol $\rightarrow-\rightarrow(\leftarrow--)$ designates positive (negative) beam helicity. In a three-arm kinematically complete experiment, the scattered electron was measured by the high-resolution spectrometer, the produced real photon by an electromagnetic calorimeter and the recoil proton by a scintillator array. Three photon virtualities were selected with $x_{\mathrm{B}}=0.36$ fixed: $Q^{2}=1.5,1.9,2.3 \mathrm{GeV}^{2}$. For each of them, four $-t$ values were chosen: $0.17,0.23,0.28,0.33 \mathrm{GeV}$.

From the two measured single-helicity cross sections, the polarized and unpolarized DVCS cross sections were obtained:

$$
\begin{aligned}
& \frac{\mathrm{d}^{4} \Sigma}{\mathrm{d} Q^{2} \mathrm{~d} x_{\mathrm{B}} \mathrm{d} t \mathrm{~d} \phi}=\frac{1}{2}\left(\frac{\mathrm{d}^{4} \sigma^{--\rightarrow}}{\mathrm{d} Q^{2} \mathrm{~d} x_{\mathrm{B}} \mathrm{d} t \mathrm{~d} \phi}-\frac{\mathrm{d}^{4} \sigma^{+--}}{\mathrm{d} Q^{2} \mathrm{~d} x_{\mathrm{B}} \mathrm{d} t \mathrm{~d} \phi}\right) \\
& \quad \propto \operatorname{Im} I, \\
& \frac{\mathrm{d}^{4} \sigma}{\mathrm{d} Q^{2} \mathrm{~d} x_{\mathrm{B}} \mathrm{d} t \mathrm{~d} \phi}=\frac{1}{2}\left(\frac{\mathrm{d}^{4} \sigma^{--\rightarrow}}{\mathrm{d} Q^{2} \mathrm{~d} x_{\mathrm{B}} \mathrm{d} t \mathrm{~d} \phi}+\frac{\mathrm{d}^{4} \sigma^{+-}}{\mathrm{d} Q^{2} \mathrm{~d} x_{\mathrm{B}} \mathrm{d} t \mathrm{~d} \phi}\right) \\
& \quad \propto \operatorname{Re} I,
\end{aligned}
$$

where $I$ is the interference term that consists of linear combinations of CFFs. Cross section measurements involve additional experimental systematics, as no cancellations of systematic uncertainties occur as in asymmetry measurements. Such measurements have the advantage that the cross sections in (89) and (90) are directly proportional to $\operatorname{Im} I$ and $\operatorname{Re} I$, respectively, while in the case of asymmetries unwanted $\mathrm{BH}$ terms appear in the denominator.

The data for the polarized and unpolarized DVCS cross sections are shown in figure 36 together with Fourier decompositions according to (62). The polarized cross section exhibits the expected $\sin \phi$ behaviour. For the unpolarized one, the main contributions stem from the $|\mathrm{BH}|^{2}$ term and the twist-2 part of the interference term, while $|\mathrm{DVCS}|^{2}$ and twist-3 contributions are found to be negligible.

4.7.3. Beam-spin asymmetry (BSA). It was shown in section 4.5 that in hard exclusive leptoproduction of a real photon, the interference of the $\mathrm{BH}$ and DVCS processes allows the extraction of a wealth of information on quark GPDs. For the case of an unpolarized (U) proton target, the BSA for a longitudinally $(\mathrm{L})$ polarized beam is given by

$$
\mathcal{A}_{\mathrm{LU}}(\phi)=\frac{\mathrm{d} \sigma^{--\rightarrow}(\phi)-\mathrm{d} \sigma^{+--}(\phi)}{\mathrm{d} \sigma^{--\rightarrow}(\phi)+\mathrm{d} \sigma^{+-}(\phi)} .
$$



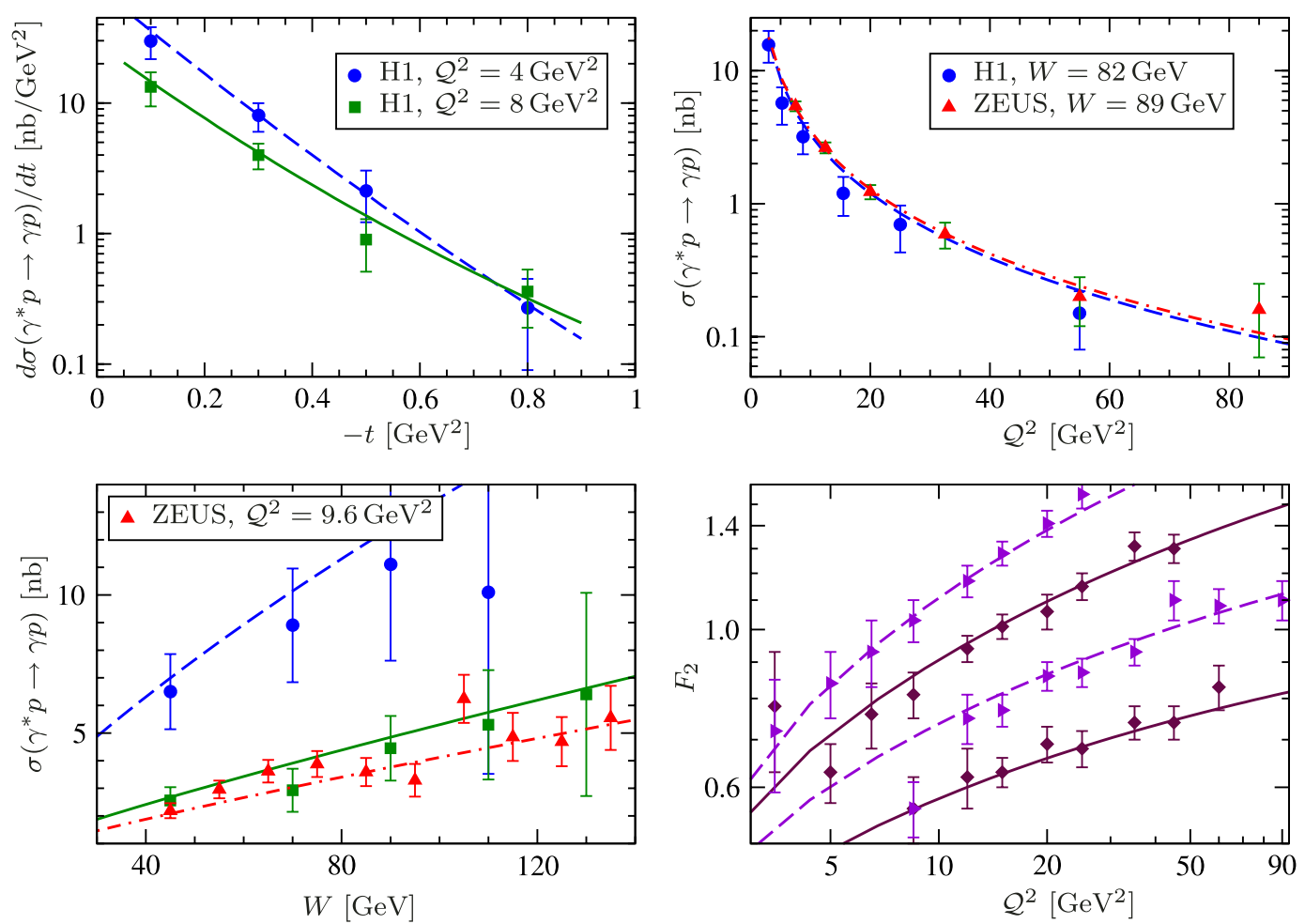

Figure 35. Simultaneous fit to the DVCS and DIS data in the $\overline{\mathrm{CS}}$ scheme at NNLO. Upper left panel: DVCS cross section for $Q^{2}=4 \mathrm{GeV}^{2}$ and $W=71 \mathrm{GeV}$ (circles, dashed line) as well as $Q^{2}=8 \mathrm{GeV}^{2}$ and $W=82 \mathrm{GeV}$ (squares, solid line) [217]. Upper right panel: DVCS cross section $\left(-t<-1 \mathrm{GeV}^{2}\right)$ versus $Q^{2}$ for $W=82 \mathrm{GeV}$ (H1, circles, dashed line) and $W=89 \mathrm{GeV}$ (ZEUS, triangles, dash-dotted line) [216]. Lower left panel: DVCS cross section versus $W$ for $Q^{2}=4 \mathrm{GeV}^{2}$ (H1, circles, dashed line), $Q^{2}=8 \mathrm{GeV}^{2}$ (H1, squares, solid line) and $Q^{2}=9.6 \mathrm{GeV}^{2}$ (ZEUS, triangles, dash-dotted line). Lower right panel: $F_{2}\left(x_{\mathrm{B}}, Q^{2}\right)$ versus $Q^{2}$ for $x_{\mathrm{B}}=8 \times 10^{-3}, 3.2 \times 10^{-3}, 1.3 \times 10^{-3}, 5 \times 10^{-4}$ [225]. Reprinted from [186], copyright (2008), with permission from Elsevier.

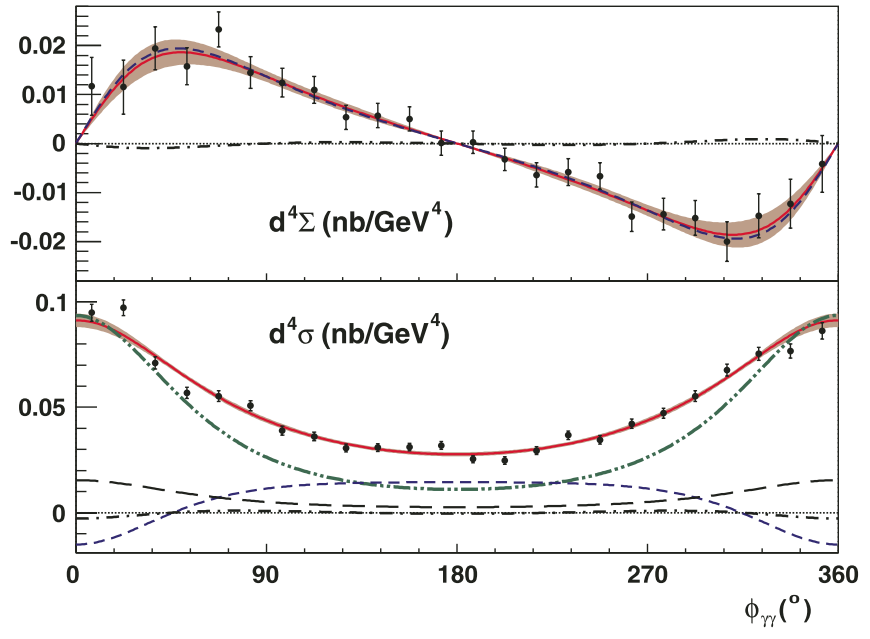

Figure 36. JLAB data and fit to $\mathrm{d}^{4} \Sigma / \mathrm{d} Q^{2} \mathrm{~d} x_{\mathrm{B}} \mathrm{d} t \mathrm{~d} \phi$ and $\mathrm{d}^{4} \sigma / \mathrm{d} Q^{2} \mathrm{~d} x_{\mathrm{B}} \mathrm{d} t \mathrm{~d} \phi$, shown as a function of the azimuthal angle $\phi$ at $Q^{2}=2.3,-t=-0.28$ at $\left\langle x_{\mathrm{B}}\right\rangle=0.36$. Only statistical errors are shown. The solid line shows the total fit with the band representing one standard deviation. The dot-dot-dashed line is the $|\mathrm{BH}|^{2}$ squared contribution, twist-2 (twist-3) interaction terms are shown by a long-dashed (dot-dashed) line. Reprinted with permission from [226]. Copyright (2006) by the American Physical Society.

Here $-\rightarrow(\leftarrow--)$ denotes beam spin parallel (antiparallel) to the beam direction.

The interference term $I$ can be Fourier expanded in the azimuthal angle $\phi$. Evaluating the above asymmetries to leading power in $1 / Q$ in each contribution to this expansion and to leading order in $\alpha_{s}$, only the $\sin \phi(\cos \phi)$ term remains in the numerator of the beam-spin (beam-charge) asymmetry. To the extent that the leading $\mathrm{BH}$-term dominates the denominator, the azimuthal dependence of the beam-spin (beam-charge) asymmetry is reduced to $\sin \phi(\cos \phi)$ :

$$
\mathcal{A}_{\mathrm{LU}}(\phi) \propto \operatorname{Im} \widehat{M}_{\mathrm{U}} \sin \phi .
$$

The BSA is sensitive to the linear combination $\widehat{M}_{\mathrm{U}}$ of CFFs, which describes an unpolarized proton target [178] (see (63)). Generally, the quark GPDs $H^{f}$ are expected to dominate expression (63), because (i) the second term is suppressed by at least a factor of 10 , as $\xi$ is usually not larger than 0.2 even in fixed-target kinematics, and the unpolarized contribution $\mathcal{H}$ is expected to dominate the polarized one $\widetilde{\mathcal{H}}$, in analogy to the forward case; (ii) the third term is $t$-suppressed, by about a factor of 25 for typical $t$-values of about $0.15 \mathrm{GeV}^{2}$. For scattering on the proton, the quark GPD $H^{u}$ will yield the major contribution to $\widehat{M}_{\mathrm{U}}$ because $u$ quarks (i) are more abundant in the proton and (ii) more readily absorb virtual photons because of their four times larger charge-squared factor.

The first published GPD-related experimental results were beam-spin asymmetries measured in DVCS on the proton by the fixed-target experiments HERMES at HERA [227] with a positron beam and by CLAS at Jefferson Laboratory [228] with an electron beam (see figure 37). Note that opposite 

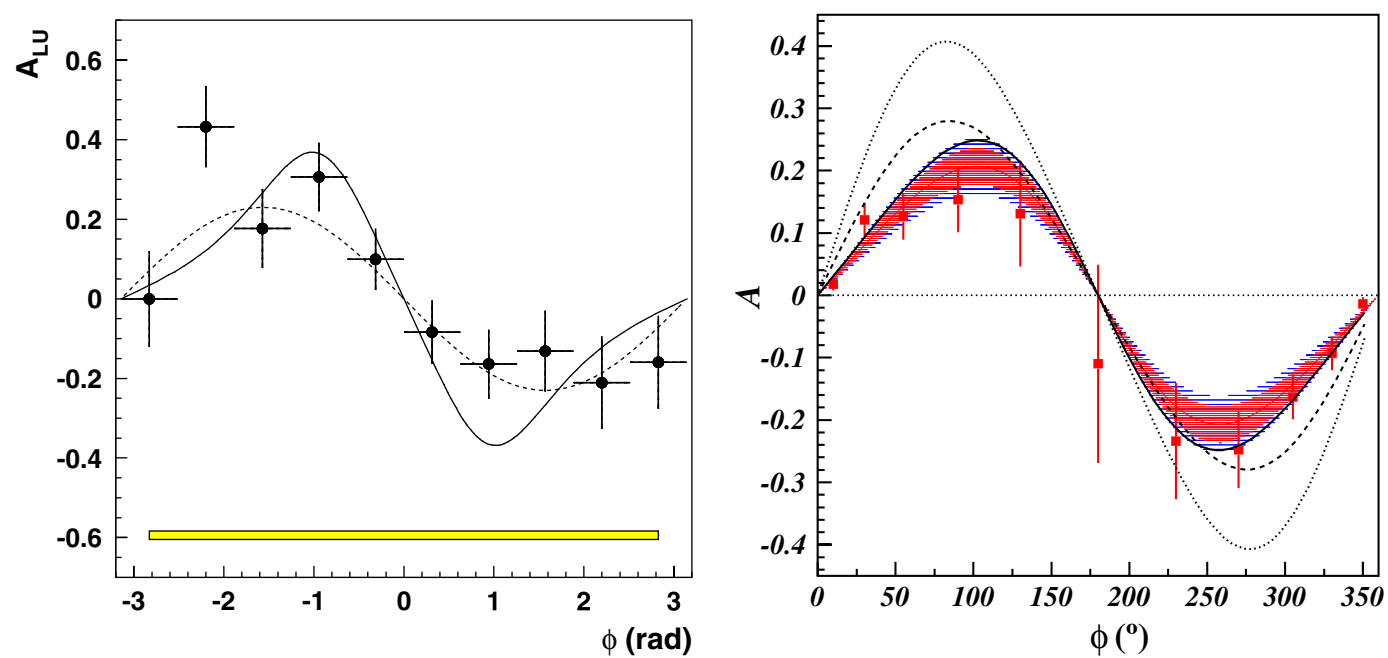

Figure 37. Azimuthal dependence of the BSA. Left: Hermes proton data taken with $27.6 \mathrm{GeV}$ positrons. Right: Clas proton data taken with 4.25 GeV electrons. Reprinted figures with permission from [227, 228]. Copyright (2001) by the American Physical Society.

beam charges imply opposite signs of the measured beam spin asymmetries (this is not immediately apparent in figure 37 because of different $\phi$-ranges shown for Hermes and Clas). In both experiments significant sinusoidal modulations have been observed, as expected from the theoretical considerations that were briefly explained above.

DVCS on the proton was recently measured at JLAB with a $5.77 \mathrm{GeV}$ polarized electron beam in a kinematically complete experiment using the large-acceptance CLAS spectrometer. BSA results were shown in a simultaneous binning in $x, t$ and $Q^{2}$ (62 bins in total) (see figure 38 [229]). The background from undetected exclusive $\pi^{0} \mathrm{~s}$ decaying asymmetrically was estimated using measured symmetric decays and their relative acceptance function determined by Monte Carlo techniques. It was subtracted in every kinematic bin, over which it varies from $1 \%$ to $25 \%$ (5\% on average). Calculations using the earlier described VGG model including only the contribution of the quark GPD $H$, shown as two neighbouring lines, solid for twist-2 and dashed for twist-3, clearly overshoot most of the data. Calculations in a Regge model [230], shown for comparison in a few panels as the lower dashed curve, describe the data well in certain bins but not in others.

For a few particular bins, the azimuthal dependence of the same data was compared in [213] with the VGG and the dual-parametrization models that were described in section 4.6. For both models, the same unfactorized ansatz (77) was used. For simplicity, only the BSA is shown in figure 39, although in [213] also the polarized and unpolarized cross sections are discussed. The VGG calculation, for which $b=1$ was used as profile parameter, overshoots the data as already discussed in connection with the previous figure. The dual-parametrization calculation, done with only the forward function $Q_{0}$, also overshoots the data, although less severely. Adding some estimate for the non-forward function $Q_{2}$ does not improve the situation. Using the values $0, \pm \frac{4}{3}$ for the $D$-term form factor $\left.\mathcal{D}(t)\right|_{t=0}$ that was mentioned after (87) does not yield a simultaneous consistent description of the $\phi$ dependence of the unpolarized and polarized cross sections and the BSA [213]. Such a description cannot be achieved by any of the presently existing GPD models. A similar conclusion is reached in [231] after attempting a combined fit of the two JLAB data sets [226, 229] to variants of the VGG model. It has not been tried to estimate the possible influence of higher order effects for either of the two models.

4.7.4. Beam-charge asymmetry (BCA). The measurement of a BCA

$$
\mathcal{A}_{\mathrm{C}}(\phi)=\frac{\mathrm{d} \sigma^{+}(\phi)-\mathrm{d} \sigma^{-}(\phi)}{\mathrm{d} \sigma^{+}(\phi)+\mathrm{d} \sigma^{-}(\phi)},
$$

where the superscripts + and - denote the lepton beam charge, requires data for both beam charges. Alternatively, a BCA can also be derived from the $\cos \phi$ dependence of the cross section for one beam charge in an hermetic detector of known efficiency. Using the same approximations as in the previous section, the azimuthal dependence of the BCA is reduced to $\cos \phi$ :

$$
\mathcal{A}_{\mathrm{C}}(\phi) \propto \operatorname{Re} \widehat{M}_{\mathrm{U}} \cos \phi .
$$

The BCA is sensitive to the real part of the same linear combination $\widehat{M}_{U}$ (63), of which the imaginary part determines the BSA described in the previous section.

HerA was the only multi-GeV accelerator providing both electron and positron beams. It offered the additional flexibility of inverting every few weeks, for the same charge of the beam, the direction of its polarization to reduce systematic effects. The first measurement of a BCA was published by HeRmes [232], based on the analysis of parts of their electron and positron beam data sets. Somewhat later, BCA results were published based on the analysis of another much larger HERMES data sample, taken with transverse target polarization [233]. Here, beam-charge and transverse-target-spin asymmetries were extracted simultaneously (see section 4.7.6). In figure 40, the experimental data on the azimuthal asymmetry amplitude $A_{C}^{\cos \phi}$ are compared with calculations using the model of $[125,198]$ that is briefly described in section 4.6. The model results are calculated at HERMEs kinematics, each at the average kinematics of each individual bin. The best description 


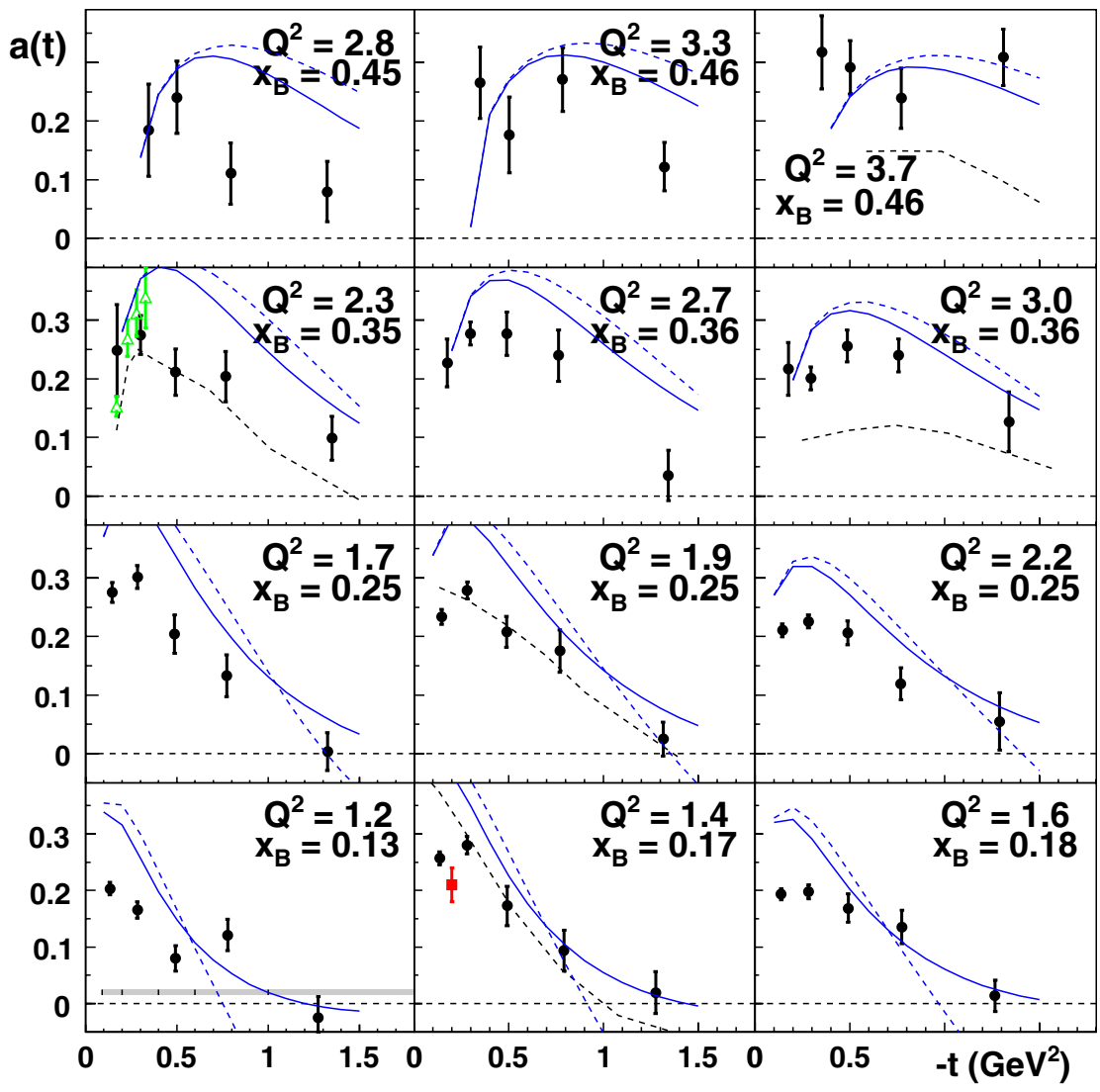

Figure 38. BSA measured at JLAB as a function of $t$ and shown at $\phi=90^{\circ}$ in several $\left(x_{\mathrm{B}}, Q^{2}\right)$ bins. Systematic uncertainties and bin limits are shown by the (grey) band in the lowest left panel. (Black) circles are from [229], the (red) square is earlier data [228] and the open (green) triangles are the cross section data [226] described in the previous section. The curves are described in the text. Reprinted with permission from [229]. Copyright (2008) by the American Physical Society.

within the VGG model is given by the version using an unfactorized Regge-inspired $t$ dependence, no $D$-term and profile parameters $b_{\mathrm{val}}=\infty$ (no skewing) and $b_{\text {sea }}=1$ (some skewing).

4.7.5. Longitudinal target-spin asymmetry. Experimental access to quark GPDs other than $H^{f}$ becomes possible when using an unpolarized beam (U) and a polarized target. For longitudinal (L) target polarization, the same sin $\phi$ dependence is expected as for the BSA, although the relevant twist-2 Compton amplitude $\widehat{M}_{\mathrm{L}}(64)$ involves a different combination of CFFs than does $\widehat{M}_{\mathrm{U}}$ (63).

The longitudinal target-spin asymmetry is defined as:

$$
\mathcal{A}_{\mathrm{UL}}(\phi)=\frac{\mathrm{d} \sigma^{\digamma}(\phi)-\mathrm{d} \sigma^{\Rightarrow}(\phi)}{\mathrm{d} \sigma^{\digamma}(\phi)+\mathrm{d} \sigma^{\Rightarrow}(\phi)},
$$

where $\Leftarrow(\Rightarrow)$ denotes target spin antiparallel (parallel) to the beam direction. This asymmetry is expected to be most sensitive to a combination of $H^{f}$ and $\widetilde{H}^{f}$, because the kinematic suppression of the first term in (64), relative to the second, may compensate the usual dominance of the unpolarized quark GPDs $H^{f}$ over their polarized counterparts $\widetilde{H}^{f}$. Hence they might be disentangled by combining this measurement with BSAs. For not too small values of $t$, there exists also some sensitivity to $(\xi \widetilde{\mathcal{E}})$, which is written in this way because $\widetilde{\mathcal{E}}$ itself is defined to be inversely proportional to $\xi$ (see e.g. [187]).

Experimental results on longitudinal target-spin asymmetries were obtained at JLAB [234] and HeRmes [235]. Both experiments observe a $\sin \phi$ modulation of the yield from the entire acceptance, and scaling of the sinusoidal amplitude with $\sqrt{-t}$, in agreement with expectations. In figure 41, the JLAB data [234] are shown. The continuous curve represents a fit with the function $\alpha \sin \phi+\beta \sin 2 \phi$ with the parameters $\alpha=0.252 \pm 0.042$ (stat) \pm 0.020 (syst) and $\beta=$ $-0.022 \pm 0.045$ (stat) \pm 0.021 (syst). The $\sin 2 \phi$ term is consistent with zero, indicating that higher twist contributions in this kinematic domain are negligible. The two other curves show calculations using the DD model of [198], based on MRST02 PDFs, with the $\xi$ dependence $b_{\text {val }}=b_{\text {sea }}=1$, including leading-twist terms only, and target-mass corrections applied. The nucleon-spin-flip quark GPDs $E$ and $\widetilde{E}$ are set to zero. The dashed (dotted) line shows the asymmetry when the polarized quark GPD $\widetilde{H}$ is included (excluded), so that the necessity of $\widetilde{H}$ for the description of the longitudinally target-spin asymmetry is clearly demonstrated.

4.7.6. Transverse target-spin asymmetry. In the case of target polarization transverse (T) to the direction of the virtual photon, the polarization can be resolved into two independent 

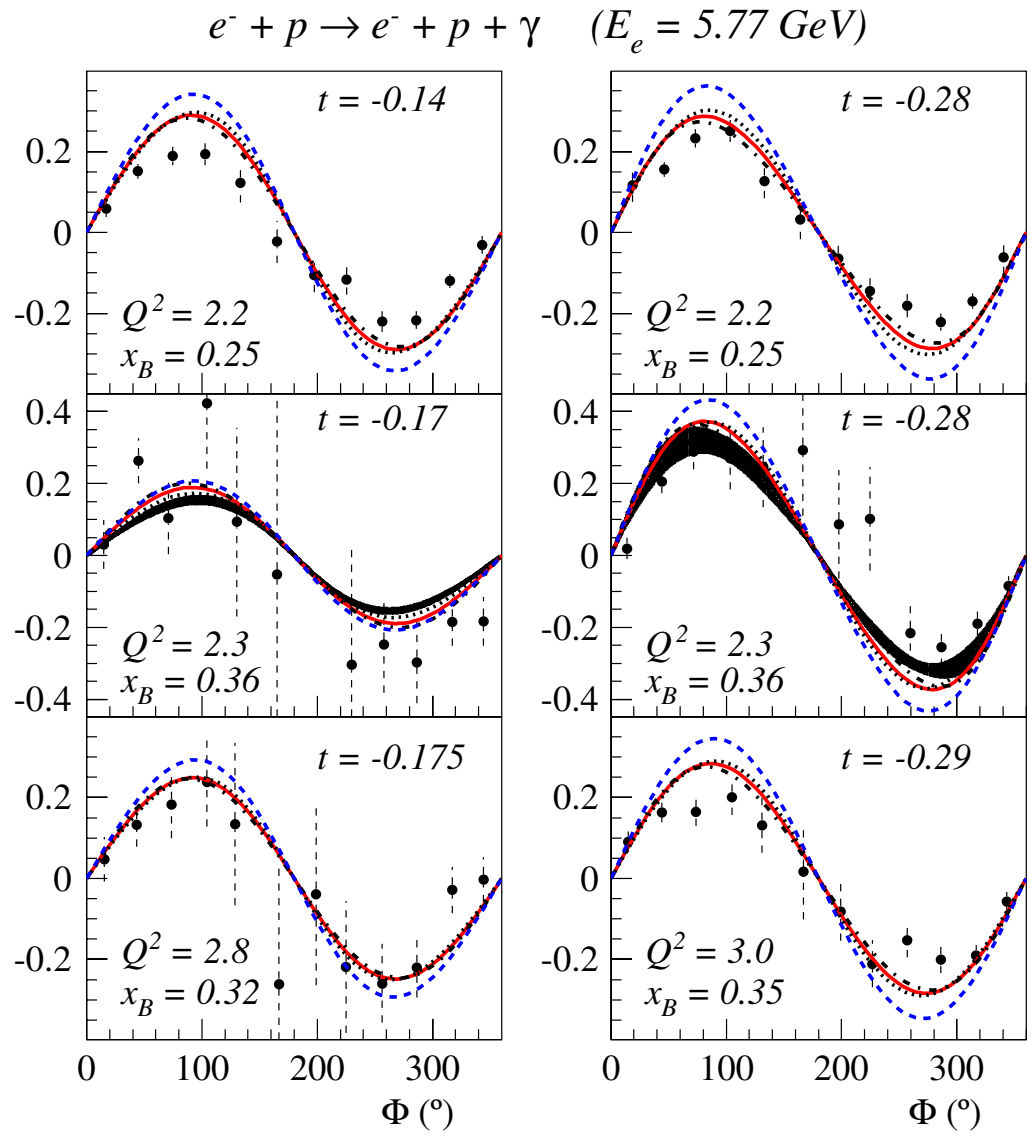

Figure 39. Azimuthal dependence of the BSA in DVCS in several kinematic bins. The black bands in the two middle panels are JLAB data from [226], while the data points are JLAB data from [229]. The curves are explained in the text. The figure is taken from [213].

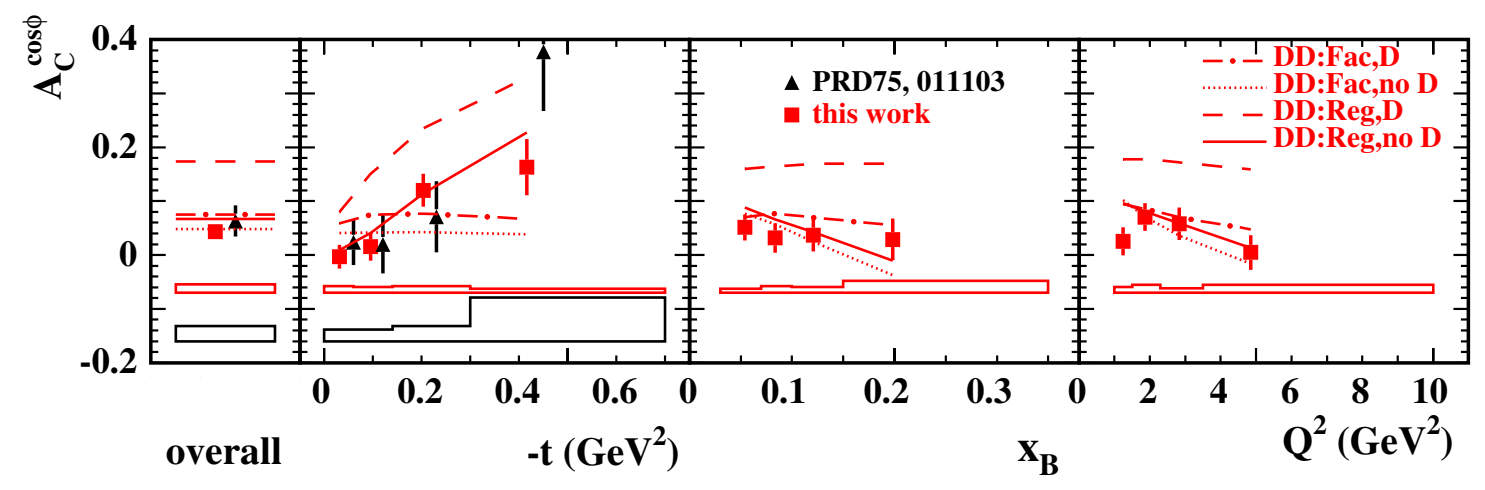

Figure 40. BCA measured on the proton by HERMEs: azimuthal amplitude describing the dependence of the interference term on the beam charge $\left(A_{\mathrm{C}}\right)$. The triangles (shifted right for visibility) represent previous results [232], while most recent data [233] are represented by squares. The error bars (bands) represent the statistical (systematic) uncertainties. The curves labelled 'DD' are calculations of variants of a DD GPD model $[125,198]$ using $b_{\mathrm{v}}=\infty$ and $b_{\mathrm{s}}=1$ as profile parameters for valence and sea quarks. This figure is extracted from figure 5 of [233].

components, 'normal' to and 'sideways' in the production plane [236], defined by the directions of the virtual and real photons. The DVCS cross section depends only on $\widehat{M}_{\mathrm{T}}$, the normal component of the twist-2 Compton amplitude (see (65)). This is the only amplitude that embodies a combination of CFFs in which the contributions of the quark GPDs $E^{f}$ are not kinematically suppressed relative to those of $H^{f}$. Hence DVCS measurements on a transversely polarized proton target appear to be crucial for the evaluation of $J_{u}$ and $J_{d}$ through the Ji relation (37). A complication inherent to the exploitation of this relation lies in the fact that both quark GPDs $H^{f}$ and $E^{f}$ need to be determined in the limit $t \rightarrow 0$, whereas at small $t$ the relevant asymmetry is suppressed by a factor of $\sqrt{t_{0}-t} / 2 m$ when extracting the GPDs $H^{f}$ and even by a factor of $\left(t_{0}-t\right) / 4 m^{2}$ when extracting the GPDs $E^{f}$ (which is apparent from a comparison of (63) and (65)). The extraction of model-dependent constraints on $J_{u}$ and $J_{d}$ from experimental data is discussed in section 4.8 .

From data taken with both beam charges and transverse target polarization $S_{\perp}$, the BCA $\mathcal{A}_{\mathrm{C}}(\phi)$ (see (93)) can 


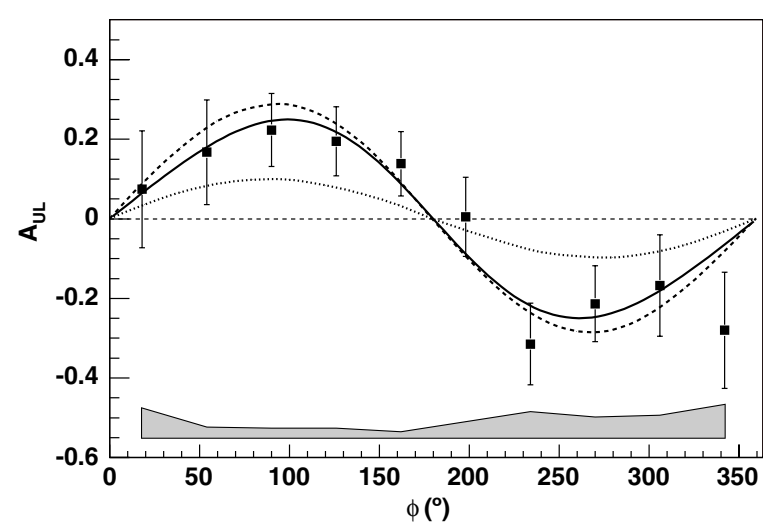

Figure 41. Azimuthal dependence of the longitudinal target-spin asymmetry, as measured at JLAB [234] with $5.7 \mathrm{GeV}$ electrons and a longitudinally polarized $\mathrm{NH}_{3}$ target, shown after subtraction of the $\pi^{0}$ background. Error bars represent the statistical uncertainties and the band at the bottom represents the systematic uncertainties. The curves are explained in the text. Reprinted with permission from [234]. Copyright (2006) by the American Physical Society.

be extracted simultaneously with the transverse target-spin asymmetries arising from the $\left|\tau_{\mathrm{DVCS}}\right|^{2}$ and interference terms (see (61)), respectively:

$$
\begin{aligned}
& \mathcal{A}_{\mathrm{UT}}^{\mathrm{DVCS}}\left(\phi, \phi_{S}\right) \equiv \frac{1}{S_{\perp}} \cdot\left[\mathrm{d} \sigma^{+}\left(\phi, \phi_{S}\right)-\mathrm{d} \sigma^{+}\left(\phi, \phi_{S}+\pi\right)\right. \\
& \left.\quad+\mathrm{d} \sigma^{-}\left(\phi, \phi_{S}\right)-\mathrm{d} \sigma^{-}\left(\phi, \phi_{S}+\pi\right)\right]\left[\mathrm{d} \sigma^{+}\left(\phi, \phi_{S}\right)\right. \\
& \left.+\mathrm{d} \sigma^{+}\left(\phi, \phi_{S}+\pi\right)+\mathrm{d} \sigma^{-}\left(\phi, \phi_{S}\right)+\mathrm{d} \sigma^{-}\left(\phi, \phi_{S}+\pi\right)\right]^{-1},
\end{aligned}
$$

$$
\begin{aligned}
& \mathcal{A}_{\mathrm{UT}}^{I}\left(\phi, \phi_{S}\right) \equiv \frac{1}{S_{\perp}} \cdot\left[\mathrm{d} \sigma^{+}\left(\phi, \phi_{S}\right)-\mathrm{d} \sigma^{+}\left(\phi, \phi_{S}+\pi\right)\right. \\
& \left.-\mathrm{d} \sigma^{-}\left(\phi, \phi_{S}\right)+\mathrm{d} \sigma^{-}\left(\phi, \phi_{S}+\pi\right)\right]\left[\mathrm{d} \sigma^{+}\left(\phi, \phi_{S}\right)\right. \\
& \left.+\mathrm{d} \sigma^{+}\left(\phi, \phi_{S}+\pi\right)+\mathrm{d} \sigma^{-}\left(\phi, \phi_{S}\right)+\mathrm{d} \sigma^{-}\left(\phi, \phi_{S}+\pi\right)\right]^{-1} .
\end{aligned}
$$

The azimuthal angle $\phi_{S}$ of the target polarization vector was explained in section 3.4. The subscripts on the $\mathcal{A}$ s represent the dependence on beam charge $(\mathrm{C})$ or transverse $(\mathrm{T})$ target polarization, with an unpolarized (U) beam, and the superscripts \pm stand for the lepton beam charge. All three asymmetries are expanded in terms of various harmonics in $\phi$ and $\phi_{S}$, which respective amplitudes are hereafter called '(effective) azimuthal (asymmetry) amplitudes'.

Only a few azimuthal amplitudes are expected to show substantial sensitivity to $J_{u}$ and $J_{d}$ [237]. At HERMES all harmonics of physical significance were measured with unpolarized beam and a transversely polarized proton target [233]. The kinematic dependences of two asymmetry amplitudes of particular interest are shown in figure 42 in comparison with calculations based on two different types of GPD models. The data are clearly able to discriminate among these GPD models. The model variants that agree best with the data are used in the next section to derive constraints from the data on the total angular momentum of $u$ quarks. Sensitivity exists, as can be seen from the top panel of the figure.

\subsection{Quark total angular momenta}

In the theoretical description of the DVCS process, experimental observables like cross section differences or asymmetries are related to various combinations of the CFFs $\mathcal{H}, \mathcal{E}, \widetilde{\mathcal{H}}, \widetilde{\mathcal{E}}$, which can be calculated from the respective GPDs $H^{f}, \widetilde{H}^{f}, E^{f}, \widetilde{E}^{f}$ (see section 4.5). Parametrizations of the spin-flip GPD $E^{f}$ embody explicitly or implicitly the quark total angular momenta $J_{u}$ and $J_{d}$ [125]. Hence modeldependent constraints on these two parameters can be derived by fitting them to experimental observables [237]. The DD model [125, 198] was thereby fitted to azimuthal amplitudes of beam-charge and transverse target-spin asymmetries measured on the proton at Hermes (see previous section). The resulting constraint [233] is shown in figure 43 as the sloped band, which in units of $\hbar$ can be represented as

$$
J_{u}+J_{d} / 2.8=0.49 \pm 0.17\left(\exp _{\mathrm{tot}}\right) .
$$

Combining DVCS measurements on deuteron and proton targets, the beam-spin difference for the neutron was determined at JLAB [238]. Also in this case sensitivity to the GPDs $E^{f}$ can be found, as for the neutron the prefactor $F_{1}(t)$ in $(63)$ is small but $F_{2}(t)$ is not. Similarly as described above, a model-dependent constraint on $\left(J_{u}, J_{d}\right)$ was derived by comparison with a calculation of the DD model. The slope of the resulting band differs from that of the band derived from proton data (see figure 43) due to the different quark contents of proton and neutron:

$$
J_{u}+J_{d} / 5.0=0.18 \pm 0.14\left(\exp _{\text {tot }}\right) .
$$

These results show that the existing data have the potential to provide quantitative information about the quark total angular momenta $J_{u}$ and $J_{d}$. However, as discussed in the previous sections, the DD model of $[125,198]$ cannot explain all existing DVCS data.

\section{The spin budget of the nucleon}

\subsection{Decomposing the nucleon spin}

While the total angular momentum of an isolated system is uniquely defined, ambiguities arise when decomposing the total angular momentum of an interacting multi-constituent system into contributions from various constituents. Moreover, in a gauge theory, switching the gauge may result in shuffling angular momentum between matter and gauge degrees of freedom. In the context of nucleon structure, this gives rise to subtleties in defining these quantities that are more fundamental than those subtleties associated with the choice of factorization scheme, mentioned in section 2.4.

In section 4.1, it was discussed how GPDs can be used to determine the quark total angular momentum contribution $J_{f}$ to the nucleon spin (see (37)) in a gauge invariant decomposition of the nucleon's spin $\frac{1}{2}$. The expectation value of the $\hat{z}$ component of the quark orbital angular momentum operator can then be evaluated indirectly as the difference,

$L_{q}=\sum_{f} L_{f}=\sum_{f}\left[J_{f}-\frac{1}{2}\left(\Delta q_{f}+\Delta \bar{q}_{f}\right)\right]=J_{q}-\frac{1}{2} \Delta \Sigma$, 


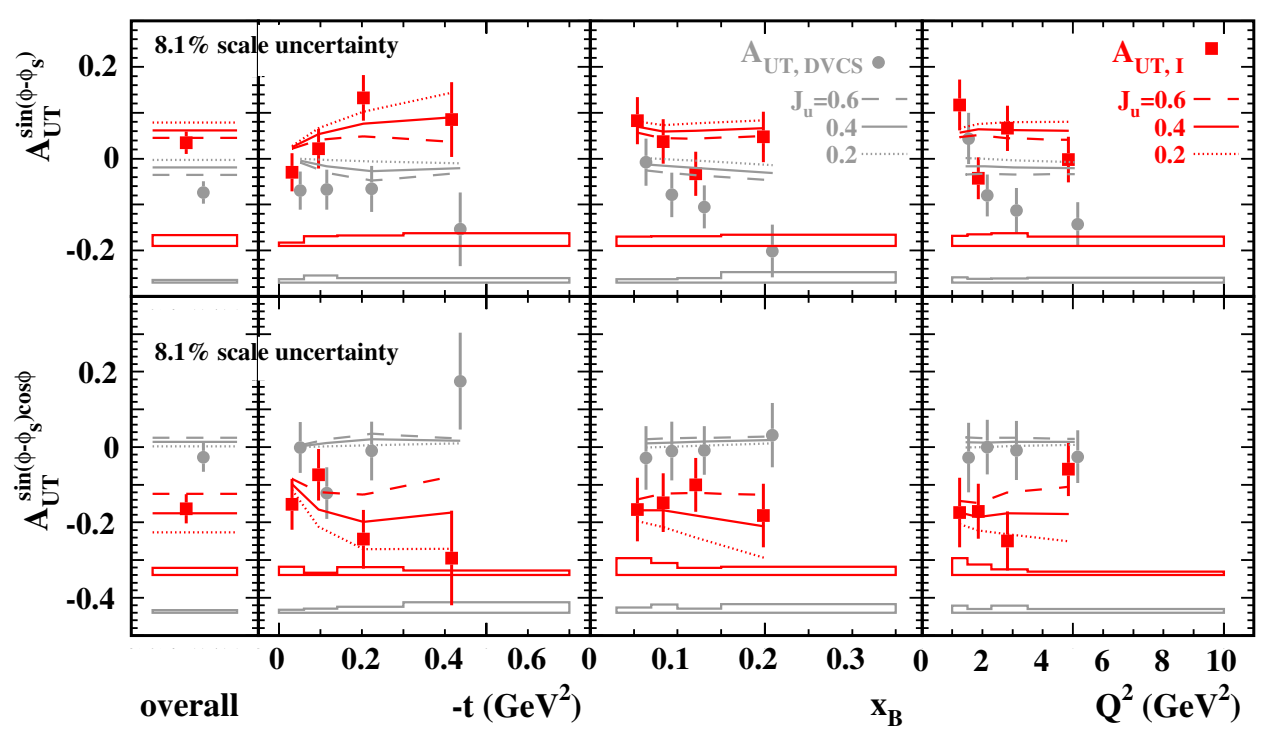

Figure 42. Asymmetry amplitudes describing the dependence of the squared DVCS amplitude (circles, $A_{\mathrm{UT}, \mathrm{DVCS}}$ ) and the interference term (squares, $A_{\mathrm{UT}, \mathrm{I}}$ ) on the transverse target polarization. The circles (squares) are shifted right (left) for visibility. The error bars represent the statistical uncertainties, while the top (bottom) bands denote the systematic uncertainties for $A_{\mathrm{UT}, \mathrm{I}}\left(A_{\mathrm{UT}, \mathrm{DVCS}}\right.$ ), excluding an $8.1 \%$ scale uncertainty from the target polarization measurement. The curves in the upper panel are calculations of the GPD model variant (Reg, no $D$ ) with three different values for the $u$-quark total angular momentum $J_{u}$ and fixed $d$-quark total angular momentum $J_{d}=0$ [237]. This figure is based on figure 6 of [233].

which is otherwise presently inaccessible experimentally. Similarly one can indirectly evaluate the expectation value of the operator representing the gluon total angular momentum as the difference between the nucleon's spin $\frac{1}{2}$ and $J_{q}$ :

$$
J_{\mathrm{G}}=\frac{1}{2}-J_{q} .
$$

One might consider going even further and use experimental results for $\Delta G$ to determine the gluon orbital angular momentum as the difference between $J_{\mathrm{G}}$ and $\Delta G$, providing what appears to be a complete understanding for the nucleon spin budget. However, the difference between these two operators has no fundamental connection to orbital angular momentum other than being the difference between $J_{\mathrm{G}}$ and $\Delta G$.

Equation (100) is a special case of Ji's decomposition [19]

$$
\vec{J}=\sum_{f}\left(\vec{S}_{f}+\vec{L}_{f}\right)+\vec{J}_{\mathrm{G}}
$$

of the expectation value of the angular momentum operator. Since this decomposition of the angular momentum density $M^{\mu \nu \lambda}=T^{\mu \nu} x^{\lambda}-T^{\mu \lambda} x^{\nu}$, where $T^{\mu \nu}=T^{\nu \mu}$ is the energymomentum tensor, in terms of manifestly gauge invariant local operators (see footnote 11) is based on the decomposition of the $M^{0 i j}$-component $(i, j \in\{x, y, z\})$, it is independent of the quantization axis. Here $\vec{S}_{f}$ is defined through the expectation value of the axial vector current $\bar{\psi}_{f} \vec{\gamma} \gamma_{5} \psi_{f}, \vec{L}_{f}$ through the expectation value of $\psi_{f}^{\dagger} \vec{r} \times(\vec{p}-g \vec{A}) \psi_{f}$, and $\vec{J}_{\mathrm{G}}$ through the expectation value of $\vec{r} \times \operatorname{Tr}(\vec{E} \times \vec{B})$, where $\vec{E}$ and $\vec{B}$ are the QCD colour electric and magnetic fields. A further decomposition of $\vec{J}_{\mathrm{G}}$ into spin and orbital components using manifestly gauge invariant local operators has not been found and may be impossible. In general, spin (or 'intrinsic' angular momentum) is identified with terms in the operator that do not depend explicitly on $\vec{r}$, in contradistinction to the orbital (or 'extrinsic') piece [241].

Being based on manifestly gauge invariant local operators, all three terms in (102) can be calculated in lattice gauge theory. As discussed in previous sections, the expectation values $\vec{S}_{f}$ and $\vec{J}_{f}$ can be accessed experimentally, and $\vec{J}_{\mathrm{G}}$ can, at least in principle, be accessed in exclusive deeply virtual heavy meson production. Beyond leading order in $\alpha_{s}$, all three terms in (102) depend on $Q^{2}$, but the $Q^{2}$ dependence cancels in their sum.

The main disadvantage of decomposition (102) is that for no choice of the quantization axis is there a partonic interpretation as a difference between number densities for the quark orbital angular momentum $\vec{L}_{f}$ or the gluon total angular momentum $\vec{J}_{\mathrm{G}}$. Only the quark intrinsic angular momentum $\vec{S}_{f}$ has such an interpretation (for the $\hat{z}$ quantization axis). This is because the operator definitions for $\vec{L}_{f}$ and $\vec{J}_{\mathrm{G}}$ contain interactions (e.g. $\vec{L}_{f}$ contains the gluon field through the gauge-covariant derivative $\vec{D}=\vec{\partial}-\mathrm{i} g \vec{A}$ ). With quarks and gluons thereby inextricably intertwined, it is impossible to unambiguously identify these terms with quark orbital angular momentum and gluon angular momentum, respectively [242].

An alternative 'light-cone decomposition' was proposed by Jaffe and Manohar [243] (see also [244, 245]). While the Ji decomposition is based on the $M^{0 i j}$ component of the angular momentum density, the Jaffe-Manohar decomposition is based on the $M^{+x y}$ component and applies only to the $z$ component of $\vec{J}$. It can be written as

$$
\frac{1}{2}=J_{z}=\frac{1}{2} \Delta \Sigma+\Delta G+\mathcal{L} .
$$

The quantity $\Delta \Sigma$ appears also in (100), the special case of (102) in the helicity basis. The quark-intrinsic piece is the same in both components: the operator that measures this piece in 


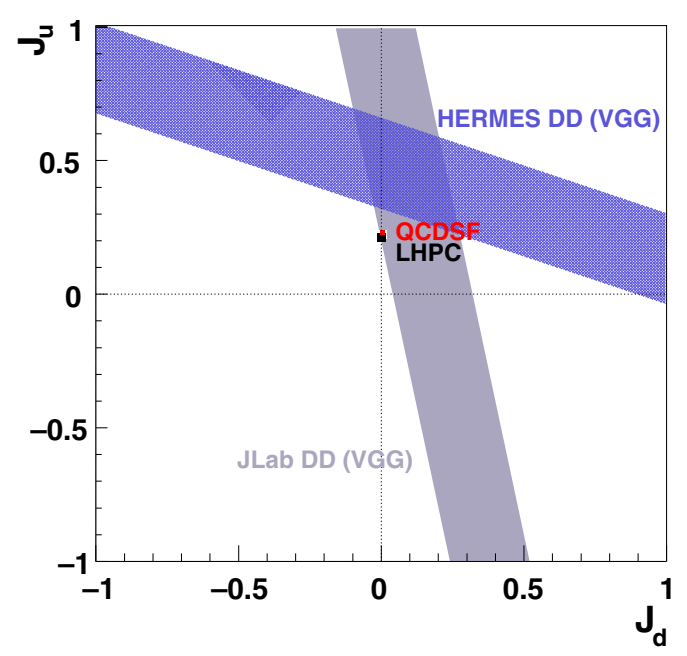

Figure 43. Model-dependent constraints on $u$-quark total angular momentum $J_{u}$ versus $d$-quark total angular momentum $J_{d}$, obtained by comparing DVCS experimental results and theoretical calculations using the DD GPD model of $[125,198]$. The constraints based on the HERMES data [233] for the azimuthal amplitudes $A_{\mathrm{UT}, \mathrm{I}}^{\sin \left(\phi-\phi_{S}\right) \cos \phi}$ and $A_{\mathrm{UT,I}}^{\sin \left(\phi-\phi_{S}\right)}$ are labelled HeRMES DD (VGG). Those based on the JLAB neutron cross section data [238] are labelled JLAB DD (VGG). Also shown as small (overlapping) rectangles are results from lattice gauge theory by the QCDSF [239] and LHPC [165] collaborations, as well as a result for only the valence-quark contribution (DFJK) based on zero-skewness GPDs extracted from nuclear form factor data [193,240]. The sizes of the small rectangles represent the statistical uncertainties of the lattice results and the parameter range for which a good DFJK fit to the nucleon form factor data was achieved. Theoretical uncertainties are unavailable. The figure is taken from [233].

$M^{+12}$ is $\epsilon^{+12-} \bar{\psi} \gamma_{-} \gamma_{5} \psi$ and the operator that measures it in $M^{012}$ is $\epsilon^{0123} \bar{\psi} \gamma_{3} \gamma_{5} \psi$, i.e. both operators are components of the axial vector current and therefore their expectation values are proportional to the axial charge, so that $\Delta \Sigma$ appears in both decompositions. This is not true for any other term in the decompositions! One can understand this point by analogy with the energy-momentum tensor $T^{\mu \nu}$ [242]: the energy of a single particle state can be expressed in terms of the matrix element of $T^{00}$ and decomposed into a quark and gluon piece. The 'momentum' in the infinite-momentum frame corresponds to the matrix element of $T^{++}$and can also be decomposed into quark and gluon pieces. The quark and gluon pieces of these two objects are unrelated.

In decomposition (103), each term has a partonic interpretation. The gluon spin contribution $\Delta G$ appears explicitly. It is experimentally accessible (see section 2.8) and can be defined as the expectation value of a (non-local) manifestly gauge invariant operator. In light-cone gauge, this operator collapses to a local operator (and its expectation value has a partonic interpretation). No direct experimental access to the parton orbital angular momentum $\mathcal{L}$ has been identified. Its value can be obtained only by subtracting the quark and gluon spin contributions from the nucleon spin. Both $\Delta G$ and $\mathcal{L}$ can be defined through matrix elements of local operators only in light-cone gauge $A^{+}=0$. Explicit definitions for the operators appearing in both decompositions can be found in [242]. Since neither one can be represented as the matrix element of a manifestly gauge invariant local operator, they cannot be analytically continued to Euclidean space and are thus inaccessible for lattice QCD.

One can decompose $\mathcal{L}$ further into contributions from gluons and each quark flavour:

$$
\mathcal{L}=\sum_{f} \mathcal{L}_{f}+\mathcal{L}_{\mathrm{G}}
$$

where, for example, the contribution $\mathcal{L}_{f}$ from quarks with flavour $f$ can be obtained from the expectation value of $\psi_{f+}^{\dagger}\left(x p_{y}-y p_{x}\right) \psi_{f+}$ (with $\psi_{f+}$ being the dynamical or 'good' component of the quark field operator $\psi_{f}$ in that framework) for a nucleon polarized along the $\hat{z}$-direction. Each of the terms in (104) has a partonic interpretation. Even a decomposition with respect to the momentum fraction $x$ is possible, but for none of these terms has experimental access been identified nor are they accessible in lattice QCD, since their manifestly gauge invariant forms are non-local in Minkowski space [246]. Nevertheless, their expectation values can be easily related to the (light-cone) wave functions of hadrons, which play an important role in phenomenology [247].

Summarizing the above dichotomy, the two decompositions described above have only $\Delta \Sigma$ in common, which is also manifestly gauge invariant and experimentally accessible. The experimentally accessible quantities $J_{f}$ (and, by subtraction, $L_{f}$ and $J_{\mathrm{G}}$ ) appear in one decomposition, while $\Delta G$ (and, by subtraction, $\mathcal{L}$ ) appear in the other. Combining terms from these incommensurate decompositions would be a sterile exercise. For example, after determining $J_{\mathrm{G}}=\frac{1}{2}-\sum_{f} J_{f}$ by evaluating the right-hand side using the $\mathrm{Ji}$ relation for $J_{f}$, it would be fruitless to 'determine' $L_{\mathrm{G}}$ by subtracting $\Delta G$ from $J_{\mathrm{G}}$ (or equivalently by subtracting $L_{q}$ from $\mathcal{L}$ ). In fact, the last step would yield a term that is neither directly experimentally accessible nor could it be represented by an operator other than that defined as the difference of the two operators. Defining gluon orbital angular momentum through such a subtraction procedure has no predictive power (not even in principle).

Note that an analogous dichotomy (multichotomy) appears when one considers the motion of a charged particle in an electromagnetic field. While the canonical momentum $\boldsymbol{p} \equiv-\mathrm{i} \nabla$ has a simple interpretation in terms of only the charged particle's wave function, it is not gauge invariant. Evaluation of the gauge invariant combination $\boldsymbol{p}-e \boldsymbol{A}$ requires in addition knowledge about the vector potential. This combination is directly accessible experimentally, e.g., on a macroscopic scale by studying the trajectory of the particle in a magnetic field. Similarly, while the canonical orbital angular momentum $\boldsymbol{r} \times \boldsymbol{p} \equiv-\mathrm{i} \boldsymbol{r} \times \nabla$ has a simple interpretation as a property of the charged particle's wave function alone, it is unfortunately not gauge invariant. On the other hand, the gauge invariant combination $\boldsymbol{r} \times(\boldsymbol{p}-\boldsymbol{e A})$ contains the vector potential $\boldsymbol{A}$ and its interpretation is therefore more subtle. Furthermore, only the gauge non-invariant orbital angular momentum $\boldsymbol{r} \times \boldsymbol{p}$ satisfies the familiar angular momentum commutation relations $\left[L_{i}, L_{j}\right]=\mathrm{i} \varepsilon^{i j k} L_{k}$. In the end, both 
definitions of the angular momentum of the charged particle have their merit, and depending on the context either may be preferable.

Analogously, in the decomposition of the nucleon spin, it also turns out that both schemes have their respective merits. In Ji's decomposition both $J_{q}$ and $J_{\mathrm{G}}$ are manifestly gauge invariant and calculable in lattice gauge theory (see section 4.4). Furthermore, using (37) $J_{f}$ can be measured, which allows determination of $L_{f}=J_{f}-\frac{1}{2}\left(\Delta q_{f}+\Delta \bar{q}_{f}\right)$. However, since it is even more difficult to relate $J_{\mathrm{G}}$ to measurable quantities (e.g. using (38)) than is the case for $J_{f}$, the relation can currently not be 'tested', while it can nevertheless be used to determine $J_{\mathrm{G}}=\frac{1}{2}-J_{q}$, which is interpreted as gluon total angular momentum in the equal time quantized framework only.

Besides a partonic interpretation of all terms, the JaffeManohar decomposition (103) has the advantage that it contains the experimentally accessible quantity $\Delta G$ (see section 2.8). Unfortunately, also here no experimental observable has been found to relate to $\mathcal{L}_{f}$ or $\mathcal{L}_{\mathrm{G}}$ even in principle, so the light-cone based spin decomposition cannot be tested either, but one can still use the sum rule to determine the net parton orbital angular momentum $\mathcal{L}$. Another disadvantage of the light-cone based decomposition (103) is that because the operators used to define its terms (except $\Delta \Sigma$ ) are not manifestly gauge invariant, its terms cannot be calculated in lattice gauge theory. Nevertheless, although a simple (i.e. local) operator expression for $\Delta G$ exists only in light-cone gauge, it can be defined through a manifestly gauge invariant operator. In all other gauges, $\Delta G$ is described by matrix elements of a non-local operator rendering its calculation or interpretation exceedingly difficult. $\Delta G$ has a physical interpretation as the gluon intrinsic angular momentum in the light-cone framework only.

The two decompositions of the nucleon spin discussed here are not the only possibilities. For example, another alternative has been proposed in [248, 249]. However, it neither has a partonic interpretation nor does its orbital angular momentum seem to be experimentally accessible and we will not discuss this decomposition here.

An analogy may illustrate the dangers in mixing components from different decompositions: suppose hypothetically that DIS data on deuterium were available only at $Q^{2}=100 \mathrm{GeV}^{2}$ and proton data were available only at $Q^{2}=10 \mathrm{GeV}^{2}$. The normal procedure for extracting neutron data would be to evolve to a common $Q^{2}$ value first. However, again hypothetically, suppose there existed no evolution equations. One may then ask oneself whether it would make sense (without first evolving the results from one $Q^{2}$ to the other value of $Q^{2}$ ) to subtract the distribution of $u$ quarks in the proton at $Q^{2}=10 \mathrm{GeV}^{2}$ from that in the deuteron at $Q^{2}=100 \mathrm{GeV}^{2}$ and use the result to define the distribution of $u$ quarks in the neutron. The main weakness of this analogy is of course that this definition is falsifiable because experiments may be performed at different values of $Q^{2}$ or QCD evolution may be discovered.

\subsection{Implications for quark angular momenta}

5.2.1. Results using the Ji relation. Due to the difficulties in extracting GPDs from DVCS data, no model-independent experimental constraints on the Ji relation yet exist. Latticebased constraints on $J_{f}$ have been discussed in section 4.4 and indicate that $L_{u}+L_{d}$ is consistent with zero, while $L_{u}-L_{d} \approx-0.4 \pm 0.05$. In that subsection it was already emphasized that this result is counterintuitive, where the intuition derives from comparison with constituent quark models and phenomenology for single-spin asymmetries. Given the complementary and incommensurate definitions of quark orbital angular momentum, as discussed in the previous section, the question arises whether the orbital angular momentum in these models should be identified with $L_{f}$ in (102) or with $\mathcal{L}_{f}$ in $(103,104)$. If they were to be identified with $L_{f}$, there would be additional, and presently unknown, corrections added to account for the difference before the orbital angular momentum in the models could be compared with the lattice QCD results.

5.2.2. Results in the light-cone decomposition. The existing information on $\Delta G$ from global fits of experimental data suggests that its first moment is small [65]. Neglecting 'theoretical' and model uncertainties such as those associated with the choice of functional forms used to represent PDFs in the fits while interpreting table 2 leads to $\Delta G \simeq-0.1 \pm$ 0.1 (expt.) for $\Delta \chi^{2}=1$. Using the most precise result for $\Delta \Sigma=0.33 \pm 0.03$ (tot.) [47] the net parton orbital angular momentum is obtained from (103) as

$$
\mathcal{L}=\sum_{f} \mathcal{L}_{f}+\mathcal{L}_{\mathrm{G}} \simeq 0.43 \pm 0.1,
$$

which would be more than twice the intrinsic contribution from quarks. It remains open how gluons and quarks share their orbital contributions.

\section{Conclusions and outlook}

One measure of the vitality of a field is the rapidity with which its conceptual framework and focus evolve. By this measure, the study of the internal spin structure of the proton is indeed vital. Its explosive birth in the late 1980s was triggered by the development of experimental technologies for DIS of polarized high-energy leptons on targets containing polarized protons. The pivotal EMC measurement $[17,18]$ suggested that the helicities of the quarks combine to make a net contribution to the helicity of the proton that is small compared with naive expectations. This finding inspired a flood of theoretical papers and several major new experimental efforts at three laboratories, which confirmed the original finding with much higher precision, and extended the measurements to the neutron target to help distinguish the contributions of the various quark flavours. While $u$ quarks were found to be strongly polarized in the same direction as the parent proton, both $d$ quarks and even $s$ quarks were found to be negatively polarized. These measurements of inclusive DIS asymmetries were extended to semi-inclusive measurements where the type 
of hadron produced in the fragmentation of the struck quark tends to 'tag' the flavour of that quark. The resulting data indicate that the helicity densities of sea quarks are small in the measured $x$ range of $0.02<x<0.4$, but still consistent with fits to inclusive data yielding a negative first moment for the strange sea, which receives a significant negative contribution from $x<0.02$.

Attention soon focused on a possibly substantial role of the helicity of the gluons, which is experimentally difficult to probe in DIS. While some of the existing new experiments struggled to extract constraints on the gluon polarization, yet more new efforts were launched that were designed specifically to target this problem. At the time of writing, these are beginning to bear fruit, suggesting that the fractional gluon helicity contribution is small, and together with the quark helicities, does not exhaust the 'spin budget' of the proton. This leaves the question of the contribution of parton orbital angular momentum. In the light-cone framework for defining this quantity with a partonic interpretation in the axial gauge, one can estimate it as the difference $\mathcal{L}=\frac{1}{2}-\frac{1}{2} \Delta \Sigma-\Delta G$. Although $\Delta \Sigma$ is precisely known from measurements of $g_{1}^{d}$, the first moment $\Delta G$ is still so poorly known that there is still little direct experimental constraint on the contribution of parton orbital angular momentum to the nucleon spin.

In the 1990s, dramatic theoretical progress reshaped the field, identifying new experimental signals for nucleon spin structure. One key issue has been quark transversity, which differs from helicity because of the relativistic internal motion of quarks. It was realized that single-spin asymmetries in semiinclusive scattering offered access to this elusive property, through a dependence of the fragmentation process on the transverse polarization of the struck quark - the Collins effect. While initial experimental evidence for this phenomenon was ambiguous, eventually SIDIS measurements with transverse target polarization in combination with studies of the jet structure of hadrons from $\mathrm{e}^{+} \mathrm{e}^{-}$collisions provided a data set with a clear interpretation, leading to the first extraction of transversity distributions. As expected, they are found to share the features of the helicity distributions in that they are positive for $u$ quarks, and negative and considerably smaller for $d$ quarks. Their magnitudes are found to be about half of the Soffer positivity bounds, which are expressed in terms of the unpolarized and helicity densities.

One of the ambiguities that complicated the interpretation of early measurements of single-spin asymmetries was a possible contribution by the Sivers effect, which arises from a 'naively T-odd' correlation between the nucleon spin and the intrinsic transverse momentum of quarks. This developed into a fascinating topic of its own when it was realized that such distributions raise new questions about key theorems for factorization of hard and soft dynamics of processes, and the importance of ISIs or FSIs in the understanding of parton distributions that depend on intrinsic transverse momentum. The above-mentioned SIDIS measurements with transverse target polarization also provided clear evidence of the Sivers effect. Future high-luminosity measurements of the DY process with transversely polarized protons are now anticipated as a test of the new prediction that the Sivers effect will appear there with the opposite sign.
The pursuit of parton orbital angular momentum also led to dramatic theoretical progress in the late 1990s. It was realized that experimental access to this quantity might be provided by hard exclusive processes that involve hard interactions, yet leave the target nucleon intact. Most prominent among these processes is DVCS, the production of a single energetic photon, because of its fruitful interference with the well-understood $\mathrm{BH}$ process, providing access to the phase of the DVCS amplitude. Such exclusive processes are now interpreted within the framework of GPDs that depend on three kinematic variables and subsume both elastic form factors as moments and ordinary parton distributions as special cases. The excitement centred around the finding that a second moment of certain GPDs provides the total angular momentum of partons, including orbital angular momentum. Although existing DIS experimental facilities were not designed to measure exclusive processes, they managed to make some pioneering measurements, which have been compared with calculations based on the few available GPD parametrizations. While the basic features of the data are described, none of the existing GPD models are able to simultaneously explain all available DVCS data, including cross sections and their differences or asymmetries with respect to beam charge and helicity, and target polarization. Nevertheless, the data for transverse target polarization were shown to be sensitive to quark total angular momentum, of which the contribution from quark intrinsic spin has already been determined as mentioned above. Hence the development of more successful parametrizations can be expected to thereby result in constraints on quark orbital angular momentum. Promising efforts already underway based on 'conformal moments' offer a practical means of fitting DVCS data with parametrizations at NLO in $\alpha_{s}$. Some success with this approach has been achieved with DVCS cross sections at collider energies, and work continues to extend this to charge and polarization dependences at fixed-target energies.

A decade ago, progress in the understanding of non-perturbative QCD was made mainly by comparing experimental data with models that were constructed to approximately embody some important features of QCD. Since then, rapid progress has been made in improving the scope and precision of numerical simulations of QCD on a Euclidean lattice. Improved algorithms and techniques together with rapidly increasing computational power have combined to bring this field to the point that results for a large variety of observables can now be taken seriously as implications of QCD. An example is the spin budget of the proton, where it is computed that the only substantial contribution by quarks is from the $u$ flavour, in which the positive contribution from quark intrinsic spin dominates the negative contribution from orbital angular momentum. While several types of important observables will remain inacessible to this approach for the forseeable future (such as the gluonic contribution to the spin budget), others that are calculable are so difficult to measure experimentally that lattice calculations will be the only source of information about them for a considerable time. Examples here include chiral-odd GPDs. Hence calculations on the lattice play a role that is highly complementary to both experiment and model building. 
The existing experimental facilities for polarized DIS have provided a wealth of new data, much of it unanticipated when those experiments were conceived. For example, they have provided high quality data for inclusive measurements of double-spin asymmetries, determining the spin structure function $g_{1}$ of the proton and neutron over the kinematic range of fixed-target experiments. However, other important avenues to nucleon spin structure such as semi-inclusive DIS and exclusive processes have been exploited with only enough precision to inspire and guide rapid theoretical progress, but not to provide detailed information. Also, the experience from the Hera e-p collider taught us that the vastly wider kinematic range available at such a collider can lead to unanticipated insights, in part because of the much improved access to the region of small $x$. One clear motivation for planning for a high-luminosity e-p collider with polarized beams is to obtain data for $g_{1}$ over a wide range in hard scale $Q^{2}$ to allow the precise inference of gluon polarization from the $Q^{2}$ evolution of $g_{1}$, in analogy to the success in extracting the unpolarized gluon density from Hera data. The rapidly expanding horizons of the field in the last decade have provided other compelling motivations. Only with high luminosity free of target dilution by unpolarized components and a broad kinematic range can precise information be produced in the study of transversity, transverse-momentumdependent parton distributions and quark orbital momentum through the constraint of GPDs. The clear need for a new accelerator facility to make this all possible has inspired specific proposals and design studies [250]. Luminosities of order $10^{33}$ nucleons $\left(\mathrm{cm}^{2} \mathrm{~s}^{-1}\right)$ are considered possible in the energy range $\sqrt{s}=15-100 \mathrm{GeV}$. The continuing vitality of this exciting field depends on the determined pursuit of this goal. While the community works towards this new facility, the present rate of rapid conceptual progress in the field will depend on a continuing flow of new data from Compass [89], the only remaining high-energy lepton DIS facility, as well as from Jefferson Laboratory, especially after it achieves its $12 \mathrm{GeV}$ upgrade [251].

\section{Acknowledgments}

The authors would like to warmly thank Markus Diehl, Klaus Rith and Werner Vogelsang for reading and providing a critique of a draft of this paper. The authors appreciate their helpful advice and comments, as well as those of Bob Jaffe and Christian Weiss. The authors are thankful to Eduard Avetisyan and Michael Engelhardt for technical help, and to Ulrike Elschenbroich for figures 2 and 3. MB was supported by the DOE under grant numbers DE-FG03-95ER40965 and DE-AC05-06OR23177 (under which Jefferson Science Associates, LLC, operates Jefferson Lab).

\section{References}

[1] Hofstadter R 1956 Rev. Mod. Phys. 28 214-54

[2] Bawin M and Coon S A 2001 Nucl. Phys. A 689 475-7 (arXiv:nucl-th/0101005)

[3] Gell-Mann M 1964 Phys. Lett. 8 214-5

[4] Zweig G 1964 CERN Report 8419/TH. 412
[5] Feynman R P 1969 Phys. Rev. Lett. 23 1415-7

[6] Bjorken J D 1969 Phys. Rev. 179 1547-53

[7] Gross D J and Wilczek F 1973 Phys. Rev. Lett. $301343-6$

[8] Politzer H D 1973 Phys. Rev. Lett. 30 1346-9

[9] Mueller A H 2001 arXiv:hep-ph/0111244

[10] Gribov V N and Lipatov L N 1972 Sov. J. Nucl. Phys. 15 438-50

[11] Dokshitzer Y L 1977 Sov. Phys.-JETP 46 641-53

[12] Altarelli G and Parisi G 1977 Nucl. Phys. B 126298

[13] Mertig R and van Neerven W L 1996 Z. Phys. C 70 637-54 (arXiv:hep-ph/9506451)

[14] Vogelsang W 1996 Phys. Rev. D 54 2023-9 (arXiv:hep-ph/9512218)

[15] Vogelsang W 1996 Nucl. Phys. B 475 47-72 (arXiv:hep-ph/9603366)

[16] Vogt A, Moch S, Rogal M and Vermaseren J A M 2008 Nucl. Phys. Proc. Suppl. 183 155-61 (arXiv:0807.1238 [hep-ph])

[17] Ashman J et al (EMC) 1988 Phys. Lett. B 206364

[18] Ashman J et al (EMC) 1989 Nucl. Phys. B 3281

[19] Ji X 1997 Phys. Rev. Lett. 78 610-13 (arXiv:hep-ph/9603249)

[20] Burkardt M 2000 Phys. Rev. D 62071503

Burkardt M 2002 Phys. Rev. D 66119903 (erratum) (arXiv:hep-ph/0005108)

[21] Diehl M 2002 Eur. Phys. J. C 25 223-32

Diehl M 2003 Eur. Phys. J. C 31277 (erratum) (arXiv:hep-ph/0205208)

[22] Belitsky A V, Ji X and Yuan F 2004 Phys. Rev. D 69074014 (arXiv:hep-ph/0307383)

[23] Ralston J P and Pire B 2002 Phys. Rev. D 66111501 (arXiv:hep-ph/0110075)

[24] Bjorken J 1966 Phys. Rev. 148 1467-78

[25] Gell-Mann M 1962 Phys. Rev. 1251062

[26] Gell-Mann M 1964 Physics 163

[27] Feynman R P, Gell-Mann M and Zweig G 1964 Phys. Rev. Lett. 13 678-80

[28] Jaffe R L 2001 AIP Conf. Proc. 588 54-74 (arXiv:hep-ph/0102281)

[29] Alguard M et al 1976 Phys. Rev. Lett. 371261

[30] Anthony P et al (E142) 1993 Phys. Rev. Lett. 71 959-62

[31] Abe K et al (E143) 1998 Phys. Rev. D 58112003 (arXiv:hep-ex/9802357)

[32] Abe K et al (E143) 1996 Phys. Rev. Lett. 76 587-91 (arXiv:hep-ex/0204028)

[33] Anthony P L et al (E155) 1999 Phys. Lett. B 463 339-45 (arXiv:hep-ex/9904002)

[34] Anthony P L et al (E155) 2000 Phys. Lett. B 493 19-28 (arXiv:hep-ph/0007248)

[35] Anthony P L et al (E155) 2003 Phys. Lett. B 553 18-24

[36] Adeva B et al (SMC) 1994 Phys. Lett. B 329 399-406 (arXiv:hep-ph/9404270)

[37] Adams D et al (SMC) 1995 Phys. Lett. B 357248

[38] Adeva B et al (SMC) 1997 Phys. Rev. D 56 5330-58 (arXiv:hep-ex/9702005)

[39] Adams D et al (SMC) 1997 Phys. Lett. B 396 338-48

[40] Adeva B et al (SMC) 1998 Phys. Rev. D 58112001

[41] Adeva B et al (SMC) 1999 Phys. Rev. D 60072004 Adeva B et al (SMC) 2000 Phys. Rev. D 62079902 (erratum)

[42] Adeva B et al (SMC) 2000 Phys. Rev. D 62079902

[43] Adeva B et al (SMC) 1998 Phys. Lett. B 420180

[44] Sokolov A and Ternov I 1964 Sov. Phys.-Dok. 81203

[45] Ackerstaff K et al (HERMES) 1997 Phys. Lett. B 404 383-9 (arXiv:hep-ex/9703005)

[46] Airapetian A et al (HERMES) 1998 Phys. Lett. B 442 484-92 (arXiv:hep-ex/0007248)

[47] Airapetian A et al (HERMES) 2007 Phys. Rev. D 75012007 (arXiv:hep-ex/0609039)

[48] Ageev E et al (COMPASS) 2005 Phys. Lett. B 612 154-64 (arXiv:hep-ex/0501073) 
[49] Zheng X et al (JLAB Hall A) 2004 Phys. Rev. C 70065207 (arXiv:nucl-ex/0405006)

[50] Anthony P et al (E142) 1996 Phys. Rev. D 54 6620-50 (arXiv:hep-ph/9610007)

[51] Abe K et al (E154) 1997 Phys. Rev. Lett. 79 26-30 (arXiv:hep-ex/9705012)

[52] Bardeen W 1978 Phys. Rev. D 183998

[53] Leader E, Sidorov A V and Stamenov D B 2007 Phys. Rev. D 75074027 (arXiv:hep-ph/0612360)

[54] Glück M et al 2001 Phys. Rev. D 63094005 (arXiv:hep-ph/0011215)

[55] Blümlein J and Böttcher H 2002 Nucl. Phys. B 636225 (arXiv:hep-ph/0203155)

[56] De Florian D, Navarro G and Sassot R 2005 Phys. Rev. D 71094018 (arXiv:hep-ph/0504155)

[57] Hirai M, Kumano S and Saito N (AAC) 2006 Phys. Rev. D 74014015 (arXiv:hep-ph/0603213)

[58] Adeva B et al (SMC) 1998 Phys. Rev. D 58112002

[59] Hirai M, Kumano S, Nagai T H and Sudoh K 2007 Phys. Rev. D 75094009 (arXiv:hep-ph/0702250)

[60] Albino S, Kniehl B A and Kramer G 2008 Nucl. Phys. B 803 42-104 (arXiv:0803.2768 [hep-ph])

[61] de Florian D, Sassot R and Stratmann M 2007 Phys. Rev. D 76074033 (arXiv:0707.1506 [hep-ph])

[62] Hillenbrand A 2005 Measurement and simulation of the fragmentation process at HERMES PhD Thesis University Erlangen-Nuremberg

[63] Airapetian A et al (HERMES) 2005 Phys. Rev. D 71012003 (arXiv:hep-ex/0407032)

[64] Alekseev M et al (COMPASS) 2008 Phys. Lett. B 660 458-65 (arXiv:0707.4077 [hep-ex])

[65] de Florian D, Sassot R, Stratmann M and Vogelsang W 2008 Phys. Rev. Lett. 101072001 (arXiv:0804.0422 [hep-ph])

de Florian D, Sassot R, Stratmann M and Vogelsang W 2009 arXiv:0904.3821 [hep-th]

[66] Navarro G A and Sassot R 2006 Phys. Rev. D 74011502 (arXiv:hep-ph/0605266)

[67] Kretzer S 2000 Phys. Rev. D 62054001 (arXiv:hep-ph/0003177)

[68] Towell R S et al (FNAL E866/NuSea) 2001 Phys. Rev. D 64052002 (arXiv:hep-ex/0103030)

[69] Dressler B, Goeke K, Polyakov M V and Weiss C 2000 Eur. Phys. J. C 14 147-57 (arXiv:hep-ph/9909541)

[70] Cao F G and Signal A I 2003 Phys. Rev. D 68074002 (arXiv:hep-ph/0306033)

[71] Eidelman S et al (Particle Data Group) 2004 Phys. Lett. B 5921

[72] Alexakhin V Y et al (COMPASS) 2007 Phys. Lett. B 647 8-17 (arXiv:hep-ex/0609038)

[73] Sjostrand Tet al 2001 Comput. Phys. Commun. 135 238-59 (arXiv:hep-ph/0010017)

[74] Liebing P 2004 Can the gluon polarization in the nucleon be extracted from HERMES on single high- $p_{t}$ hadrons $P h D$ Thesis Humboldt university, Berlin

[75] Liebing P (HERMES) 2007 Proc. of 17th Int. Spin Physics Symp. ed K Imai et al (AIP Conf. Proc. 915) pp 331-4 (arXiv:0707.3617 [hep-ex])

[76] Alekseev M et al (COMPASS) 2008 arXiv:0802.3023 [hep-ex]

[77] Ageev E S et al (COMPASS) 2006 Phys. Lett. B 633 25-32 (arXiv:hep-ex/0511028)

[78] Stolarski M (COMPASS) 2010 doi:10.3360/dis08.209 (arXiv:0809.1803 [hep-ex])

[79] Airapetian A et al (HERMES) 2000 Phys. Rev. Lett. 84 2584-8 (arXiv:hep-ex/9907020)

[80] Adeva B et al (Spin Muon (SMC)) 2004 Phys. Rev. D 70012002 (arXiv:hep-ex/0402010)

[81] Lai H L et al (CTEQ) 2000 Eur. Phys. J. C 12 375-92 (arXiv:hep-ph/9903282)
[82] Jager B, Stratmann M and Vogelsang W 2005 Eur. Phys. J. C 44 533-43 (arXiv:hep-ph/0505157)

[83] Jaffe R L 1996 arXiv:hep-ph/9602236

[84] Ratcliffe P G 2005 Eur. Phys. J. C 41 319-25(arXiv:hep-ph/0412157)

[85] Soffer J 1995 Phys. Rev. Lett. 74 1292-4(arXiv:hep-ph/9409254)

[86] Bakker B L G, Leader E and Trueman T L 2004 Phys. Rev. D 70114001 (arXiv:hep-ph/0406139)

[87] Leader E 2008 arXiv:0806.4879 [hep-ph]

[88] Efremov A V, Goeke K and Schweitzer P 2004 Eur. Phys. J. C 35 207-10 (arXiv:hep-ph/0403124)

[89] COMPASS 2009 CERN-SPSC-2009-003, SPSC-I-238 COMPASS 2009 CERN-SPSC-2009-025, SPSC-M769

[90] Mulders P J and Tangerman R D 1996 Nucl. Phys. B 461 197-237 (arXiv:hep-ph/9510301)

[91] Bacchetta A et al 2007 J. High Energy Phys. JHEP02(2007)093 (arXiv:hep-ph/0611265)

[92] Bacchetta A, Boglione M, Henneman A and Mulders P J 2000 Phys. Rev. Lett. 85 712-715 (arXiv:hep-ph/9912490)

[93] Collins J C and Metz A 2004 Phys. Rev. Lett. 93252001 (arXiv:hep-ph/0408249)

[94] Le Yaouanc A, Oliver L, Pene O and Raynal J C 1973 Phys. Rev. D 8 2223-34

[95] Artru X, Czyzewski J and Yabuki H 1997 Z. Phys. C 73 527-34 (arXiv:hep-ph/9508239)

[96] Collins J C 1993 Nucl. Phys. B 396 161-82 (arXiv:hep-ph/9208213)

[97] Sivers D W 1990 Phys. Rev. D 4183

[98] Collins J C 2002 Phys. Lett. B 536 43-8 (arXiv:hep-ph/0204004)

[99] Burkardt M 2007 arXiv:0709.2966 [hep-ph]

[100] Burkardt M 2002 Phys. Rev. D 66114005 (arXxiv:hep-ph/0209179)

[101] Burkardt M 2004 Nucl. Phys. A 735 185-99 (arXiv:hep-ph/0302144)

[102] Brodsky S J, Hwang D S and Schmidt I 2002 Phys. Lett. B 530 99-107 (arXiv:hep-ph/0201296)

[103] Boer D and Mulders P J 1998 Phys. Rev. D 57 5780-6 (arXiv:hep-ph/9711485)

[104] Adams D L et al (FNAL-E704) 1991 Phys. Lett. B 264 462-6

[105] Adams J et al (STAR) 2004 Phys. Rev. Lett. 92171801 (arXiv:hep-ex/0310058)

[106] Nogach L (STAR) 2007 AIP Conf. Proc. 915 543-6 (arXiv:hep-ex/0612030)

[107] Airapetian A et al (HERMES) 2000 Phys. Rev. Lett. 84 4047-51 (arXiv:hep-ex/9910062)

[108] Diefenthaler M (HERMES) 2010 doi:10.3360/dis07.91 (arXiv:0706.2242 [hep-ex])

[109] Alekseev M et al (COMPASS) 2008 Phys. Lett. B 673 127-35 (arXiv:0802.2160 [hep-ex])

[110] Bacchetta A, D'Alesio U, M D and Miller C A 2004 Phys. Rev. D 70117504 (arXiv:hep-ph/0410050)

[111] Anselmino M et al 2010 doi:10.3360/dis08.224 (arXiv:0807.0173 [hep-ph])

[112] Abe K et al (Belle) 2006 Phys. Rev. Lett. 96232002 (arXiv:hep-ex/0507063)

[113] Anselmino M et al 2009 Eur. Phys. J. A 39 89-100 (arXiv:0805.2677 [hep-ph])

[114] Airapetian A et al (HERMES) 2005 Phys. Rev. Lett. 94012002 (arXiv:hep-ex/0408013)

[115] Alexakhin V Y et al (COMPASS) 2005 Phys. Rev. Lett. 94202002 (arXiv:hep-ex/0503002)

[116] Sjostrand T 1994 Comput. Phys. Commun. 82 74-90

[117] Wakamatsu M 2001 Phys. Lett. B 509 59-68(arXiv:hep-ph/0012331)

[118] Schweitzer P et al 2001 Phys. Rev. D 64034013 (arXiv:hep-ph/0101300) 
[119] Pasquini B, Pincetti M and Boffi S 2005 Phys. Rev. D 72094029 (arXiv:hep-ph/0510376)

[120] Levorato S (COMPASS) 2008 arXiv:0808.0086 [hep-ex]

[121] de Florian D, Sassot R and Stratmann M 2007 Phys. Rev. D 75114010 (arXiv:hep-ph/0703242)

[122] Collins J C and Freund A 1999 Phys. Rev. D 59074009 (arXiv:hep-ph/9801262)

[123] Ji X 1997 Phys. Rev. D 55 7114-25 (arXiv:hep-ph/9609381)

[124] Diehl M, Feldmann T and Kroll P 2008 Phys. Rev. D 77033006 (arXiv:0711.4304 [hep-ph])

[125] Goeke K, Polyakov M V and Vanderhaeghen M 2001 Prog. Part. Nucl. Phys. 47 401-515 (arXiv:hep-ph/0106012)

[126] Burkardt M 2005 Phys. Rev. D 72094020 (arXiv:hep-ph/0505189)

[127] Polyakov M V and Weiss C 1999 Phys. Rev. D 60114017 (arXiv:hep-ph/9902451)

[128] Mueller D, Robaschik D, Geyer B, Dittes F M and Horejsi J 1994 Fortschr. Phys. 42101 (arXiv:hep-ph/9812448)

[129] Radyushkin A V 1996 Phys. Lett. B 385 333-42 (arXiv:hep-ph/9605431)

[130] Radyushkin A V 1997 Phys. Rev. D 56 5524-57 (arXiv:hep-ph/9704207)

[131] Diehl M 2003 Phys. Rep. 388 41-277 (arXiv:hep-ph/0307382)

[132] Soper D E 1972 Phys. Rev. D 5 1956-62

[133] Burkardt M 2001 (arXiv:hep-ph/0105324)

[134] Pobylitsa P V 2002 Phys. Rev. D 66094002 (arXiv:hep-ph/0204337)

[135] Burkardt M 2003 Int. J. Mod. Phys. A 18 173-208 (arXiv:hep-ph/0207047)

[136] Ageev E S et al (COMPASS) 2007 Nucl. Phys. B 765 31-70 (arXiv:hep-ex/0610068)

[137] Vossen A (COMPASS) 2007 arXiv:0705.2865 [hep-ex]

[138] Orginos K 2006 PoS LAT2006 018

[139] Hagler P 2007 PoS LAT2007 013 (arXiv:0711.0819 [hep-lat])

[140] Brommel D et al (QCDSF/UKQCD) 2007 Eur. Phys. J. C 51 335-345 (arXiv:hep-lat/0608021)

[141] Kaneko T et al (JLQCD) 2007 PoS. LATTICE 2007

[142] Boyle P A, Flynn J M, Juttner A, Sachrajda C T and Zanotti J M 2007 J. High Energy Phys. JHEP05(2007)016 (arXiv:hep-lat/0703005)

[143] Simula S (ETMC) 2007 PoS LAT2007 371 (arXiv:0710.0097 [hep-lat])

[144] Kirch M, Manashov A and Schafer A 2005 Phys. Rev. D 72114006 (arXiv:hep-ph/0509330)

[145] Symanzik K 1983 Nucl. Phys. B 226205

[146] Weisz P and Wohlert R 1984 Nucl. Phys. B 236397

[147] Itoh S, Iwasaki Y and Yoshie T 1986 Phys. Rev. D 331806

[148] Takaishi T 1996 Phys. Rev. D 54 1050-3

[149] Hasenfratz P and Niedermayer F 1994 Nucl. Phys. B 414 785-814 (arXiv:hep-lat/9308004)

[150] Michael C and Urbach C (ETM) 2007 PoS LAT2007 122 (arXiv:0709.4564 [hep-lat])

[151] Dong S J, Liu K F and Williams A G 1998 Prepared for Workshop on Future Directions in Quark Nuclear Physics (Adelaide, Australia, 10-20 March 1998)

[152] Mathur N and Dong S J (Kentucky Field Theory) 2001 Nucl. Phys. Proc. Suppl. 94 311-14 (arXiv:hep-lat/0011015)

[153] Leinweber D B et al 2005 Phys. Rev. Lett. 94212001 (arXiv:hep-lat/0406002)

[154] Leinweber D B et al 2006 Phys. Rev. Lett. 97022001 (arXiv:hep-lat/0601025)

[155] Armstrong D S et al (G0) 2005 Phys. Rev. Lett. 95092001 (arXiv:nucl-ex/0506021)

[156] Aniol K A et al (HAPPEX) 2006 Phys. Rev. Lett. 96022003 (arXiv:nucl-ex/0506010)

[157] Kaplan D B 1992 Phys. Lett. B 288 342-7 (arXiv:hep-lat/9206013)
[158] Shamir Y 1993 Nucl. Phys. B 406 90-106 (arXiv:hep-lat/9303005)

[159] Neuberger H 1998 Phys. Lett. B 417 141-4 (arXiv:hep-lat/9707022)

[160] Edwards R G et al (LHPC) 2006 Phys. Rev. Lett. 96052001 (arXiv:hep-lat/0510062)

[161] Dolgov D et al (LHPC) 2002 Phys. Rev. D 66034506 (arXiv:hep-lat/0201021)

[162] Ohta S and Orginos K (RBCK) 2005 Nucl. Phys. Proc. Suppl. 140 396-8 (arXiv:hep-lat/0411008)

[163] Khan A A et al 2005 Nucl. Phys. Proc. Suppl. 140 408-10 (arXiv:hep-lat/0409161)

[164] Gockeler M et al (QCDSF) 2005 Phys. Lett. B 627 113-23 (arXiv:hep-lat/0507001)

[165] Hagler P et al (LHPC) 2008 Phys. Rev. D 77094502 (arXiv:0705.4295 [hep-lat])

[166] Brömmel D et al (UKQCD) 2007 PoS LAT2007 158 (arXiv:0710.1534 [hep-lat])

[167] Thomas A W 2008 Phys. Rev. Lett. 101102003 (arXiv:0803.2775)

[168] Gockeler M et al (QCDSF) 2007 Phys. Rev. Lett. 98222001 (arXiv:hep-lat/0612032)

[169] Luscher M 1986 Commun. Math. Phys. 104177

[170] Luscher M 1986 Commun. Math. Phys. 105 153-88

[171] Luscher M 1991 Nucl. Phys. B 354 531-78

[172] Burkardt M and Hannafious B 2008 Phys. Lett. B 658 130-7 (arXiv:0705.1573 [hep-ph])

[173] Belitsky A V and Mueller D 1998 Phys. Lett. B 417 129-40 (arXiv:hep-ph/9709379)

[174] Ji X and Osborne J 1998 Phys. Rev. D 58094018 (arXiv:hep-ph/9801260)

[175] Mankiewicz L et al 1998 Phys. Lett. B 425 186-92 (arXiv:hep-ph/9712251)

[176] Anikin I V, Pire B and Teryaev O V 2000 Phys. Rev. D 62071501 (arXiv:hep-ph/0003203)

[177] Radyushkin A V and Weiss C 2001 Phys. Rev. D 63114012 (arXiv:hep-ph/0010296)

[178] Belitsky A V, Mueller D and Kirchner A 2002 Nucl. Phys. B 629 323-92 (arXiv:hep-ph/0112108)

[179] Mergell P, Meissner U G and Drechsel D 1996 Nucl. Phys. A 596 367-96 (arXiv:hep-ph/9506375)

[180] Diehl M, T G, Pire B and Ralston J 1997 Phys. Lett. B 411 193-202 (arXiv:hep-ph/9706344)

[181] Belitsky A V and Mueller D 2002 Nucl. Phys. A 711 118-126 (arXiv:hep-ph/0206306)

[182] Belitsky A V, Mueller D, Niedermeier L and Schafer A 2001 Nucl. Phys. B 593 289-310 (arXiv:hep-ph/0004059)

[183] Brodsky S et al 1972 Phys. Rev D 6172

[184] Kroll P, Schurmann M and Guichon P A M 1996 Nucl. Phys. A 598 435-61 (arXiv:hep-ph/9507298)

[185] Teryaev O V 2005 arXiv:hep-ph/0510031

[186] Kumericki K, Mueller D and Passek-Kumericki K 2008 Nucl. Phys. B 794 244-323 (arXiv:hep-ph/0703179)

[187] Diehl M and Ivanov D Y 2007 Eur. Phys. J. C 52 919-32 (arXiv:0707.0351 [hep-ph])

[188] Guidal M and Vanderhaeghen M 2003 Phys. Rev. Lett. 90012001 (arXiv:hep-ph/0208275)

[189] Belitsky A V and Mueller D 2003 Phys. Rev. Lett. 90022001 (arXiv:hep-ph/0210313)

[190] Collins J C, Frankfurt L and Strikman M 1997 Phys. Rev. D 56 2982-3006 (arXiv:hep-ph/9611433)

[191] Diehl M and Kugler W 2007 Eur. Phys. J. C 52 933-66 (arXiv:0708.1121 [hep-ph])

[192] Boffi S and Pasquini B 2007 Riv. Nuovo Cimento 30387 (arXiv:0711.2625 [hep-ph])

[193] Diehl M, Feldmann T, Jakob R and Kroll P 2005 Eur. Phys. J. C 39 1-39 (arXiv:hep-ph/0408173)

[194] Guidal M, Polyakov M V, Radyushkin A V and 
Vanderhaeghen M 2005 Phys. Rev. D 72054013 (arXiv:hep-ph/0410251)

[195] Gribov V N 1973 arXiv:hep-ph/0006158

[196] Burkardt M 2004 Phys. Lett. B 595 245-9 (arXiv:hep-ph/0401159)

[197] Negele J W et al 2004 Nucl. Phys. Proc. Suppl. 128 170-8 (arXiv:hep-lat/0404005)

[198] Vanderhaeghen M, Guichon P A M and Guidal M 1999 Phys. Rev. D 60094017 (arXiv:hep-ph/9905372)

[199] Petrov V Y et al 1998 Phys. Rev. D 57 4325-33 (arXiv:hep-ph/9710270)

[200] Musatov I V and Radyushkin A V 2000 Phys. Rev. D 61074027 (arXiv:hep-ph/9905376)

[201] Polyakov M V and Shuvaev A G 2002 arXiv:hep-ph/0207153

[202] Kumericki K, Muller D and Passek-Kumericki K 2009 Eur. Phys. J. C 58 193-215 (arXiv:0805.0152 [hep-ph])

[203] Mankiewicz L, Piller G and Radyushkin A 1999 Eur. Phys. J. C 10 307-12 (arXiv:hep-ph/9812467)

[204] Penttinen M, Polyakov M V and Goeke K 2000 Phys. Rev. D 62014024 (arXiv:hep-ph/9909489)

[205] Diakonov D, Petrov V Y and Pobylitsa P V 1988 Nucl. Phys. B 306809

[206] De Alfaro V, Fubini S, Furlan G and Rossetti C 1973 Current in Hadron Physics (Amsterdam: North-Holland) p 571

[207] Polyakov M V 1999 Nucl. Phys. B 555231 (arXiv:hep-ph/9809483)

[208] Diehl M 2008 private communication

[209] Mueller D and Schafer A 2006 Nucl. Phys. B 739 1-59 (arXiv:hep-ph/0509204)

[210] Passek-Kumericki K and Peters G 2008 Phys. Rev. D 78033009 (arXiv:0805.1758 [hep-ph])

[211] Guzey V and Polyakov M V 2006 Eur. Phys. J. C 46 151-6 (arXiv:hep-ph/0507183)

[212] Guzey V and Teckentrup T 2009 Phys. Rev. D 79017501 (arXiv:0810.3899 [hep-ph])

[213] Polyakov M V and Vanderhaeghen M 2008 arXiv:0803.1271 [hep-ph]

[214] Kumericki K and Mueller D 2009 arXiv:0904.0458

[215] Aaron F D et al (H1) 2008 Phys. Lett. B 659 796-806(arXiv:0709.4114 [hep-ex])

[216] Chekanov S et al (ZEUS) 2003 Phys. Lett. B 573 46-62 (arXiv:hep-ex/0305028)

[217] Aktas A et al (H1) 2005 Eur. Phys. J. C 44 1-11 (arXiv:hep-ex/0505061)

[218] Favart L and Machado M V T 2003 Eur. Phys. J. C 29 365-71 (arXiv:hep-ph/0302079)

[219] Favart L and Machado M V T 2004 Eur. Phys. J. C 34 429-33 (arXiv:hep-ph/0402018)

[220] Donnachie A and Dosch H G 2001 Phys. Lett. B 502 74-8 (arXiv:hep-ph/0010227)

[221] Frankfurt L, Strikman M and Weiss C 2005 Ann. Rev. Nucl. Part. Sci. 55 403-65 (arXiv:hep-ph/0507286)

[222] Thomas A W and Weise W 2001 The Structure of the Nucleon (Berlin: Wiley-VCH) p 389

[223] Kumericki K, Mueller D, Passek-Kumericki K and Schafer A 2007 Phys. Lett. B 648 186-94 (arXiv:hep-ph/0605237)
[224] Vogt A, Moch S and Vermaseren J A M 2004 Nucl. Phys. B 691 129-81 (arXiv:hep-ph/0404111)

[225] Aid S et al (H1) 1996 Nucl. Phys. B 470 3-40 (arXiv:hep-ex/9603004)

[226] Munoz Camacho C et al (Jefferson Lab Hall A) 2006 Phys. Rev. Lett. 97262002 (arXiv:nucl-ex/0607029)

[227] Airapetian A et al (HERMES) 2001 Phys. Rev. Lett. 87182001 (arXiv:hep-ex/0106068)

[228] Stepanyan S et al (CLAS) 2001 Phys. Rev. Lett. 87182002 (arXiv:hep-ex/0107043)

[229] Girod F X et al (CLAS) 2008 Phys. Rev. Lett. 100 162002(arXiv:0711.4805 [hep-ex])

[230] Laget J M 2007 Phys. Rev. C 76052201 (arXiv:0708.1250 [hep-ph])

[231] Guidal M 2008 Nucl. Phys. Proc. Suppl. $184234-8$ (arXiv:0803.1592 [hep-ph])

[232] Airapetian A et al (HERMES) 2007 Phys. Rev. D 75011103 (arXiv:hep-ex/0605108)

[233] Airapetian A et al (HERMES) 2008 J. High Energy Phys. JHEP06(2008)066 arXiv:0802.2499 [hep-ex]

[234] Chen S et al (CLAS) 2006 Phys. Rev. Lett. 97072002 (arXiv:hep-ex/0605012)

[235] Kopytin M 2007 Proc. of Quark Nuclear Physics (QNP) 2006 ed A Dobado F J L E and Vento V (Eur. Phys. J. A 31) pp 575-7

[236] Diehl M and Sapeta S 2005 Eur. Phys. J. C 41 515-33 (arXiv:hep-ph/0503023)

[237] Ellinghaus F, Nowak W D, Vinnikov A V and Ye Z 2006 Eur. Phys. J. C 46 729-39 (arXiv:hep-ph/0506264)

[238] Mazouz M et al (Jefferson Lab Hall A) 2007 Phys. Rev. Lett. 99242501 (arXiv:0709.0450 [nucl-ex])

[239] Gockeler M et al 2007 Eur. Phys. J. A 32 445-50 (arXiv:hep-lat/0609001)

[240] Kroll P 2007 arXiv:0710.2771 [hep-ph]

[241] Jaffe R 2008 private communication

[242] Jaffe R L 2001 Phil. Trans. R. Soc. Lond. A 359 391-404 (arXiv:hep-ph/0008038)

[243] Jaffe R L and Manohar A 1990 Nucl. Phys. B 337 509-46

[244] Harindranath A and Kundu R 1999 Phys. Rev. D 59116013 (arXiv:hep-ph/9802406)

[245] Hagler P and Schafer A 1998 Phys. Lett. B 430 179-85 (arXiv:hep-ph/9802362)

[246] Bashinsky S and Jaffe R L 1998 Nucl. Phys. B 536 303-17 (arXiv:hep-ph/9804397)

[247] Brodsky S J 2004 arXiv:hep-ph/0412101

[248] Chen X S, Lu X F, Sun W M, Wang F and Goldman T 2007 arXiv:0709.3649

[249] Chen X S, Lu X F, Sun W M, Wang F and Goldman T 2007 arXiv:0709.1284

[250] Deshpande A, Milner R, Venugopalan R and Vogelsang W 2005 Ann. Rev. Nucl. Part. Sci. 55 165-228 (arXiv:hep-ph/0506148)

[251] Thomas A W 2007 Proc. of 11th Int. Conf. on Meson-Nucleon Physics and the Structure of the Nucleon (MENU 2007) (Julich, Germany, 10-14 September 2007) (eConf C070910,124) 